\title{
Mind your clocks! The role of the biological clock in human metabolic health
}

Citation for published version (APA):

Hansen, J. (2017). Mind your clocks! The role of the biological clock in human metabolic health. [Doctoral Thesis, Maastricht University]. Maastricht University. https://doi.org/10.26481/dis.20171115jh

Document status and date:

Published: 01/01/2017

DOI:

10.26481/dis.20171115jh

Document Version:

Publisher's PDF, also known as Version of record

\section{Please check the document version of this publication:}

- A submitted manuscript is the version of the article upon submission and before peer-review. There can be important differences between the submitted version and the official published version of record.

People interested in the research are advised to contact the author for the final version of the publication, or visit the DOI to the publisher's website.

- The final author version and the galley proof are versions of the publication after peer review.

- The final published version features the final layout of the paper including the volume, issue and page numbers.

Link to publication

\footnotetext{
General rights rights.

- You may freely distribute the URL identifying the publication in the public portal. please follow below link for the End User Agreement:

www.umlib.nl/taverne-license

Take down policy

If you believe that this document breaches copyright please contact us at:

repository@maastrichtuniversity.nl

providing details and we will investigate your claim.
}

Copyright and moral rights for the publications made accessible in the public portal are retained by the authors and/or other copyright owners and it is a condition of accessing publications that users recognise and abide by the legal requirements associated with these

- Users may download and print one copy of any publication from the public portal for the purpose of private study or research.

- You may not further distribute the material or use it for any profit-making activity or commercial gain

If the publication is distributed under the terms of Article $25 \mathrm{fa}$ of the Dutch Copyright Act, indicated by the "Taverne" license above, 


\section{MIND YOUR CLOCKS!}

\section{THE ROLE OF THE BIOLOGICAL CLOCK IN HUMAN METABOLIC HEALTH}

Jan Hansen 


\section{$\mathscr{0}$ NUTRIM}

The research presented in this thesis was performed within NUTRIM School of Nutrition and Translational Research in Metabolism.

Jan Hansen ${ }^{\circledR}$, Maastricht 2017

Cover design: Jan Hansen

Layout: Jan Hansen

Printed by: Gildeprint - Enschede

ISBN: 978-94-6233-795-4 


\section{MIND YOUR CLOCKS!}

\section{THE ROLE OF THE BIOLOGICAL CLOCK IN HUMAN METABOLIC HEALTH}

\section{DISSERTATION}

to obtain the degree of doctor at Maastricht University on the authority of the Rector Magnificus, Prof. Dr. Rianne M Letschert, in accordance with the decision of the Board of Deans, to be defended in public on

Wednesday $15^{\text {th }}$ of November 2017 at 16 hours.

by

\section{Jan Hansen}

Born in Düsseldorf, Germany, on $7^{\text {th }}$ January 1986 


\section{Supervising Committee}

\section{Supervisors}

Prof. Dr. P Schrauwen

Prof. Dr. MKC Hesselink

\section{Assessment committee}

Prof. Dr. B Smeets (chair)

Prof. Dr. K Esser (University of Florida, USA)

Prof. Dr. A Kalsbeek (Academic Medical Center, UvA, Amsterdam)

Prof. Dr. E Mariman

Dr. RCJ Langen 


\section{Table of Content}

CHAPTER 1: $\quad$ General introduction and outline.

CHAPTER 2: Can human muscle sense time?

A model for the molecular clock in health and disease.

CHAPTER 3: Synchronized human skeletal myotubes of lean, obese and type 2 diabetic patients maintain circadian oscillation of clock genes.

CHAPTER 4: Characterization of temporal mitochondrial respiration and protein content in synchronized human primary myotubes of lean, obese and T2D donors.

CHAPTER 5: Demonstration of a day-night rhythm in human skeletal muscle oxidative capacity.

CHAPTER 6: Circadian misalignment induces insulin resistance and affects the skeletal muscle molecular clock in humans.

CHAPTER 7: General discussion and future perspectives.

\section{APPENDIX}

Summary 134

Zusammenfassung

Valorization

Acknowledgements

List of publications

About the Author 



\section{CHAPTER 1}

General introduction and outline 


\section{General Introduction:}

The growing increase in the prevalence of obesity and type 2 diabetes mellitus (T2D) imposes an important global health issue, as this is associated with high morbidity and mortality and causes a major burden on health care costs. Indeed, the major determinants of obesity and T2D are high caloric intake and reduced physical activity, but these factors cannot entirely explain the increasing prevalence of these clinical manifestations. Hence it has been speculated, that this development could be attributed to drastic lifestyle changes in response to the industrialization of our modern society, which may not be restricted to changes in diet and physical activity [1].

With this in mind, it is important to realize that about $15-20 \%$ of the working population is involved in shift work or regularly travels across several time zones [2]. However, disengaging from our natural day-night rhythm is not limited to occupational environment, but rather has become an indispensable part of our society. In fact, in Central Europe over $70 \%$ of a surveyed population suffers from social jetlag [3] - a condition at which one suffers from a jetlag imposed by social aspects, such as working in shift or partying through the night. Similar to travelling time-zones, social jetlag occurs by a misalignment of the behavioral cycle (food, sleep, activity) with the internal biological clock. To date, it is not fully understood how disturbances of the biological clock promote the risk to develop obesity and T2D; yet epidemiological studies demonstrate compelling evidence for an association of shift work [4], late meal timing [5], late chronotype [6] and social jetlag [7] with increased risk for obesity and T2D.

\section{The biological clock}

Our normal day-night rhythms in biological processes are orchestrated by the central biological clock. Interestingly, circadian rhythms are also found in single cells and in small multicellular organisms. Depending on the organism, these circadian rhythms can be imposed by environmental cues such as oscillation in light intensity and wavelength as well as ambient temperature. The term circadian stems from the Latin "circa" (about) and "dies" (day). However, rhythms can also originate from rhythmicity in behavioral cues - such as sleep-wake, and fast-fed phases. The alignment of the environmental and behavioral cues can offer advantages in survival, which are specific to the organism. Hence, humans have evolved adhering to the solar cycle. This alignment to a circadian rhythm is enabled by a 
mechanism known as the biological clock. According to our current understanding, which mainly originates from rodent studies, the biological clock comprises of a central clock situated in the suprachiasmatic nucleus (SCN) of the hypothalamus. This central clock senses time by accounting for important internal and external time cues, such as ambient light, activity and food consumption. The central clock is thought to be composed of a neuronal network to produce robust neuronal and humoral outputs to orchestrate the circadian organization of behavioral, physiological, neuronal and cellular processes. In order to synchronize the entire body, peripheral cells are considered to further sustain molecular clocks that enable cells to keep track of time individually. To result in temporal homeostasis, each peripheral molecular clock comprises of an autonomous but adaptable mechanism to resonate in synchrony with the body. In CHAPTER 3 of this thesis, a detailed explanation of the molecular clock is given. In general, the molecular clock is suspected to govern fundamental tissue specific functions to anticipate when a tissue should excel in its physiological function.

Cellular Time Keeping

Cellular Function

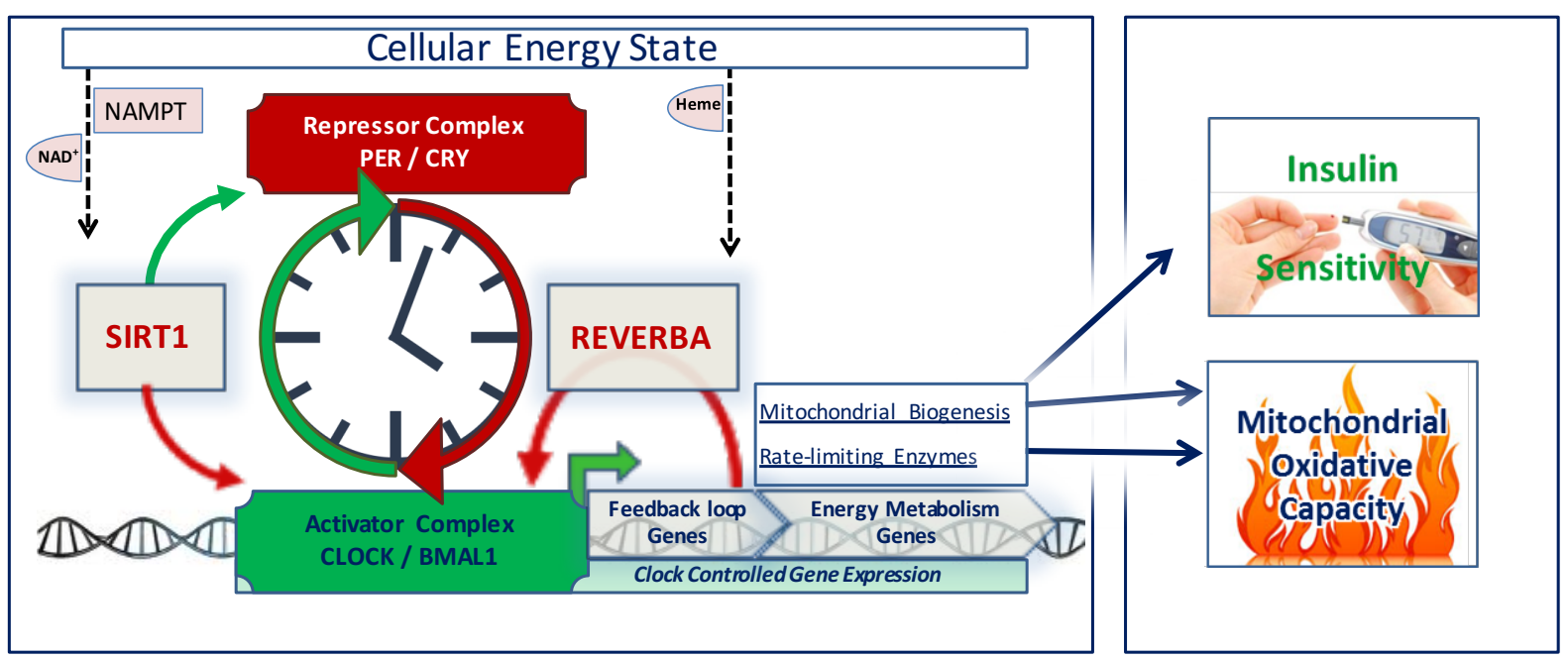

Figure 1: BMAL1 and CLOCK heterodimerize to initiate clock controlled gene expression (CCGE). Subsequently, PER and CRY are expressed forming a repressive complex to suppress CCGE. An interlocked feedback loop is formed by cyclic REVERBA expression. In addition, the core molecular clock is further regulated by activity of SIRT1 and REVERBA, driven by the cellular energy state and nutrient availability. The molecular clock can drive gene expression to orchestrate tissue specific function to the time in need. 


\section{The biological clock and metabolism}

The research on the impact of the biological clock is complex, as environmental, behavioral and circadian rhythms, are indispensably intertwined. In general, circadian rhythms are characterized as intrinsic rhythms that occur independently from environmental and behavioral time cues. In fact, identifying circadian rhythms requires the absolute isolation or constant presence of time cues such as light or food. For this purpose, constant routine protocols have been employed in humans, using constant light and hourly ingestion of food. An alternative to investigate the origin of a rhythm is to isolate cells of a certain tissue to study tissue specific circadian rhythmicity in vitro. In CHAPTER 2,3 and $\mathbf{4}$ this methodology was set-up and employed using human primary myotubes isolated from human skeletal muscle. While these studies reveal the origin of a rhythm, the physiological impact remains to be measured in a normal lifestyle situation to conclude its relevance to metabolic health. Hence, it is of great importance to first characterize human whole-body temporal energy homeostasis in a normal day-night rhythm, with aligned circadian, behavioral and environmental rhythms, as was studied in CHAPTER 5. On this basis, to further the question can be addressed how a disturbed biological clock contributes to derangements in energy metabolism, and hence to determine the impact of circadian misalignment on insulin sensitivity and mitochondrial metabolism as was studied in CHAPTER 6.

\section{Outline:}

The purpose of the research outlined in this thesis was to investigate a possible link between the disruption of the human biological clock and derangements in human energy metabolism, and to determine to which extent this contributes to the etiology of obesity and type 2 diabetes mellitus.

To investigate this, we employed two different strategies: A cellular model (CHAPTER 2, 3, 4) and human intervention studies (CHAPTER 5, 6). CHAPTER 2 briefly summarizes the methodological development of a cellular model that was used to explore circadian rhythms of human skeletal muscle. Subsequently in CHAPTER 3, this model is used to investigate intrinsic circadian rhythms in skeletal muscle cultures of donors with a wide range in metabolic health; from athletes to lean sedentary men, and obese but otherwise healthy men to obese type 2 diabetic patients. In these groups, we also characterize group 
differences in rhythmic expression of genes encoding metabolic sensors and rate-limiting enzymes. In CHAPTER 4, we explore whether such differences translate to changes in temporal mitochondrial respiration, and whether human mitochondrial function is intrinsically rhythmic.

The second part of this thesis sheds light on rhythmic energy homeostasis of healthy human volunteers. In CHAPTER 5, we describe an exploratory study with young male volunteers to characterize the 24-hour variation in whole body energy metabolism and skeletal muscle mitochondrial function as well as molecular clock gene expression. Against this background, in CHAPTER 6, we investigated the effect of a 12-hour misalignment of the biological clock with the behavioral cycle on insulin sensitivity, mitochondrial metabolism and energy metabolism in healthy volunteers.

Finally, in CHAPTER 7, we discuss the findings of this thesis in the light of the current literature and speculate about possible aspects to improve future prevention and treatment strategies for obesity and type 2 diabetes. 


\section{References}

[1] Qian, J. and F.A. Scheer, 2016. Circadian System and Glucose Metabolism: Implications for Physiology and Disease. Trends Endocrinol Metab 27(5):282-293.

[2] Haus, E. and M. Smolensky, 2006. Biological clocks and shift work: circadian dysregulation and potential long-term effects. Cancer Causes Control 17(4):489-500.

[3] Roenneberg, T., K.V. Allebrandt, M. Merrow, and C. Vetter, 2012. Social jetlag and obesity. Curr Biol 22(10):939-943.

[4] Pan, A., E.S. Schernhammer, Q. Sun, and F.B. Hu, 2011. Rotating night shift work and risk of type 2 diabetes: two prospective cohort studies in women. PLoS Med 8(12):e1001141.

[5] Garaulet, M., P. Gomez-Abellan, J.J. Alburquerque-Bejar, Y.C. Lee, J.M. Ordovas, and F.A. Scheer, 2013. Timing of food intake predicts weight loss effectiveness. Int J Obes (Lond) 37(4):604-611.

[6] Yu, J.H., C.H. Yun, J.H. Ahn, S. Suh, H.J. Cho, S.K. Lee, et al., 2015. Evening chronotype is associated with metabolic disorders and body composition in middle-aged adults. J Clin Endocrinol Metab 100(4):1494-1502.

[7] Parsons, M.J., T.E. Moffitt, A.M. Gregory, S. Goldman-Mellor, P.M. Nolan, R. Poulton, et al., 2015. Social jetlag, obesity and metabolic disorder: investigation in a cohort study. Int J Obes (Lond) 39(5):842-848. 


\section{CHAPTER 2}

Can human muscle sense time?

A model for the molecular clock in health and disease.

Authors:

Jan Hansen, Esther Moonen-Kornips, Matthijs KC Hesselink, Patrick Schrauwen 


\section{Abstract}

The biological clock has been proposed to be an intricate part in governing cellular and tissue specific processes. Cells sustain a molecular clock to regulate gene expression temporally. With its central role in cellular and whole-body homeostasis, a disturbed molecular clock has been linked to several clinical manifestations ranging from cancer to cardiovascular and metabolic diseases. However, identifying which processes are truly orchestrated by the molecular clock and which are induced by environmental and behavioral rhythms is technically very demanding in living organisms. Hence, here we report the validation of a cellular model, to enable studying circadian rhythms of human skeletal muscle.

Biopsy derived human primary myocytes were differentiated to myotubes. Serum shock was employed to synchronize human myotubes to determine molecular clock gene expression over a time course of 72 hours. Cultures of different donors showed similar phase with pronounced amplitude in core molecular clock gene expression. For this model, we obtained human myocytes from donors ranging in metabolic health; from healthy lean athletes and sedentary lean to obese and type 2 diabetic patients. To be able to relate the molecular characteristics of the molecular clock to metabolic parameters, the donor's in vivo metabolic phenotype of the corresponding cultures was characterized.

In conclusion, we demonstrate a cellular model that could be used to investigate intrinsic circadian rhythms of skeletal muscle independent of donor (patho)physiology. Further, this model bears the potential to be used for testing optimal drug timing and efficacy, to improve and develop pharmacological strategies. 


\section{Introduction}

Human skeletal muscle is a central organ in energy metabolism. As it accounts for $80 \%$ of the postprandial glucose-uptake, it has great influence on glucose homeostasis [1]. Given its size and its central role in facilitating human locomotion, it also accounts for the greatest share of total energy expenditure in humans, both in activity and at rest [2]. With its influence on plasma glucose clearance and energy expenditure, skeletal muscle metabolism is important in the etiology of obesity and type 2 diabetes. In type 2 diabetes, glucose uptake in skeletal muscle is reduced due to insulin resistance. Interestingly, at the same time impairments in mitochondrial function have been reported in patients with type 2 diabetes. The underlying mechanisms that lead to such metabolic derangements are still under investigation. In this thesis, the possible link of circadian disturbances on a cellular and whole-body level are discussed. This chapter recapitulates the methodological development of a cell model that enables investigating the nature of circadian rhythms in human skeletal muscle in primary cell cultures of donors with different metabolic health status.

Before starting this thesis project, data from animal studies have suggested that in mammalian organs autonomous molecular clocks govern tissue specific gene expression, explained in detail in CHAPTER 3. Thus, the molecular clock was subjected to temporally orchestrate tissue specific function in human skeletal muscle. For instance, insulin sensitivity was reported to be regulated by intrinsic circadian rhythms as shown in cultured rat primary myotubes [3]. Further, it could be shown that disrupting the molecular clock by knockout of BMAL1, led to insulin resistance in mice [4]. Together, this suggested that the insulin action depends on an intact molecular clock. Additionally, it could be shown that the molecular clock governs expression of tissue specific targets, as shown for MyoD, which is a master regulator in myogenesis [5]. Upon molecular clock disruption, using Clock ${ }^{\Delta 19}$ and $\mathrm{Bmal}^{-/-}$ mutant mice, skeletal muscle was functionally impaired. For instance, mutant mice had reduced muscle force together with a substantial reduction in mitochondrial respiration and volume, when compared to wildtype mice [5]. The muscle-specific ablation of BMAL1 in another mouse model also showed impaired muscle force [6]. Intriguingly, this model also demonstrates that disruption of the muscle molecular clock resulted in impaired insulin 
stimulated glucose uptake [6]. Also REVERBA, a molecular clock gene of the repressing loop, was shown to have a central role in the regulation of mitochondrial function [7]. Whilst overexpression of REVERBA improved mitochondrial function, deficiency in REVERBA resulted in reduction of mitochondrial content and oxidative function [7]. Noteworthy, together the findings of Andrews et al. [5], Woldt et. al. [7] and Ramsey et al [8] revealed that there is a close link between molecular clock genes and the AMPK-SIRT1-PGC1 cascade. This cascade is of particular interest as it plays a crucial role in the regulation of (oxidative) metabolism in skeletal muscle and has been associated with promoting metabolic health [9], as shown in aging, calorie restriction, food compound supplementation and exercise.

Hence, we hypothesized that skeletal muscle sustains a molecular clock with intrinsic circadian rhythms. In order to address this, we here describe a selection of cell experiments that lead to creation of a novel cellular model to investigate human skeletal muscle circadian rhythms, isolated from donor physiology. We were particularly interested in setting-up a model using human primary myotubes, to allow the translation of results obtained in mouse studies towards potential human relevance.

\section{Methods and Results:}

\section{Study design and subject characteristics}

We included primary myotubes from subjects that had previously participated in our studies $[10,11]$. Human primary myotubes were cultured from the following four groups: 1) young endurance trained lean athletes (trained lean - TL), 2) age-matched young, lean sedentary controls (untrained lean - UL), and 3) middle-aged healthy, obese subjects (OB) and 4) BMIand age-matched type 2 diabetes patients (T2D) (Table 1). ). Inclusion criteria for endurance trained athletes were performing endurance exercise (long-distance running, cycling, swimming) at least three times a week for the past 2 years and a VO2max $>55 \mathrm{ml} / \mathrm{min} / \mathrm{kg}$; all other subjects performed less than 1 h of exercise per week for the past 2 years. Type 2 diabetic patients were diagnosed at least 1 year prior to the study, were non-insulin dependent, were well-controlled ( $\mathrm{HbA} 1 \mathrm{c}<64 \mathrm{mmol} / \mathrm{mol})$, and had no diabetes-related comorbidities. All type 2 diabetes patients had been treated with metformin or metformin + 
sulfonylureas for at least 6 months. All studies were approved by the local Medical Ethical Committee of Maastricht University according to the declaration of Helsinki principles. All participants gave their written informed consent.

\section{Primary muscle cell culture}

As described in detail $[10,11]$, biopsies were obtained after an overnight fast between 8 to 9 AM.

Primary skeletal muscle cell cultures were established by isolating and growing needle biopsy derived satellite cells in media supplemented with 16\% FBS (Gibco, Thermo

Fisher Scientific, Waltham, USA) at $37{ }^{\circ} \mathrm{C}$ and 5\% CO2. After 7-10 days, mono-nucleated satellite cells migrated and adhered to the culture dish. The remainder of the biopsy was removed. An additional proliferation phase of 14 days was used to augment cell number. When the donor culture reached $70-80 \%$ confluence, cells were trypsinized (Gibco, Thermo Fisher Scientific, Waltham, USA) and stored in liquid nitrogen. This option, to have repeatedly access to donor cultures, is one of the advantages of this model. For instance, several donor cultures can be compared in the same experimental setup and used again for another experimental approach at a later stage, ensuring low passage numbers. For each donor biopsy, initial satellite cultures yielded approximately 10-16 aliquots containing $5 \times 10^{5}$ cells.

For each experiment, differentiation to multi-nucleated skeletal myotubes was induced by seeding mono-nucleated satellite cells confluently in differentiation media $\left(5 \times 10^{4}\right.$ cells $/ 10$ $\mathrm{cm}^{2}$; passage < 5); Minimum Essential Medium $\alpha$ (Gibco, Thermo Fisher Scientific, Waltham, USA) supplemented with $2 \%$ FBS (Gibco, Thermo Fisher Scientific, Waltham, USA) and fetuin (Sigma-Aldrich, St. Louis, USA). Every other day differentiation media was changed.

As previously reported by us [10] and others, cultured myotubes retain their in vivo muscle phenotype in terms of insulin sensitivity [12-14] and mitochondrial lipid metabolism [10, 14, 15].

\section{In-vitro synchronization of primary myotubes}

Individual cells are proposed to run on individual clocks. If multiple cells are being studied for intervention or donor effects on clock related processes, differences may fade, if the cells run asynchronously. Thus, prior to the actual experiment, cells needed to be 
synchronized. Various stimuli have been demonstrated to be able to synchronize cells. Because the ultimate goal of this model has been to compare cultures of donors with different health status, we aimed to use a synchronization agent that was not directly regulating or interfering with mitochondrial oxidative capacity or insulin sensitivity. Therefore, we refrained from using compounds such as dexamethasone, as it is a highly potent glucocorticoid analog, which was reported to induce insulin resistance in muscle [16, 17]. Hence, we employed the widely-used serum shock as a synchronization stimulus. Serum shock consisted of a two-hour incubation with fresh differentiation media, supplemented with $50 \%$ horse serum (Gibco, Thermo Fisher Scientific, Waltham, USA). After serum shock, cultures were washed and supplemented with fresh differentiation media.

\section{Synchronization Protocols}

We first attempted to investigate, whether human skeletal muscle myotubes indeed express core clock genes in an oscillatory manner. Hence, cultures were harvested 4-hourly for 48 hours, starting from 16 hours after serum shock. In this first pilot, we used aliquots of myocytes harvested from an obese donor pool, comprising of six different donor cultures. As hypothesized, we found oscillatory gene expression of the core molecular clock gene BMAL1 with a first peak around 36 hours after serum shock (figure 1). Additional analysis of repressor genes PER and CRY revealed opposed gene expression rhythms (data not shown), as described for the mammalian clock [18]. A practical disadvantage of this experimental design is that the continuous sampling for several consecutive days is very demanding on the experimentator in terms of sleep and daily schedule (figure 1A). Thus, in a second pilot we implemented two parallel timelines, in order to be able to measure molecular clock gene oscillation for three consecutive days. One timeline consisted of a batch of serum shocks started in the morning, whilst another batch was initiated 12 hours later, as outlined in figure $1 \mathrm{~A}$. With this approach, harvesting cultures could be scheduled more flexible, and therefore favouring the sleep and daily schedule of the experimentator.

When comparing the BMAL1 expression profiles of the linear- with the parallel timeline model, in both approaches an oscillatory pattern occurs with peaks and troughs at similar times (figure 1B). Given the practical advantages for the experimentator and the longer time period that could be observed, we pursued the investigation with the parallel timeline model. 
A
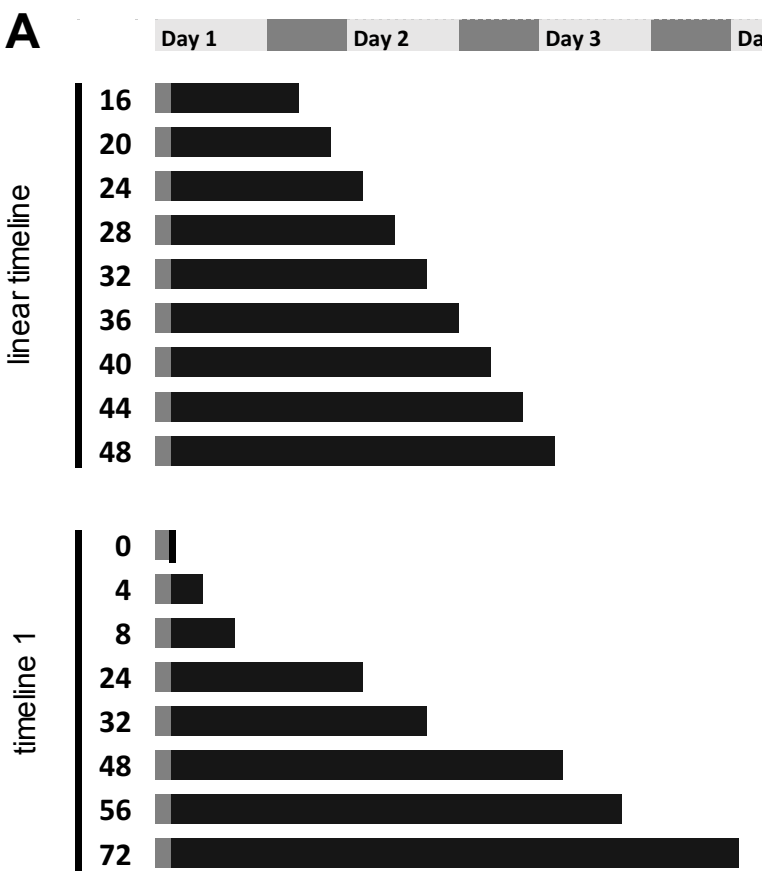

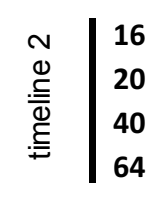

Day 1
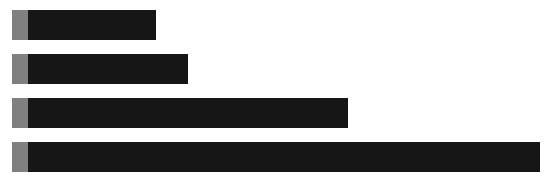

Day 2

Day 3

Day 4

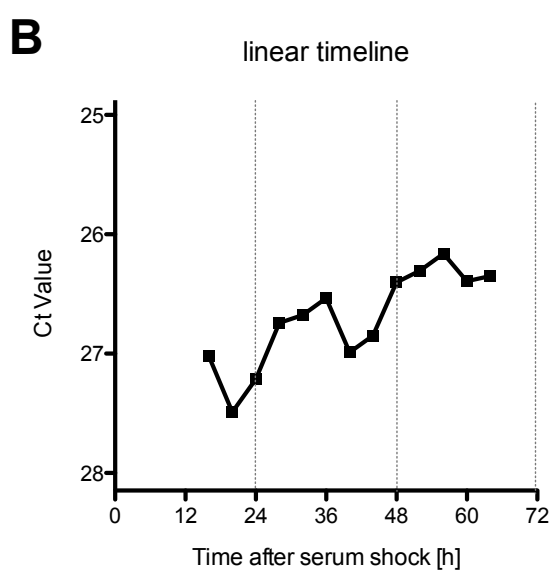

parallel timeline

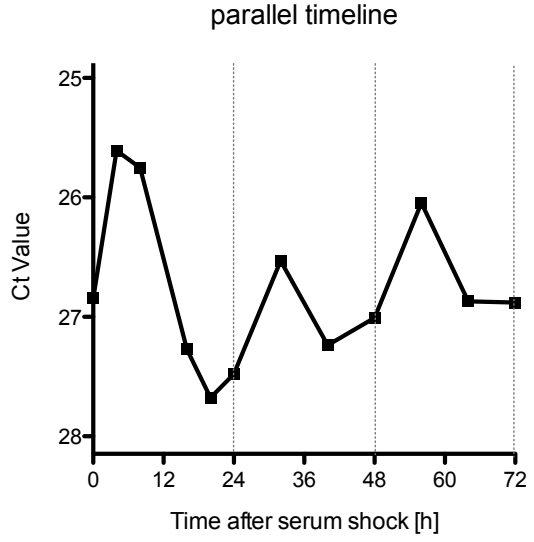

Figure 1: A) time schedule of serum shock (grey blocks). Numbers indicate time after serum shock at harvest (black bars). Upper model, linear timeline consists of one time point for serum shock and consequent 4-hourly harvest. Below, parallel timeline consists of two serum shock batches at $8 A M$ and $8 P M$, allowing a simpler scheduling for the experimentator. $B)$ BMAL1 expression in synchronized human primary myotubes of obese donor cultures of the two different methodolical approaches. Scale is reversed; lower Ct numbers represent higher expression.

\section{Gene expression and correction}

On-dish TRIzol lysis (Qiagen, Hilden, Germany) was applied to ensure high yield and RNA integrity. RNA was further purified using the RNeasy kit from Qiagen (Hilden, Germany). The quality and yield of RNA was assessed using a NanoDrop spectrophotometer (Thermo Fisher Scientific, Waltham, USA). The high-capacity RNA-to-cDNA kit from Applied Biosystems (Foster City, USA) was used for transcription of $0.5 \mu \mathrm{g}$ RNA to cDNA. Transcript abundance 
was determined using a 7900HT Fast Real-Time PCR System (RT-pPCR) (Applied Biosystems, Waltham, USA).

Transcript abundance is usually normalized for housekeeping genes (HKG) in RT-QPCR, but housekeeping genes can sometimes be affected by the experimental condition itself. Hence, using a single HKG comes with the risk to induce variation and possibly alter effect size. As up to $20 \%$ of protein coding transcripts are subject to circadian control [19], first we selected HKG to test for circadian variation: HPRT1, ACTB, RPLP0, GAPDH, 18S, RPL26, GUSB and CYPB. In figure 2A, in pooled cells of T2D HPRT1 expression fluctuated over time $(\Delta C t>1)$, as also seen for other common HKG (data not shown). We found that RPL26, GUSB and CYPB were the three HKG with the least temporal variation (figure 2), in each of the the four donor groups, shown in detail in supplemental figure 1 of CHAPTER 3. To further minimize the influence of the variability of each housekeeping gene on target gene normalization, a geometric mean of the three housekeeping genes was used for each time point. This approach resulted in a geometric mean with strong robustness over time, as shown exemplarily for T2D myotubes in figure 2A. Therefore, the geometric mean was used as the internal reference for the comparative gene expression analysis for the remainder of the study.

A

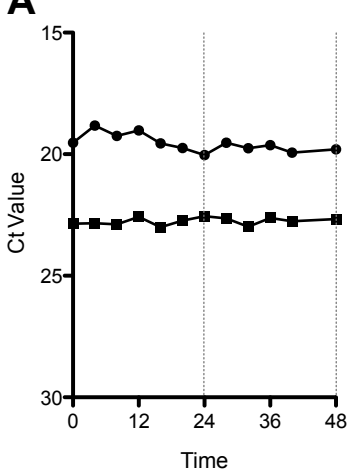

B

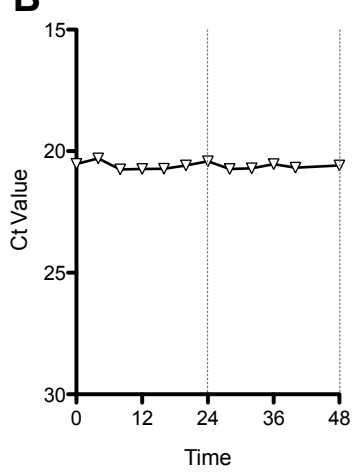

C

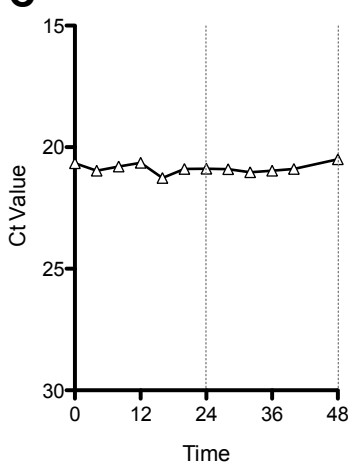

D

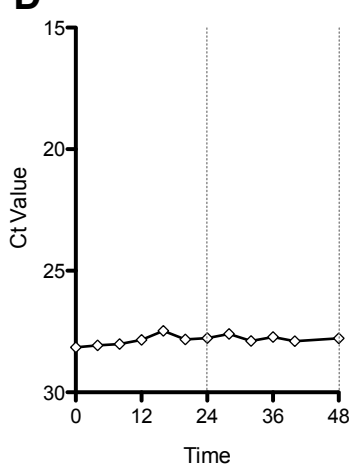

Figure 2: Housekeeping gene expression of T2D myotube culture. A) Comparison of expression of commonly used HKG HPRT1 against the geometric mean, comprised of B) RPL26, C) GUSB, D) CYPB expression. Values, represent quantification of 4-hourly harvested cultures $n=1$. Black squares depict GEOMEAN, black circles depict HPRT1 expression. Scale is reversed; lower Ct numbers represent higher expression. 
As demonstrated in figure 1 , clock genes were expressed in a time dependent manner. In that experiment, we used pools of individual cell cultures. Although pooled cells reduce the number of test samples vastly, the use of a culture pool does not allow correlational analysis of the corresponding donor in vivo data. Furthermore, the use of a pool bears the risk, of unequal proliferation and differentiation between the donors and hence the pool may be dominated by a single donor, rather than representing the mean response of the donors included. Hence, we aimed to investigate if a pool of cells is representative to the rhythms of the individual cultures. To this end, cultures of three individuals were grown and seeded individually parallel to the pool of the same three donors. As depicted in figure 3 , cultures of pool and lean individuals share similar peak and trough times for the activator gene BMAL1 and repressor loop genes PER2 and REVERBA. Close observation of all three transcript profiles reveals that the pool resembles the profiles of lean donor 1 and 2 more accurately than that of donor 3. Indeed, for BMAL1, PER2 and REVERB two-way ANOVA, revealed significant differences for time and donor interaction $(p<0.01)$. This indicates that although all cultures appear to have the same circadian phase, individual gene expression rhythms vary between donors. Therefore, we preferred investigating the molecular clock genes in individual cultures. In this way we achieved a more accurate representation of the intimate link between donor physiology and its molecular clock.
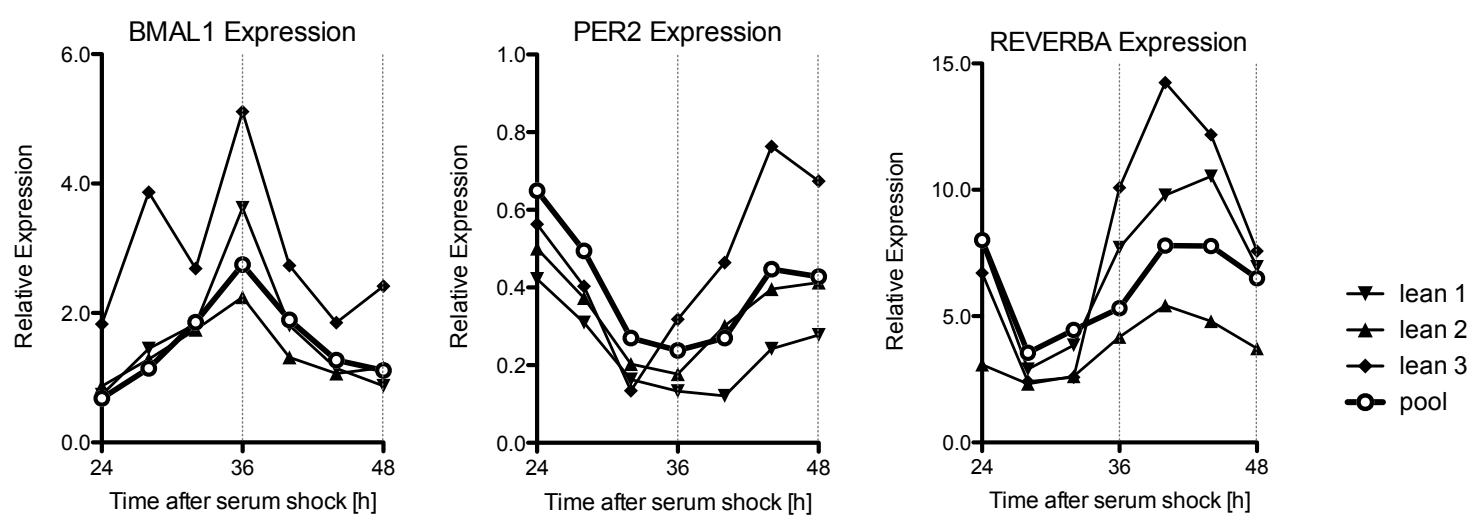

Figure 3: Molecular clock gene expression of activator A), and repressor feedback loops B) and $C$ ). Values are relative expression of single timepoints of three different donors and a culture of all three donors pooled immediately before differentiation. 
A

Geomean HKG

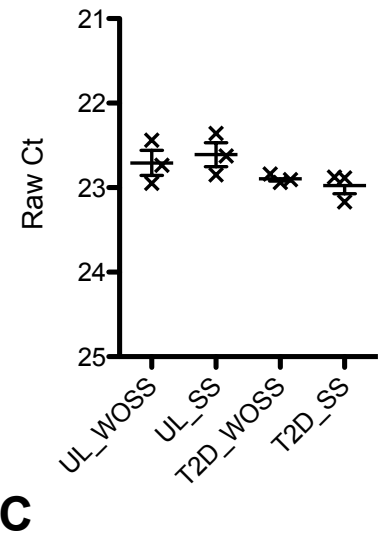

REVERBA

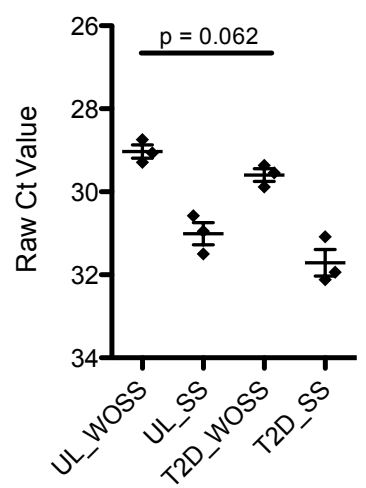

D

NAMPT

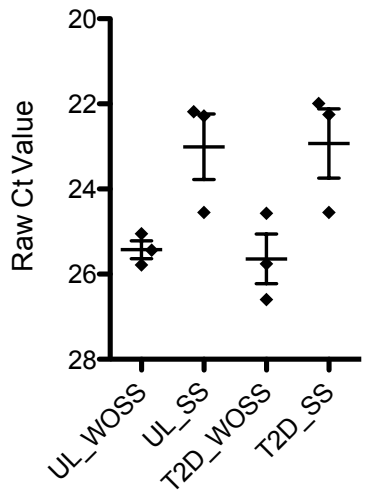

B

CLOCK

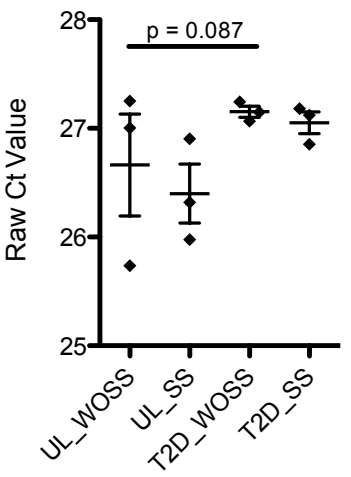

PER2 Expression

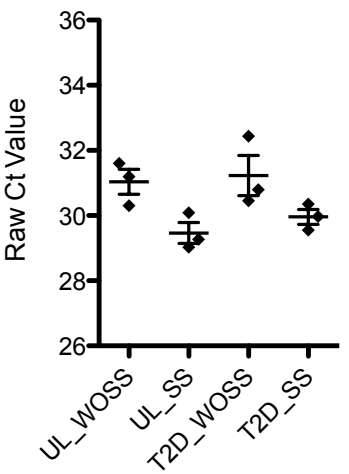

SIRT1

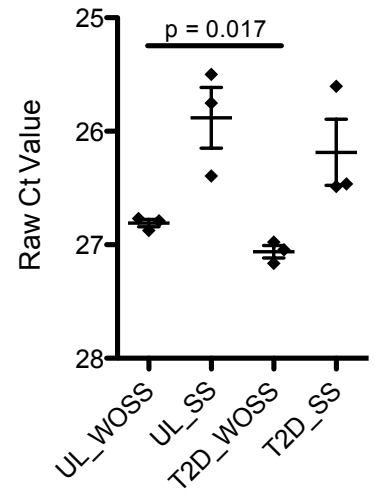

BMAL1

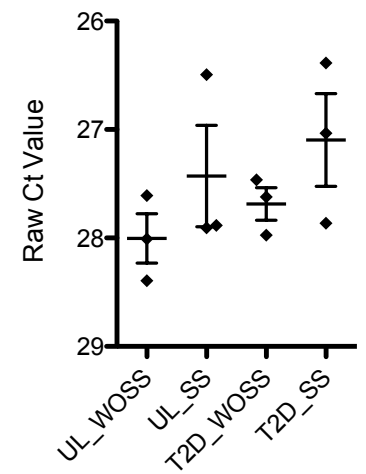

CRY1 Expression

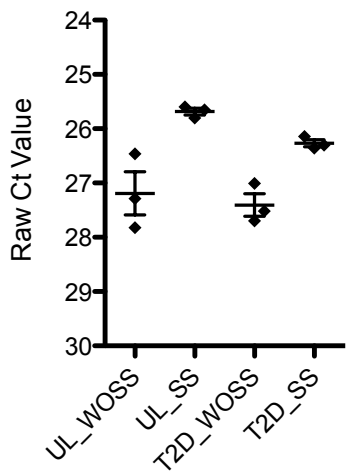

PPARd

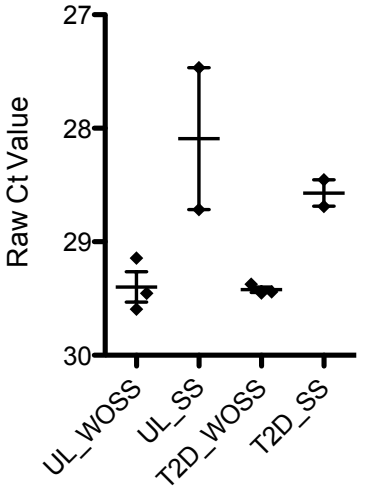

Figure 4: Panels depict gene expression either without serum shock (WOSS) or after serum shock (SS). A) Geometric mean of RPL26, GUSB and CYPB, B) core molecular clock genes of activator complex and C) repressor loop, D) metabolic markers. Data is represented in Ct cycle of threshold. Scale is reversed; lower Ct numbers represent higher expression. Values are mean with SEM, $n=3$ 
As mentioned before, cultured myotubes were reported to retain their muscle phenotype in terms of insulin sensitivity [12-14] and mitochondrial lipid metabolism [10, 14, 15]. We therefore aimed to investigate if cell cultures originating from different donor groups display differences in molecular clock gene expression. However, since in our circadian studies, muscle cell cultures were exposed to serum shock, we also aimed to examine if serum shock affects muscle cell gene expression. Therefore, we measured gene expression in fully differentiated primary myotubes of UL and T2D donors first without synchronization (without serum shock - WOSS). We found similar housekeeping gene expression between groups (figure $4 \mathrm{~A}, \mathrm{p}>0.05$ ), allowing the comparison of raw $\mathrm{Ct}$ (cycle of threshold) between groups. Students t-test revealed that before serum shock SIRT1 expression was significantly lower in T2D cultures compared to UL $(p=0.017$ ) (Figure 4D). Further we observed trends for lower expression of REVERBA ( $p=0.062$, figure $4 C$ ) and elevated CLOCK expression $(p=0.087$, Figure 4B) in T2D compared to UL. Lower expression of SIRT1 has been associated to overweight and T2D [20]. Previously, in animal and human experiments, lower expression of SIRT1 and REVERBA has been associated to attenuated mitochondrial function [7, 9, 21]. REVERBA also had been shown to be relevant for muscle lipid metabolism in animal studies $[7,22]$. A characteristic of skeletal muscle in T2D is excess storage of neutral lipids. To examine if serum shock had effects on muscle gene expression, we then compared gene expression without serum shock with gene expression at 0 baseline after serum shock (SS). After serum shock, none of the measured genes were statistically different expressed between groups anymore ( $p>0.05)$, suggesting that differences between donor groups could be lost by serum shock treatment.

Therefore, we tested whether mitochondrial function was different between donor cultures after serum shock, as previously published in supplemental figure of CHAPTER 3. Here, we compared the respiration rates of human primary myotube cultures of TL with $U L$ and $O B$ with T2D to estimate mitochondrial function. Indeed, we found reduced mitochondrial respiration in cultures of T2D donors when compared to healthy controls (OB). This indicated that also after serum shock donor phenotype characteristics were retained. 


\section{Conclusion}

Taken together, here we demonstrated a selection of experiments that were a directive to setting up a cellular model, which could be used to investigate the make-over of the molecular clock in health and metabolic disease. We show that human primary myotubes can be used to study circadian rhythmicity in gene expression, but did not reveal differences between donor groups after serum shock. For future experiments, options for changing the culture milieu to a more pathophysiologic environment could be used to reveal how the molecular clock of healthy and T2D donors changes under hyperinsulinemic and hyperglycemic conditions. Furthermore, this model bears the potential to be used for testing drug timing and temporal drug efficacy, to improve and develop pharmacological strategies. 


\section{References}

[1] Defronzo, R.A., D. Simonson, E. Ferrannini, and E. Barrett, 1981. Insulin resistance: a universal finding in diabetic states. Bull Schweiz Akad Med Wiss:223-238.

[2] Zurlo, F., K. Larson, C. Bogardus, and E. Ravussin, 1990. Skeletal muscle metabolism is a major determinant of resting energy expenditure. J Clin Invest 86(5):1423-1427.

[3] Feneberg, R. and B. Lemmer, 2004. Circadian rhythm of glucose uptake in cultures of skeletal muscle cells and adipocytes in Wistar-Kyoto, Wistar, Goto-Kakizaki, and spontaneously hypertensive rats. Chronobiol Int 21(4-5):521-538.

[4] Shi, S.Q., T.S. Ansari, O.P. McGuinness, D.H. Wasserman, and C.H. Johnson, 2013. Circadian disruption leads to insulin resistance and obesity. Curr Biol 23(5):372-381.

[5] Andrews, J.L., X. Zhang, J.J. McCarthy, E.L. McDearmon, T.A. Hornberger, B. Russell, et al., 2010. CLOCK and BMAL1 regulate MyoD and are necessary for maintenance of skeletal muscle phenotype and function. Proc Natl Acad Sci U S A 107(44):1909019095.

[6] Dyar, K.A., S. Ciciliot, L.E. Wright, R.S. Bienso, G.M. Tagliazucchi, V.R. Patel, et al., 2014. Muscle insulin sensitivity and glucose metabolism are controlled by the intrinsic muscle clock. Mol Metab 3(1):29-41.

[7] Woldt, E., Y. Sebti, L.A. Solt, C. Duhem, S. Lancel, J. Eeckhoute, et al., 2013. Rev-erbalpha modulates skeletal muscle oxidative capacity by regulating mitochondrial biogenesis and autophagy. Nat Med 19(8):1039-1046.

[8] Ramsey, K.M., J. Yoshino, C.S. Brace, D. Abrassart, Y. Kobayashi, B. Marcheva, et al., 2009. Circadian clock feedback cycle through NAMPT-mediated NAD+ biosynthesis. Science 324(5927):651-654.

[9] Canto, C. and J. Auwerx, 2009. PGC-1alpha, SIRT1 and AMPK, an energy sensing network that controls energy expenditure. Curr Opin Lipidol 20(2):98-105.

[10] Sparks, L.M., M. Bosma, B. Brouwers, T. van de Weijer, L. Bilet, G. Schaart, et al., 2014. Reduced incorporation of fatty acids into triacylglycerol in myotubes from obese individuals with type 2 diabetes. Diabetes 63(5):1583-1593.

[11] Vosselman, M.J., J. Hoeks, B. Brans, H. Pallubinsky, E.B. Nascimento, A.A. van der Lans, et al., 2015. Low brown adipose tissue activity in endurance-trained compared with lean sedentary men. Int J Obes (Lond) 39(12):1696-1702.

[12] Green, C.J., M. Pedersen, B.K. Pedersen, and C. Scheele, 2011. Elevated NF-kappaB activation is conserved in human myocytes cultured from obese type 2 diabetic patients and attenuated by AMP-activated protein kinase. Diabetes 60(11):28102819.

[13] Bourlier, V., C. Saint-Laurent, K. Louche, P.M. Badin, C. Thalamas, I. de Glisezinski, et al., 2013. Enhanced glucose metabolism is preserved in cultured primary myotubes from obese donors in response to exercise training. J Clin Endocrinol Metab 98(9):3739-3747.

[14] Ukropcova, B., M. McNeil, O. Sereda, L. de Jonge, H. Xie, G.A. Bray, et al., 2005. Dynamic changes in fat oxidation in human primary myocytes mirror metabolic characteristics of the donor. J Clin Invest 115(7):1934-1941.

[15] Gaster, M., A.C. Rustan, V. Aas, and H. Beck-Nielsen, 2004. Reduced lipid oxidation in skeletal muscle from type 2 diabetic subjects may be of genetic origin: evidence from cultured myotubes. Diabetes 53(3):542-548. 
[16] Weinstein, S.P., T. Paquin, A. Pritsker, and R.S. Haber, 1995. Glucocorticoid-induced insulin resistance: dexamethasone inhibits the activation of glucose transport in rat skeletal muscle by both insulin- and non-insulin-related stimuli. Diabetes 44(4):441445.

[17] Tappy, L., D. Randin, P. Vollenweider, L. Vollenweider, N. Paquot, U. Scherrer, et al., 1994. Mechanisms of dexamethasone-induced insulin resistance in healthy humans. J Clin Endocrinol Metab 79(4):1063-1069.

[18] Ko, C.H. and J.S. Takahashi, 2006. Molecular components of the mammalian circadian clock. Hum Mol Genet 15 Spec No 2(R271-277.

[19] Reddy, A.B., N.A. Karp, E.S. Maywood, E.A. Sage, M. Deery, J.S. O'Neill, et al., 2006. Circadian orchestration of the hepatic proteome. Curr Biol 16(11):1107-1115.

[20] Song, Y.S., S.K. Lee, Y.J. Jang, H.S. Park, J.H. Kim, Y.J. Lee, et al., 2013. Association between low SIRT1 expression in visceral and subcutaneous adipose tissues and metabolic abnormalities in women with obesity and type 2 diabetes. Diabetes Res Clin Pract 101(3):341-348.

[21] Rutanen, J., N. Yaluri, S. Modi, J. Pihlajamaki, M. Vanttinen, P. Itkonen, et al., 2010. SIRT1 mRNA expression may be associated with energy expenditure and insulin sensitivity. Diabetes 59(4):829-835.

[22] Kumar Jha, P., E. Challet, and A. Kalsbeek, 2015. Circadian rhythms in glucose and lipid metabolism in nocturnal and diurnal mammals. Mol Cell Endocrinol 418 Pt 1(7488. 


\section{CHAPTER 3}

Synchronized human skeletal myotubes of lean, obese and type 2 diabetic patients maintain circadian oscillation of clock genes

Published: Scientific Reports 2016, 6:35047

\section{Authors}

Jan Hansen, Silvie Timmers, Esther Moonen-Kornips, Helene Duez, Bart Staels, Matthijs KC Hesselink, Patrick Schrauwen 


\begin{abstract}
:
Cell and animal studies have demonstrated that circadian rhythm is governed by autonomous rhythmicity of clock genes. Although disturbances in circadian rhythm have been implicated in metabolic disease development, it remains unknown whether muscle circadian rhythm is altered in human models of type 2 diabetes. Here we used human primary myotubes (HPM) to investigate if rhythmicity of clock- and metabolic gene expression is altered in donors with obesity or type 2 diabetes compared to metabolically healthy donors. HPM were obtained from skeletal muscle biopsies of four groups: type 2 diabetic patients and their BMI- and age-matched obese controls and from lean, healthy and young endurance trained athletes and their age-matched sedentary controls. Cultured HPM were synchronized by serum shock and subsequent gene expression was measured for 72 hours. HPM display robust circadian rhythms in clock genes, but REVERBA displayed dampened rhythmicity in type 2 diabetes. Furthermore, rhythmicity in NAMPT and SIRT1 expression was only observed in HPM from trained athletes. Rhythmicity in expression of key-regulators of carbohydrate and lipid metabolism was modest. We demonstrate that in human skeletal muscle REVERBA/B, NAMPT and SIRT1 circadian rhythms are affected in donors of sedentary life style and poor health status.
\end{abstract}




\section{Introduction}

Skeletal muscle plays an important role in maintaining whole body energy and substrate metabolism and is responsible for $\sim 80 \%$ of postprandial glucose uptake in humans [1]. Type 2 diabetes (T2D) is indeed characterized by disturbances in skeletal muscle insulin sensitivity and mitochondrial function $[2,3]$, and the disability of skeletal muscle to adjust substrate oxidation to glucose or fat availability - called metabolic inflexibility - is associated with the development of muscle insulin resistance and type 2 diabetes [4]. These findings not only illustrate the importance of skeletal muscle in modulating blood glucose levels, but also indicate that in order to maintain metabolic health a fast switch between substrates is necessary to cope with the daily nutrient challenges.

To anticipate such metabolic adjustments at the cellular levels, peripheral tissues generate circadian oscillations by an intricate transcriptional-translational feedback loop, known as the molecular clock. This clockwork involves an activating limb, comprising the heterodimer of Brain and muscle ARNT-Like 1 (BMAL1) and circadian locomotor output cycles kaput (CLOCK). This complex promotes gene expression at E-boxes, and controls thereby the expression of period (PER), cryptochrome (CRY) and nuclear receptor subfamily 1 , group $D$, member 1 (REVERBA). The repressing limb comprises REVERBA, REVERBB and the heterodimer of PER with CRY. The timely shifted translocation of the PER/CRY heterodimer into the nucleus inhibits the activating limb. Independently, REVERBA inhibits the expression of BMAL1 $[5,6]$. In addition, the cellular energy status, reflected by nicotinamide adenine dinucleotide $\left(\mathrm{NAD}^{+}\right)$, can activate sirtuin 1 (SIRT1), which in turn represses the active limb by deacetylation of both activating and repressing limb components [7]. The fine-tuning of this mechanism is closely regulated on both limbs by post-transcriptional modifications [7-9]. Apart from keeping time, this clockwork governs the transcription of clock-controlled genes, such as tissue-specific transcription factors of carbohydrate- and lipid metabolism [10-13], but also of genes involved in mitochondrial biogenesis [13, 14]. Notwithstanding, the peripheral molecular clock of skeletal muscle is also sensitive to hypothalamic-independent signals such as fasted-fed cycles and/or physical exercise [10, 15-18].

In recent years, evidence is emerging that disturbances in the circadian clock may lead to metabolic diseases. For example glucose homeostasis was severely disturbed in rodent knock out and deficiency models of BMAL1 [19-22], REVERBA [23], PER [20] and CRY [24]. 
Also, liver- and overall body fat were adversely affected in knockout mice of BMAL1 and REVERBA. Lastly, the muscle-specific inactivation of Bmal1 in mice lead to muscle insulin resistance, altered muscle glucose metabolism and ultimately to substantially elevated muscle and body weight [25].

Also in human studies, clock gene expression is associated with metabolic health, as adipose tissue clock gene expression was shown to correlate with waist circumference [26] and BMI [27]. Furthermore, macronutrient availability has been shown to influence clock gene expression in blood monocytes of healthy subjects [28]. However, whether circadian rhythmicity of clock and metabolic genes in muscle is altered in humans with type 2 diabetes is so far unknown. In order to characterize the autonomous molecular clock of human muscle in relation to type 2 diabetes, a human primary myotube model was used. Human primary myotubes maintain many of the metabolic characteristics of their donor [29-31]. Moreover, it was recently shown that circadian rhythmicity of clock genes is maintained in human primary myotubes derived from healthy donors [32]. Therefore, human primary myotubes can be used to investigate if circadian rhythmicity in metabolic gene expression is affected in type 2 diabetes. Here, we cultured human primary myotubes from donors with a broad range in metabolic health status and insulin sensitivity, i.e. ranging from young healthy endurance trained athletes to type 2 diabetes patients. We aimed to investigate whether gene expression of key metabolic pathways in cultured human primary myotubes displayed circadian rhythmicity and whether this is impacted by the phenotypic characteristics of the donor. 


\section{Results}

\section{Clock genes show circadian rhythmicity in human primary myotubes}

We first determined gene expression profiles of the core molecular clock components to examine circadian rhythmicity. BMAL1 expression showed 24-hour rhythmicity in all four donor groups as detected by JTK_CYCLE (figure 1A, $p<0.01$ ). One-way ANOVA analysis revealed that in all groups gene expression was indeed significantly different between time points $(p<0.05)$, and post-hoc analysis revealed significant differences between peaks at $36 \mathrm{~h}$ and $64 \mathrm{~h}$ after serum shock and a corresponding nadir at $24 \mathrm{~h}, 48 \mathrm{~h}$ and $72 \mathrm{~h}$ after serum shock in all groups $(p<0.05)$. Two-way ANOVA revealed no significant interaction effect for BMAL1 expression, suggesting that the rhythmicity of BMAL1 expression was not different between donor groups $(p>0.05)$.

It has previously been reported, that the heterodimer partner of BMAL1, CLOCK, features minimal circadian variation [33, 34]. Here, we confirm that CLOCK gene expression revealed no circadian rhythmicity in human primary myotubes (figure 1A, JTK_CYCLE p>0.05).

\section{Expression of negative feedback loop genes show circadian rhythmicity}

The components of the repressor complex - PER2 and CRY1 - are known to oscillate in a $12 \mathrm{~h}$ to $16 \mathrm{~h}$ shift to the rhythm of BMAL1. Indeed, PER2 showed circadian rhythmicity for all donor groups (figure 1B, JTK_CYCLE p<0.001), One-way ANOVA analysis revealed that gene expression was indeed significantly different between time points of cells from untrained lean (UL) and sedentary obese $(O B)(p<0.05)$, and post-hoc analysis revealed significant differences in time with peaks at $24 \mathrm{~h}$ and $52 \mathrm{~h}$ and nadirs at $36 \mathrm{~h}$ and $64 \mathrm{~h}$ after serum shock, which is in anti-phase with the expression profile of BMAL1 (figure 1A,B). Although similar rhythmicity was visible in cells of trained lean (TL) and type 2 diabetic patients (T2D), differences in time did just not reach statistical significance $(p=0.13$ and $p=0.12$ in TL and T2D respectively).

Gene expression of CRY1 also showed significant circadian rhythmicity in all groups (figure 1B, JTK_CYCLE $p<0.01)$. The circadian rhythm patterns of PER2 and CRY1 were not significantly different between donor groups, as we observed no interaction effect using two-way ANOVA analysis ( $p>0.05)$. 

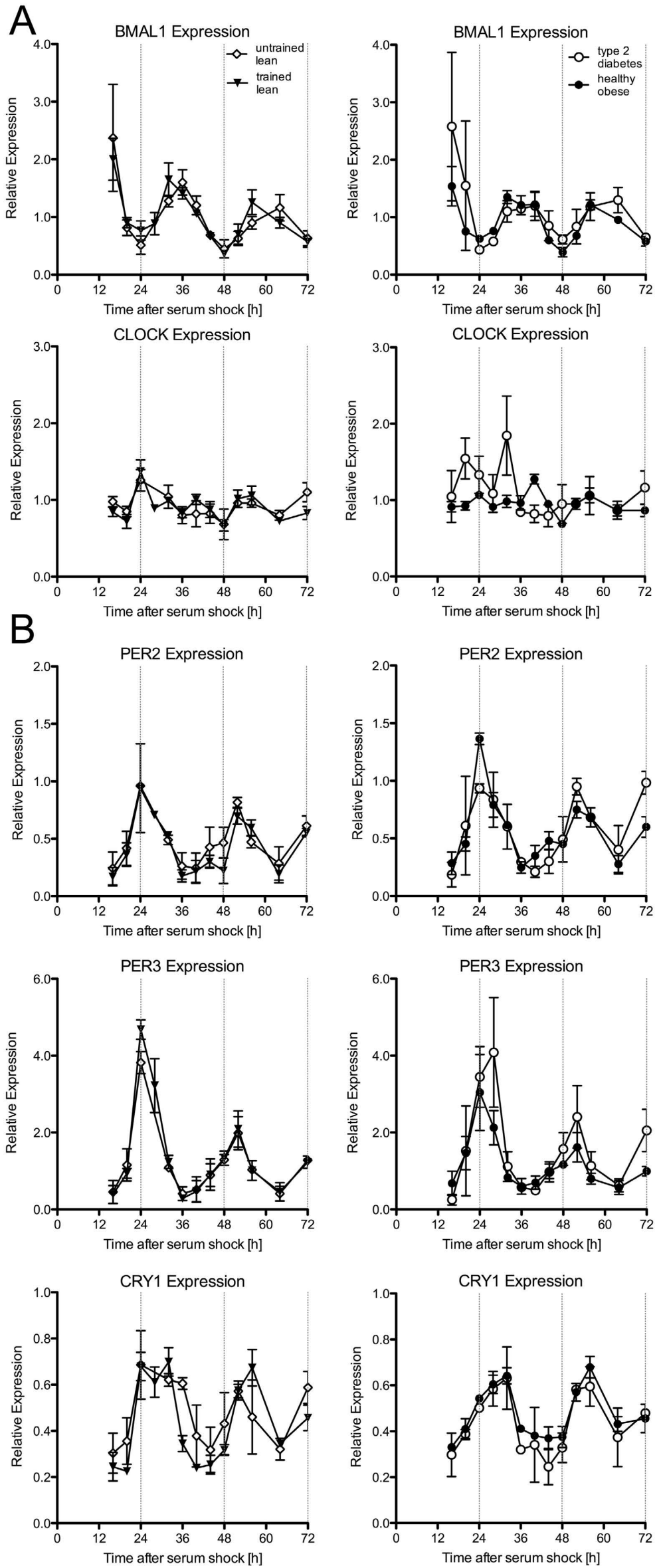
Figure 1: Rhythmic gene expression of core components of the molecular clock.

The relative transcript abundance of the positive $(A)$ and negative limb (B) components of the molecular clock was quantified by RT-PCR and normalized to the corresponding geometric mean of RPL26, CYPB and GUSB. Each value consists of the average of the independent cultures from three different donors. The value of the sample taken immediately after serum shock (Oh) was normalized to 1. Expression profiles of synchronized differentiated primary myotube cultures from trained lean (black triangle), untrained lean (open diamond), obese (black circle) and type 2 diabetes patients (open circle) are shown. Data are mean \pm SEM.

Next, we measured the transcripts of genes that have been reported to be under the transcriptional control of CLOCK and BMAL1, and to be involved in the fine-tuning of the circadian clock. Both for REVERBA and REVERBB, 24-hour circadian rhythmicity was observed in athletes, lean sedentary and obese subjects (figure 2A, JTK_CYCLE p<0.001), but the rhythm was dampened in type 2 diabetes patients (figure 2A, JTK_CYCLE p>0.05). Oneway ANOVA analysis revealed significantly different REVERBA levels between time points in all groups (figure $2 \mathrm{~A}, \mathrm{p}<0.05$ ). Post-hoc analysis revealed that REVERBA peaked at 20 to $24 \mathrm{~h}$ and again at $40 \mathrm{~h}$ to $44 \mathrm{~h}$, with the corresponding nadirs at $32 \mathrm{~h}$ and $52-56 \mathrm{~h}$. For REVERBB, highest expression was determined at $24 \mathrm{~h}$ and at $48 \mathrm{~h}$, and lowest expression at $16 \mathrm{~h}, 36 \mathrm{~h}$ and 56h (figure 2A) in all groups except for type 2 diabetes patients (ANOVA TL, UL, OB: $p<0.001$, T2D: $p=0.473)$.

A circadian rhythm was observed for SIRT1 only in athletes (figure $\mathbf{2 B}, \mathbf{p}<0.05$ ). One-way ANOVA also revealed a statistical significant time effect in athletes only $(p<0.05)$, with highest expression at $24 \mathrm{~h}$ and $52 \mathrm{~h}$, and lowest expression at 36 and $64 \mathrm{~h}$. In concordance with SIRT1 expression, analysis of NAMPT expression also confirmed a circadian rhythm in athletes only (figure $2 \mathrm{~B}, \mathrm{p}<0.001$ ), although one-way ANOVA did not reveal significant differences between the time points. 

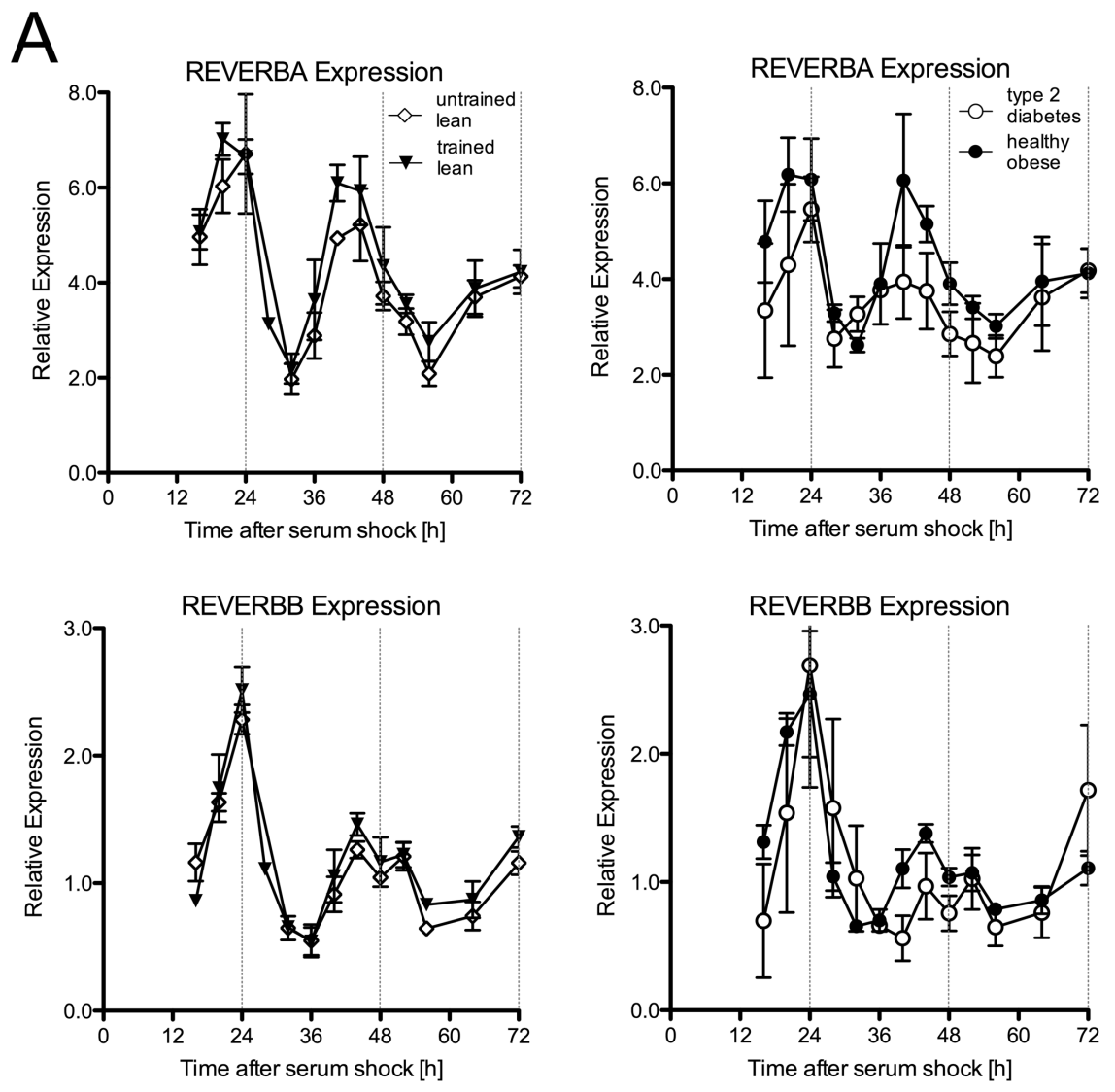

B
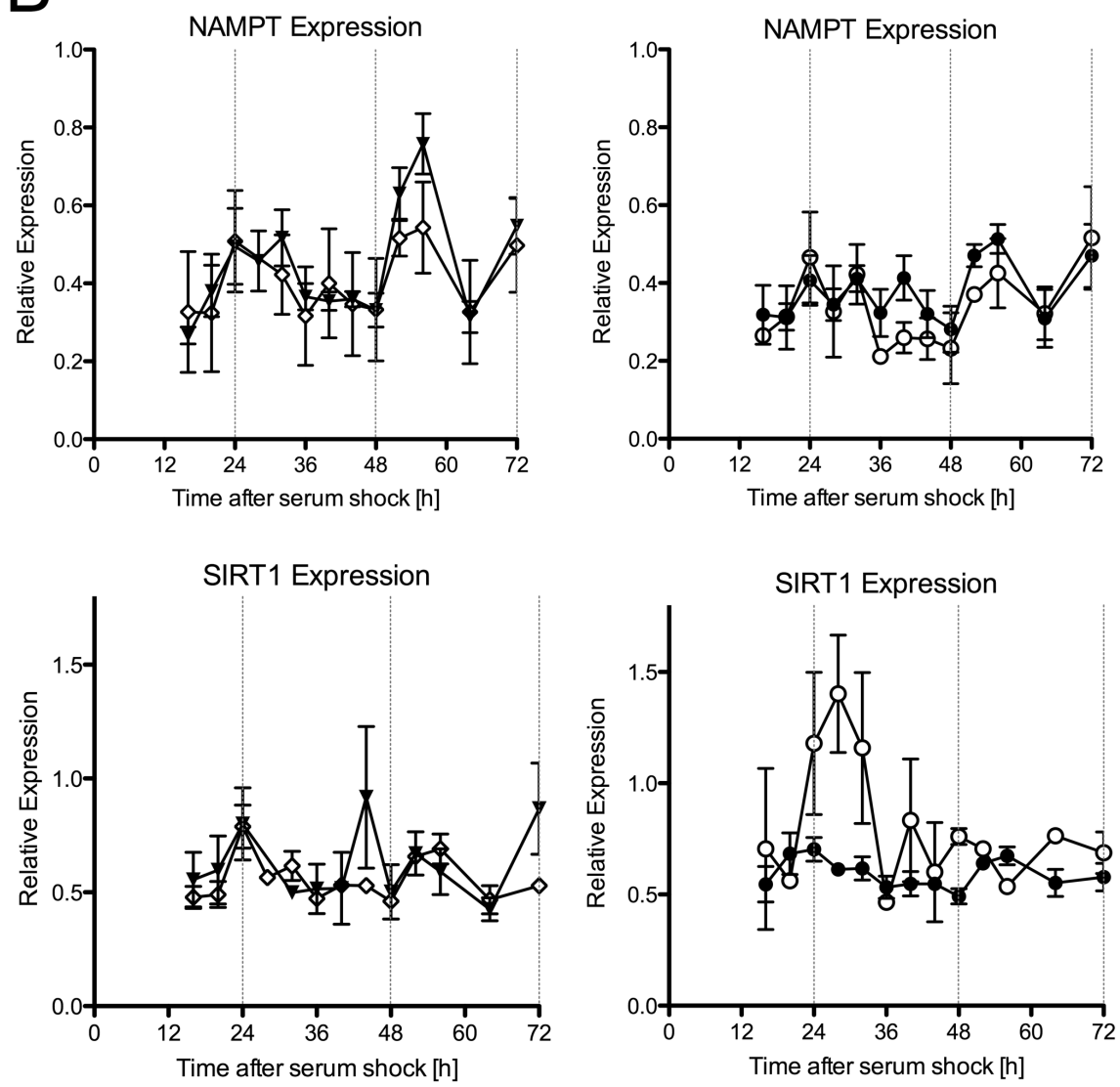
Figure 2: Rhythmic gene expression of molecular clock components.

The relative abundance of transcripts that are under the control of the Ebox domain was quantified by RT-PCR and normalized to the corresponding geometric mean of RPL26, CYPB and GUSB. Each value consists of the average of the independent cultures from three different donors. The value of the sample taken immediately after serum shock (Oh) was normalized to 1. Expression profiles of synchronized differentiated primary myotube cultures from trained lean (black triangle), untrained lean (open diamond), obese (black circle) and type 2 diabetes patients (open circle) are shown. Data are mean \pm SEM.

\section{Modest circadian rhythmicity in expression of genes involved in substrate metabolism}

We next measured transcript abundance of genes that are key regulators in cellular glucose metabolism. Circadian rhythmicity determined by JTK_CYCLE was found in gene expression of glycogen synthase 1 (GYS1) for type 2 diabetes patients only, but not for its inhibitory regulator - the glycogen synthase kinase 3 beta (GSK3b). For hexokinase II (HKII) JTK_CYCLE indicated rhythmicity in sedentary lean and tendencies in obese and type 2 diabetic donors (figure 2, UL $p<0.05 ; O B p=0.07 ; T 2 D p=0.09, T L p=1.00$ ); However, one-way ANOVA revealed no significant differences in HKII gene expression in time in sedentary lean $(p>0.05)$. Also, as can be seen from figure 3 , the pattern of HKIl suggests a linear increase in HKII over time. Finally, we measured insulin receptor substrate 1 (IRS1) - a gene involved in insulin-stimulated glucose uptake - which only revealed a rhythmic pattern using JTK_CYCLE in athletes (supplemental figure 3, JTK_CYCLE $p<0.01$ ).

We next also determined gene expression of several genes in mitochondrial function. Therefore, we measured the mitochondrial adenine nucleotide transporter (ANT) that exchanges ATP and ADP in the mitochondrial matrix. Further we quantified the peroxisome proliferator-activated receptor alpha (PPARa) and delta (PPARd), which both are involved in regulating lipid metabolism and mitochondrial biogenesis. Circadian rhythmicity was found in gene expression of ANT in sedentary lean and for PPARa in athletes (JTK_CYCLE p<0.05), but one-way ANOVA did not reveal significant differences in time for any of the groups (figure 4, $p>0.05$ ). 

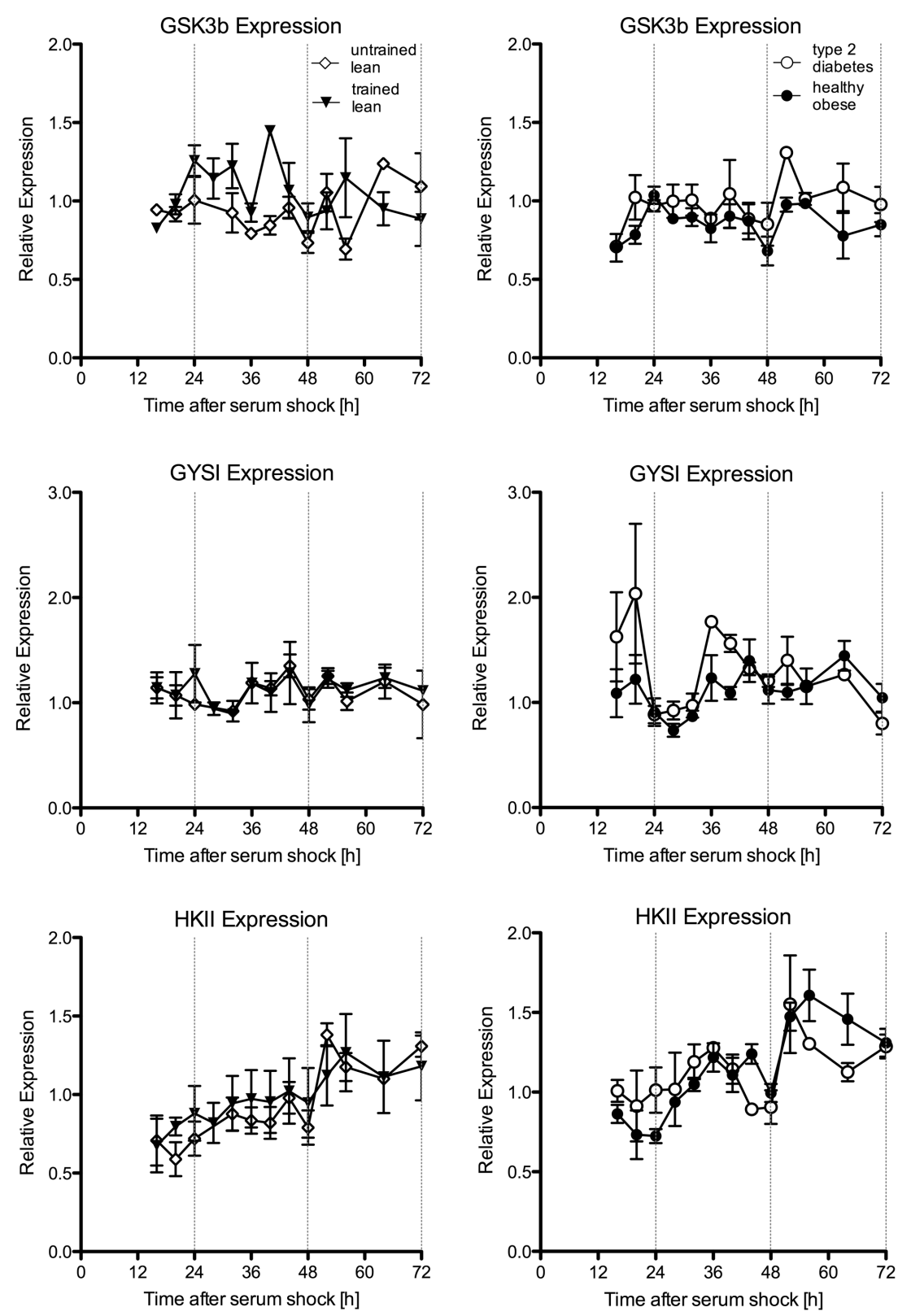

Figure 3: Expression profiles of cellular glucose metabolism genes.

The relative abundance of transcripts that encode proteins of cellular glucose and glycogen metabolism was quantified by RT-PCR and normalized to the corresponding geometric mean of RPL26, CYPB and GUSB. Each value consists of the average of the independent cultures from three different donors. The value of the sample taken immediately after serum shock (Oh) was normalized to 1. Expression profiles of synchronized differentiated primary myotube cultures from trained lean (black triangle), untrained lean (open diamond), obese (black circle) and type 2 diabetes patients (open circle) are shown. Data are mean \pm SEM. 

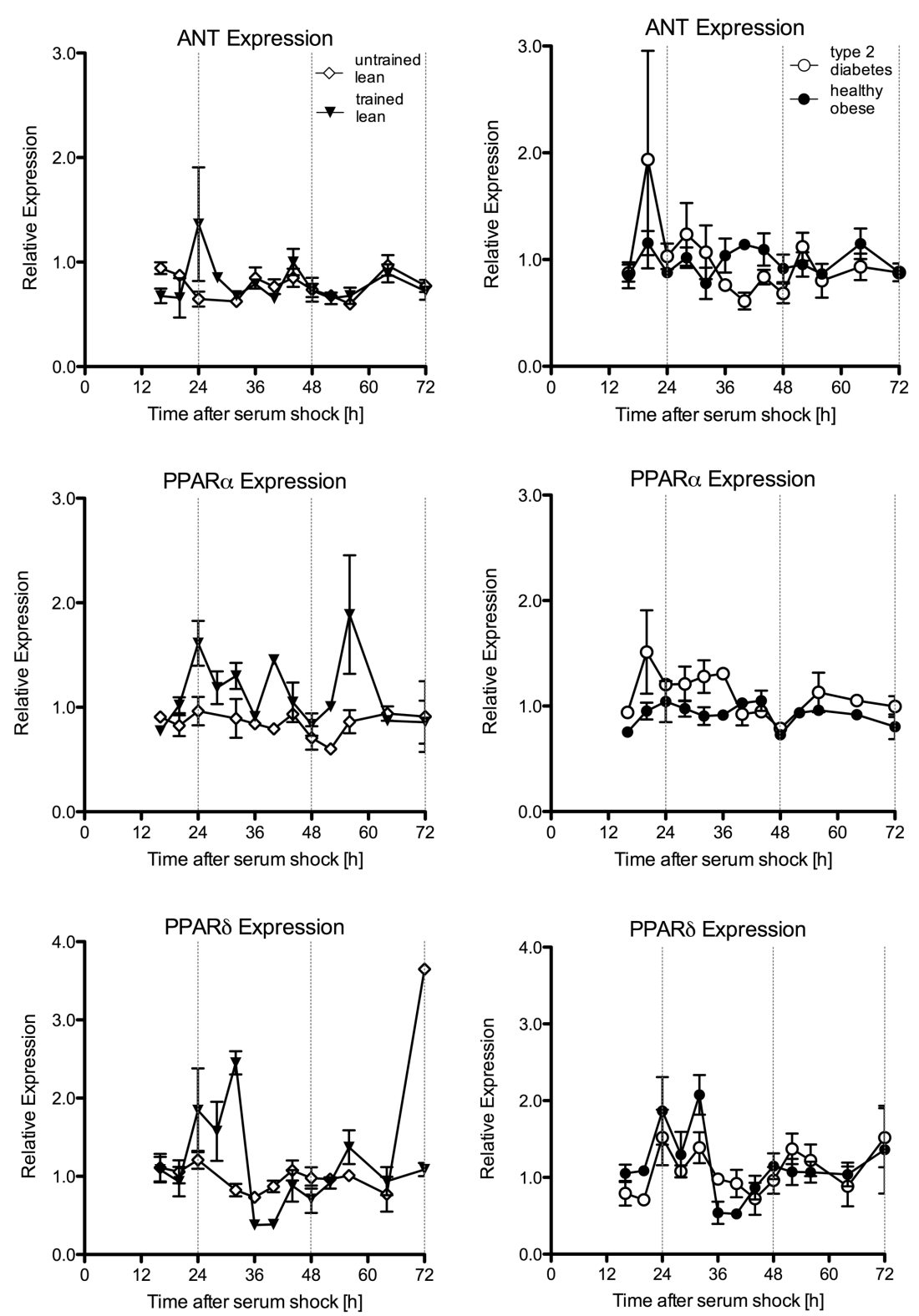

Figure 4: Expression profiles of genes involved in mitochondrial metabolism.

The relative transcript abundance of transcripts that encode proteins involved in mitochondrial function and biogenesis was quantified by RT-PCR and normalized to the corresponding geometric mean of RPL26, CYPB and GUSB. Each value consists of the average of the independent cultures from three different donors. The value of the sample taken immediately after serum shock (Oh) was normalized to 1. Expression profiles of synchronized differentiated primary myotube cultures are plotted as follows: trained lean (black triangle) against untrained lean (open diamond) and obese (black circle) against type 2 diabetics (open circle). Data are mean \pm SEM 
For PPARd, circadian rhythmicity was observed in athletes, obese and T2D using JTK-cycle (JTK_CYCLE $\mathrm{p}<0.05)$, and one-way ANOVA analysis revealed significantly different gene expression levels of PPARd between time points in athletes and obese, $p<0.001$ and $\mathrm{p}<0.009$, respectively. Post-hoc analysis revealed that PPARd was significantly higher at $28 \mathrm{~h}$ vs. $36 \mathrm{~h}$ in TL. Furthermore, we determined gene expression of DGAT1, the rate-limiting enzyme in the conversion of diacylglycerol into triacylglycerol. Circadian rhythm was revealed in lean sedentary subjects only using JTK-CYCLE (supplemental figure 3, p>0.05), but one-way ANOVA did not reveal significant differences in time in lean sedentary subjects (supplemental figure 3).

\section{REVERBA}

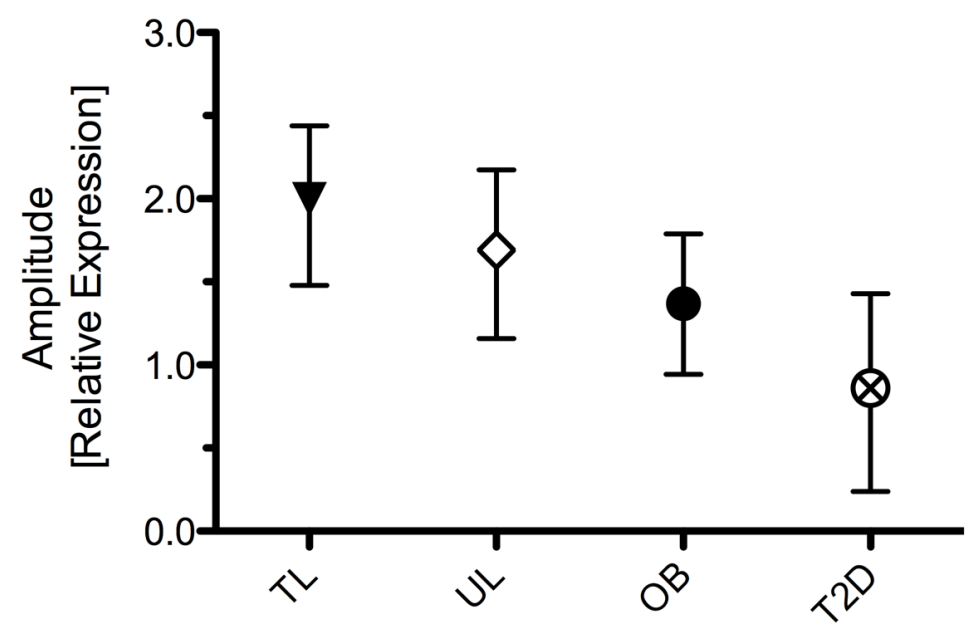

Figure 5: Correlative analysis of circadian rhythmicity with the metabolic phenotype of the donor.

The plot displays the association (Spearman's Rho test for nominal data) between the metabolic phenotype of the donor with the amplitude of the JTK_CYCLE based peak and nadir for REVERBA. Data are relative expression amplitude with $95 \% \mathrm{Cl}$ as error bars. 


\section{Discussion}

Rodent studies have revealed the importance of the CLOCK/BMAL1 component in the regulation of autonomous circadian rhythm in skeletal muscle [14, 25]. Recently, circadian rhythmicity was also shown in human primary myotubes [32], which we confirm here. Our results reveal that circadian rhythmicity for the core clock components was not affected by the metabolic status of the donor. Interestingly however, REVERBA and REVERBB analysis showed no circadian rhythmicity in human primary myotubes derived from type 2 diabetes patients, and rhythmicity for SIRT1 and NAMPT was only observed in myotubes from endurance trained athletes. These findings may suggest that the molecular clock system in human skeletal muscle is affected by metabolic condition and may be involved in the etiology of chronic metabolic disease.

The aim of the current study was to examine if circadian rhythmicity in skeletal muscle is affected by the characteristics of the donors, whom were ranging in metabolic health from endurance trained athletes to metabolically-compromised T2D patients. We find that serum shock is capable of inducing synchronization in human primary myotubes from a skeletal muscle biopsy. In line with the study of Balsalobre et al. [35], also in human muscle cells, oscillation of autonomous clock components persists at least throughout $72 \mathrm{~h}$ after serum shock synchronization. This robust oscillation was reflected by all measured components of the transcriptional-translational feedback loop. Especially, BMAL1 and REVERBA demonstrated the highest amplitudes in rhythmicity. Furthermore, the characteristic phase delay of REVERBA/B, PER and CRY to BMAL1, previously observed in human biopsies [34, 36] and human primary myotubes [32], was confirmed in the present study.

It has previously been demonstrated that cultured myotubes retain their in vivo muscle phenotype in terms of insulin sensitivity [29,30] and mitochondrial lipid metabolism [31]. In the current study, we confirm that also after the procedure of serum shock, mitochondrial function was reduced in type 2 diabetic patients compared to obese controls (supplemental figure 2). Despite this, we did not find any differences in circadian rhythmicity in the core molecular clock components BMAL1, CRY1 and PER2 between donor groups, which could indicate that the core molecular clock is not affected in muscle of type 2 diabetic patients, 
as was shown in temporal series of human white adipose tissue biopsies [36]. However, circadian rhythmicity was affected by the donor characteristics for REVERBA/B and for SIRT1 and NAMPT. Thus, circadian rhythmicity for REVERBA/B was detected in all groups except for type 2 diabetes patients. Furthermore, interestingly, we could only confirm rhythmicity for NAMPT and SIRT1 expression in human primary myotubes derived from endurancetrained athletes. As SIRT1 and NAMPT have been shown to be associated to metabolic health [37-39] it is interesting that intrinsic rhythmicity is less robust in sedentary lean, obese and T2D donor cells. Furthermore, it should be noted that all cells were cultured under optimized and constant cell culture conditions. Given the differences in metabolic flexibility between diabetic patients and (un)trained healthy lean subjects, it may be that the differences in circadian rhythmicity as measured in the current model may be underestimated. In that context, it may be interesting to expose synchronized skeletal myotubes with excess palmitate or glucose substrates and investigate whether circadian rhythmicity is more pronouncedly affected under such diabetogenic conditions.

We did demonstrate rhythmicity of regulatory genes in glucose and lipid metabolism or mitochondrial biogenesis using JTK_CYCLE, but these rhythms were not robust. This contrasts to findings in mouse liver $[7,40,41]$ as well as heart and skeletal muscle [42]. Further, murine skeletal muscle was shown to maintain rhythms of glucose metabolites and corresponding rate-limiting enzymes, such as GLUT4, HKII and PDK4 [25]. However, we did not observe rhythmic expression of HKII, GYSI or GSK3B mRNA in synchronized human myotubes of all groups. In addition, no circadian expression of IRS1 was detected, in contrast to murine muscle and liver [43]. Whilst lipid metabolism is highly diurnal in mouse liver, white adipose tissue and aorta, only moderate rhythm was detected in the present study for DGAT1 $[10,44]$ and PPARa $[10,12]$ or PPARd [11]. In line with the absence of oscillations in metabolic gene expression, the mitochondrial gene ANT is expressed with minimal variation over time. It should be noted that even when rhythmicity in metabolic gene expression was observed, the amplitude of the rhythm was very low in comparison to the clock genes.

In conclusion, we demonstrate, that circadian components of the molecular clock, namely REVERBA/B, SIRT1 and NAMPT, that are associated with mitochondrial function and 
metabolic health, showed lack of circadian rhythmicity in human primary myotubes from type 2 diabetic patients. Modest circadian rhythmicity was observed in few downstream genes of the molecular clock system, such as metabolic genes involved in glucose and lipid metabolism. Future studies are needed to examine if circadian rhythmicity can also be observed in physiological measures such as insulin sensitivity or mitochondrial function.

\section{Methods:}

\section{Study design and subject characteristics}

In the present study, we included human primary myotubes from subjects that had previously participated in our studies $[45,46]$. We cultured human primary myotubes from the following four groups: 1) young endurance trained athletes (TL) and 2) their agematched young, lean sedentary controls (UL), and 3) healthy, obese subjects (OB) and 4) BMI- and age-matched type 2 diabetic patients (T2D) (table 1). Inclusion criteria for endurance trained athletes were performing endurance exercise (long-distance running, cycling, swimming) at least three times a week for the past 2 years and a VO2max $>55$ $\mathrm{ml} / \mathrm{min} / \mathrm{kg}$; all other subjects performed less than $1 \mathrm{~h}$ of exercise per week for the past 2 years. Type 2 diabetic patients were diagnosed at least 1 year prior to the study, were noninsulin dependent, were well-controlled $(\mathrm{HbA} 1 \mathrm{c}<64 \mathrm{mmol} / \mathrm{mol})$, and had no diabetesrelated comorbidities. All type 2 diabetes patients had been treated with metformin or metformin + sulfonylureas for at least 6 months. All studies were approved by the local Medical Ethical Committee of Maastricht University according to the declaration of Helsinki principles of 1975, as revised in 1983. All participants gave written informed consent.

\begin{tabular}{|c|c|c|c|c|c|c|c|c|c|c|c|c|c|}
\hline & & \multicolumn{3}{|c|}{ Trained lean } & \multicolumn{3}{|c|}{ Untrained Lean } & \multicolumn{3}{|c|}{ Obese } & \multicolumn{3}{|c|}{ Type 2 Diabetic } \\
\hline & & Average & \pm SEM & $\mathrm{N}$ & Average & \pm SEM & $\mathrm{N}$ & Average & \pm SEM & $\mathrm{N}$ & Average & \pm SEM & $\mathrm{N}$ \\
\hline Age & [yr] & 22.0 & 1.0 & 3 & 22.3 & 1.2 & 3 & 57.3 & 12.9 & 3 & 59.0 & 7.0 & 3 \\
\hline BMI & {$\left[\mathrm{kg} / \mathrm{m}^{2}\right]$} & 19.2 & 1.4 & 3 & 21.3 & 1.4 & 3 & 30.2 & 1.4 & 3 & 30.3 & 0.3 & 3 \\
\hline $\mathrm{M}$-Value $40 \mathrm{mU}$ & {$\left[\mathrm{mmol} / \mathrm{kg}_{\mathrm{FFM}} / \mathrm{min}\right]$} & 76.1 & 5.9 & 3 & 50.6 & 13.6 & 3 & 26.8 & 3.0 & 3 & 19.5 & 10.8 & 3 \\
\hline Fat mass & [\%] & 13.8 & 0.3 & 3 & 15.5 & 5.4 & 3 & 30.9 & 4.9 & 3 & 34.7 & 3.4 & 3 \\
\hline VO2-max & {$[\mathrm{mL} / \mathrm{min} / \mathrm{kg}]$} & 61.4 & 5.1 & 3 & 41.8 & 0.8 & 3 & 28.4 & 3.7 & 3 & 27.5 & 4.3 & 3 \\
\hline
\end{tabular}




\section{Hyperinsulinemic-euglycemic clamp}

Peripheral insulin sensitivity was measured by a two-step hyperinsulinemic-euglycemic clamp, according to Sparks et al [45]. The M-value was calculated as the glucose infusion rate required to maintain euglycemia $(5.5 \mathrm{mmol} / \mathrm{l})$ under hyperinsulinemic conditions (insulin infusion at $40 \mathrm{mU} / \mathrm{m}^{2} / \mathrm{min}$ ) and was corrected for fat-free mass (FFM).

\section{Maximal aerobic exercise}

A stepwise progressive exercise test until exhaustion was performed on a stationary bike to measure whole body oxygen uptake as a reflection of physical fitness. Simultaneous breath gas analysis to measure maximal oxygen $\left(\mathrm{VO}_{2} \max \right)$ uptake and carbon oxide production $\left(\mathrm{VCO}_{2}\right)$ was performed under standardized laboratory conditions. Exercise was stopped when the subjects were unable to maintain cadence $>60$ RPM, heart rate exceeded 220 minus age or at a respiratory exchange ratio $>1.10$.

\section{Primary muscle cell cultures}

As described before [45], muscle biopsy specimens were taken from the musculus vastus lateralis according to Bergström [47] and processed the same day for cell culture. All biopsies were obtained in the overnight fasted state between 8 to 9 AM. Primary skeletal muscle cell cultures were established by isolating and growing needle biopsy derived satellite cells in media supplemented with 16\% FBS (Gibco, Thermo Fisher Scientific, Waltham, USA) at $37^{\circ} \mathrm{C}$ and $5 \% \mathrm{CO}_{2}$. Differentiation to multi-nucleated skeletal myotubes was induced by seeding mono-nucleated satellite cells confluently in differentiation media (passage <5); Minimum Essential Medium a (Gibco, Thermo Fisher Scientific, Waltham, USA) supplemented with 2\% FBS (Gibco, Thermo Fisher Scientific, Waltham, USA) and fetuin (Sigma-Aldrich, St. Louis, USA). Every other day differentiation media was changed.

\section{In-vitro synchronization of primary myotubes}

On day seven of differentiation, multi-nucleated skeletal myotubes were synchronized by serum shock [35]. Serum shock consisted of a two-hour incubation with fresh differentiation media, supplemented with 50\% horse serum (Gibco, Thermo Fisher Scientific, Waltham, USA). After serum shock, cultures were washed and supplemented with fresh differentiation media. All individual cultures of the four donor groups were synchronized on the same day. 
Cultures were harvested $0,16,20,24,28,32,36,40,44,48,52,56,64$, or 72 hours after synchronization by placing the culture plate on ice and washing thoroughly with Dulbecco's Phosphate-Buffered Saline (Gibco, Thermo Fisher Scientific, Waltham, USA). Supernatant was immediately removed and cell lysis was initiated.

\section{Transcript quantification and normalization}

RNA isolation was performed on-dish by TRIzol lysis (Qiagen, Hilden, Germany). RNA was further purified by the RNeasy kit from Qiagen (Hilden, Germany). The quality and yield of RNA was assessed using a NanoDrop spectrophotometer (Thermo Fisher Scientific, Waltham, USA). The high-capacity RNA-to-cDNA kit from Applied Biosystems (Foster City, USA) was used for transcribing $0.5 \mu \mathrm{g}$ RNA to cDNA. Transcript abundance was determined using a 7900HT Fast Real-Time PCR System (Applied Biosystems, Waltham, USA). Three housekeeping genes (HKG), namely RPL26, GUSB and CYPB, were identified to be stably expressed in synchronized multi-nucleated myotubes throughout time (supplemental figure 1) by both GeneNorm [48] and Bestkeeper [49]. To further minimalize the variability in housekeeping gene normalization, the geometric mean of the three housekeeping genes, which showed strong robustness in time, was used. Therefore, the geometric mean was used as the internal reference for the comparative gene expression analysis in the remainder of the study. Thus, each sample was normalized to the corresponding sample at time of serum shock by comparative gene expression analysis.

\section{Mitochondrial function - Oxygen Consumption Rate (OCR) measurements}

To determine whether cultures of the four donor groups kept the donor phenotype, human primary myotube were plated and differentiated on a Seahorse Bioscience 96-well plate. One hour prior to measurement, differentiation medium was changed to Seahorse Biosciences XF media according to the XF Cell Mito Stress Test Kit (Seahorse Bioscience, USA, North Billerica). Oligomycin, FCCP and rotenone with antimycin A was added to the cells to determine the oxygen consumption rates for basal mitochondrial respiration, maximal respiration and mitochondrial proton leak (supplemental figure 2). OCR was corrected for non-mitochondrial respiration (rotenone with antimycin A respiration). 


\section{Statistical analysis}

Results are presented as means \pm SEM derived from three independent cultures of each donor group. Gene expression was tested for rhythmicity using the JTK_CYCLE package for R in Windows 8 [50]; for this, data from 4-hourly sampled time points (between $16 \mathrm{~h}$ and $56 \mathrm{~h}$ ) determining the best fit for 20,24 or 28 hours of period length was used. In case JTK_CYCLE indicated rhythmicity, we tested whether gene expression between time points $16 \mathrm{~h}$ to $56 \mathrm{~h}$ was statistically significant by one-way ANOVA, followed by Bonferroni post-hoc analysis for determination of peak and nadir in rhythmic expression profiles. If significant rhythmicity was observed, two-way ANOVA was also performed to detect group differences in circadian rhythmicity between groups. A p-value $<0.05$ was considered statistically significant. Statistical analyses were performed using the statistical program SPSS 23.0 for Mac OS X. 


\section{Supplemental figures:}
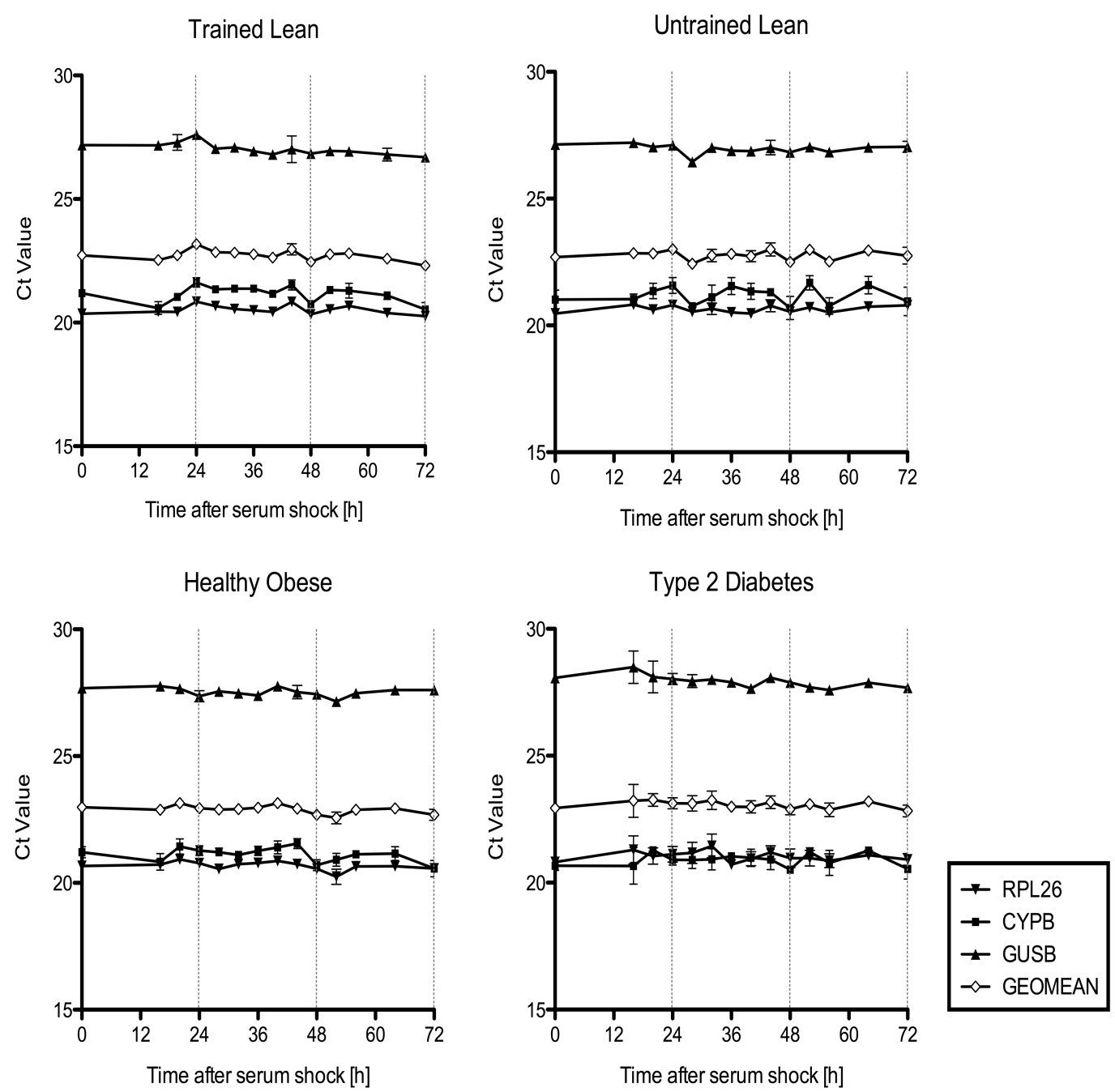

Supplemental Figure 1: Temporal gene expression stability of housekeeping genes. Gene Expression of the housekeeping genes RPL26 (reverse black triangles), GUSB (black triangle) and CYPB (black squares) in time after serum shock (hours). The geometric mean (open diamonds) consists of the raw ct-values of all three housekeeping genes per time point. Data are mean \pm SEM. $n=3$ for each group. 
Basal Respiration

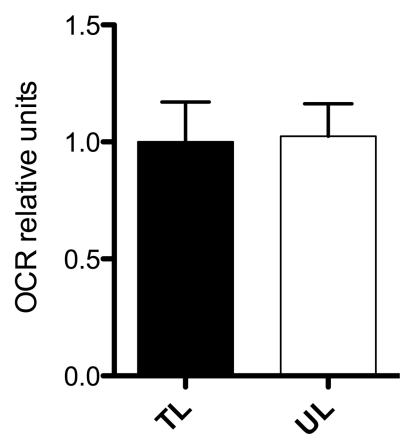

Basal Respiration

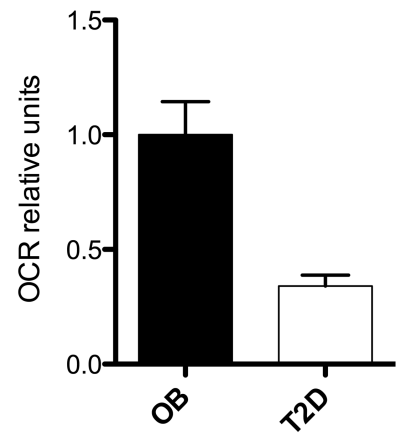

Maximal Respiration

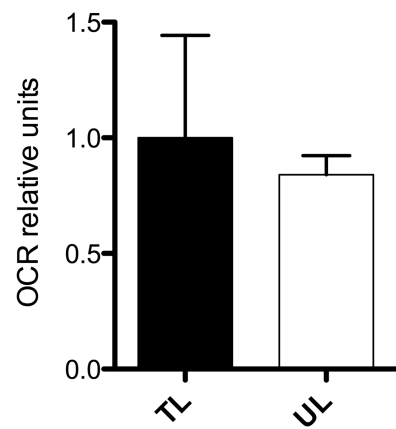

Maximal Respiration

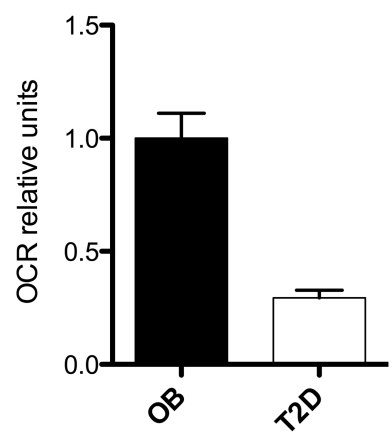

Mitochondrial Proton Leak

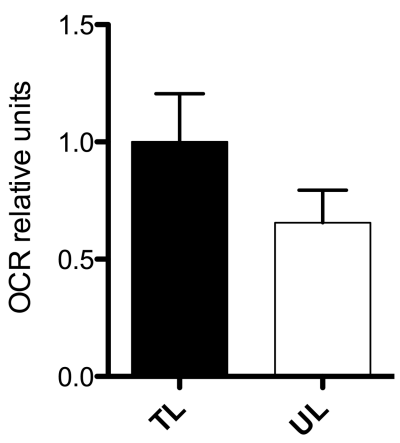

Mitochondrial Proton Leak

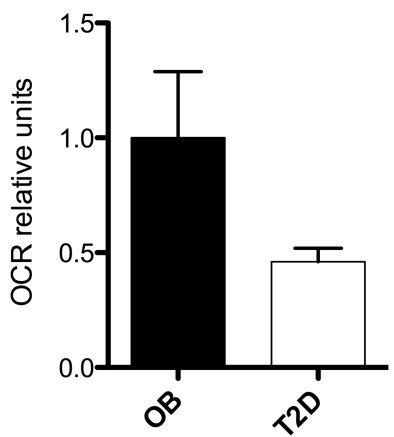

UL - untrained lean

TL - trained lean $O B$ - healthy obese T2D - type 2 diabetes

Supplemental Figure 2: Respiratory capacity of donor phenotypes in cultured myotubes.

The oxygen consumption rate (OCR) of the mitochondrial basal respiration $(A)$, maximal respiration (B) and mitochondrial proton leak $(C)$ are depicted relative to the control group ( $T L$ vs UL; $O B$ vs T2D). OCR is corrected for non-mitochondrial respiration (antimycin $A$ and rotenone). Data are mean \pm SEM. 

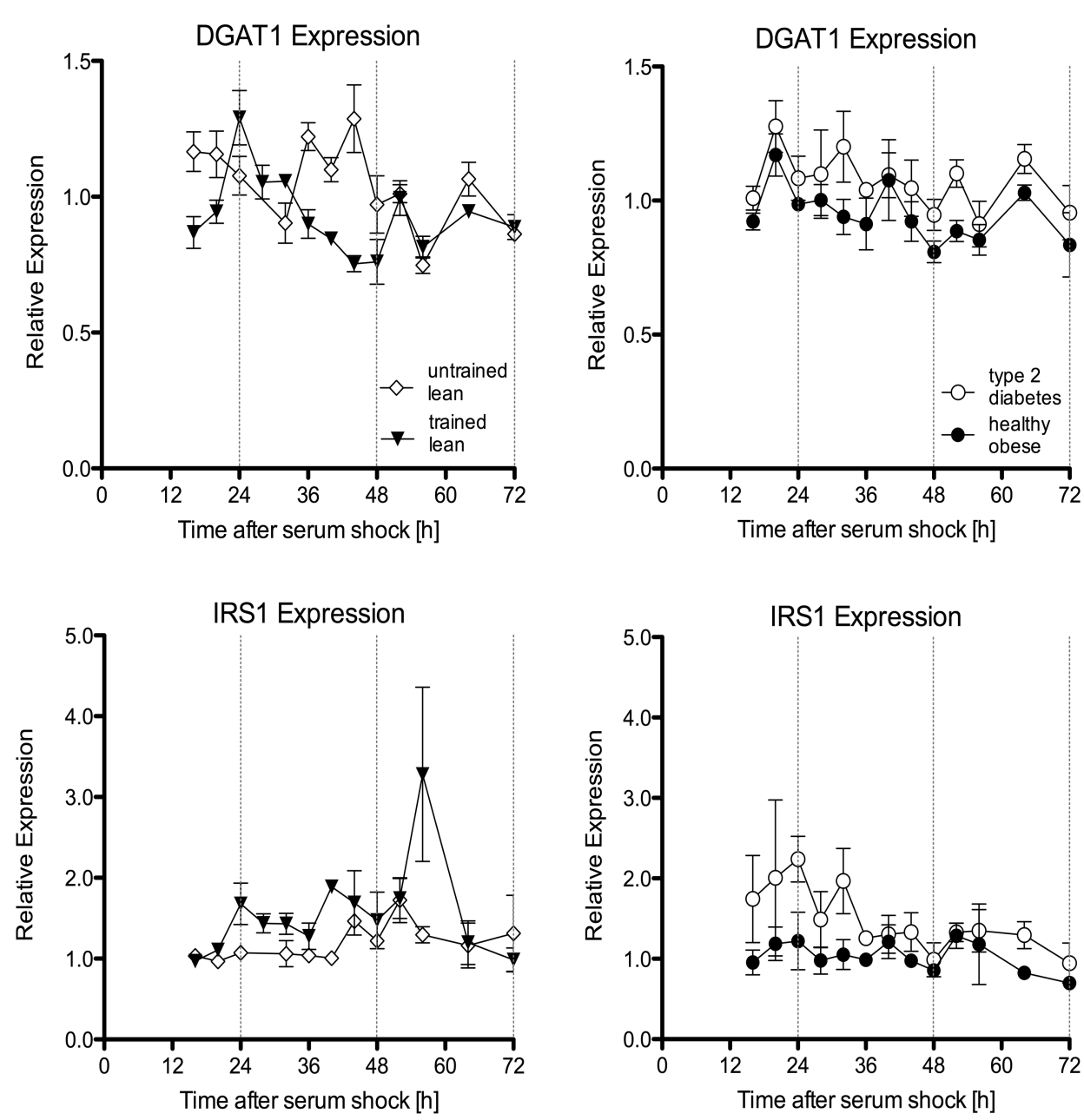

\section{Supplemental Figure 3: Expression profiles of IRS1 and DGAT1.}

The relative transcript abundance of IRS1 that encodes a protein involved in intracellular insulin signaling and DGAT1 that encodes a protein involved in triglyceride synthesis was quantified by RT-PCR and normalized to the corresponding geometric mean of RPL26, CYPB and GUSB. Each value consists of the average of the independent cultures from three different donors. The value of the sample taken immediately after serum shock (Oh) was normalized to 1. Expression profiles of synchronized differentiated primary myotube cultures are plotted as follows: trained lean (black triangle) against untrained lean (open diamond) and obese (black circle) against type 2 diabetics (open circle). Data are mean \pm SEM 


\section{References}

[1] Defronzo, R.A., D. Simonson, E. Ferrannini, and E. Barrett, 1981. Insulin resistance: a universal finding in diabetic states. Bull Schweiz Akad Med Wiss:223-238.

[2] Kelley, D.E., J. He, E.V. Menshikova, and V.B. Ritov, 2002. Dysfunction of mitochondria in human skeletal muscle in type 2 diabetes. Diabetes 51(10):29442950.

[3] Phielix, E., V.B. Schrauwen-Hinderling, M. Mensink, E. Lenaers, R. Meex, J. Hoeks, et al., 2008. Lower intrinsic ADP-stimulated mitochondrial respiration underlies in vivo mitochondrial dysfunction in muscle of male type 2 diabetic patients. Diabetes 57(11):2943-2949.

[4] Kelley, D.E. and L.J. Mandarino, 2000. Fuel selection in human skeletal muscle in insulin resistance: a reexamination. Diabetes 49(5):677-683.

[5] Lowrey, P.L. and J.S. Takahashi, 2004. Mammalian circadian biology: elucidating genome-wide levels of temporal organization. Annu Rev Genomics Hum Genet $5(407-441$.

[6] Cho, H., X. Zhao, M. Hatori, R.T. Yu, G.D. Barish, M.T. Lam, et al., 2012. Regulation of circadian behaviour and metabolism by REV-ERB-alpha and REV-ERB-beta. Nature 485(7396):123-127.

[7] Nakahata, Y., S. Sahar, G. Astarita, M. Kaluzova, and P. Sassone-Corsi, 2009. Circadian control of the NAD+ salvage pathway by CLOCK-SIRT1. Science 324(5927):654-657.

[8] Gallego, M. and D.M. Virshup, 2007. Post-translational modifications regulate the ticking of the circadian clock. Nat Rev Mol Cell Biol 8(2):139-148.

[9] Yoo, S.H., J.A. Mohawk, S.M. Siepka, Y. Shan, S.K. Huh, H.K. Hong, et al., 2013. Competing E3 ubiquitin ligases govern circadian periodicity by degradation of CRY in nucleus and cytoplasm. Cell 152(5):1091-1105.

[10] Adamovich, Y., L. Rousso-Noori, Z. Zwighaft, A. Neufeld-Cohen, M. Golik, J. KrautCohen, et al., 2014. Circadian clocks and feeding time regulate the oscillations and levels of hepatic triglycerides. Cell Metab 19(2):319-330.

[11] Liu, S., J.D. Brown, K.J. Stanya, E. Homan, M. Leidl, K. Inouye, et al., 2013. A diurnal serum lipid integrates hepatic lipogenesis and peripheral fatty acid use. Nature 502(7472):550-554.

[12] Rudic, R.D., P. McNamara, D. Reilly, T. Grosser, A.M. Curtis, T.S. Price, et al., 2005. Bioinformatic analysis of circadian gene oscillation in mouse aorta. Circulation 112(17):2716-2724.

[13] Yang, X., M. Downes, R.T. Yu, A.L. Bookout, W. He, M. Straume, et al., 2006. Nuclear receptor expression links the circadian clock to metabolism. Cell 126(4):801-810.

[14] Andrews, J.L., X. Zhang, J.J. McCarthy, E.L. McDearmon, T.A. Hornberger, B. Russell, et al., 2010. CLOCK and BMAL1 regulate MyoD and are necessary for maintenance of skeletal muscle phenotype and function. Proc Natl Acad Sci U S A 107(44):1909019095.

[15] Hara, R., K. Wan, H. Wakamatsu, R. Aida, T. Moriya, M. Akiyama, et al., 2001. Restricted feeding entrains liver clock without participation of the suprachiasmatic nucleus. Genes Cells 6(3):269-278.

[16] Feillet, C.A., U. Albrecht, and E. Challet, 2006. "Feeding time" for the brain: a matter of clocks. J Physiol Paris 100(5-6):252-260. 
[17] lijima, M., S. Yamaguchi, G.T. van der Horst, X. Bonnefont, H. Okamura, and S. Shibata, 2005. Altered food-anticipatory activity rhythm in Cryptochrome-deficient mice. Neurosci Res 52(2):166-173.

[18] Wolff, G. and K.A. Esser, 2012. Scheduled exercise phase shifts the circadian clock in skeletal muscle. Med Sci Sports Exerc 44(9):1663-1670.

[19] Marcheva, B., K.M. Ramsey, E.D. Buhr, Y. Kobayashi, H. Su, C.H. Ko, et al., 2010. Disruption of the clock components CLOCK and BMAL1 leads to hypoinsulinaemia and diabetes. Nature 466(7306):627-631.

[20] Lamia, K.A., K.F. Storch, and C.J. Weitz, 2008. Physiological significance of a peripheral tissue circadian clock. Proc Natl Acad Sci U S A 105(39):15172-15177.

[21] Lee, J., M.S. Kim, R. Li, V.Y. Liu, L. Fu, D.D. Moore, et al., 2011. Loss of Bmal1 leads to uncoupling and impaired glucose-stimulated insulin secretion in beta-cells. Islets 3(6).

[22] Rudic, R.D., P. McNamara, A.M. Curtis, R.C. Boston, S. Panda, J.B. Hogenesch, et al., 2004. BMAL1 and CLOCK, two essential components of the circadian clock, are involved in glucose homeostasis. PLoS Biol 2(11):e377.

[23] Delezie, J., S. Dumont, H. Dardente, H. Oudart, A. Grechez-Cassiau, P. Klosen, et al., 2012. The nuclear receptor REV-ERBalpha is required for the daily balance of carbohydrate and lipid metabolism. FASEB J 26(8):3321-3335.

[24] Lamia, K.A., S.J. Papp, R.T. Yu, G.D. Barish, N.H. Uhlenhaut, J.W. Jonker, et al., 2011. Cryptochromes mediate rhythmic repression of the glucocorticoid receptor. Nature 480(7378):552-556.

[25] Dyar, K.A., S. Ciciliot, L.E. Wright, R.S. Bienso, G.M. Tagliazucchi, V.R. Patel, et al., 2014. Muscle insulin sensitivity and glucose metabolism are controlled by the intrinsic muscle clock. Mol Metab 3(1):29-41.

[26] Gomez-Abellan, P., J.J. Hernandez-Morante, J.A. Lujan, J.A. Madrid, and M. Garaulet, 2008. Clock genes are implicated in the human metabolic syndrome. Int J Obes (Lond) 32(1):121-128.

[27] Zanquetta, M.M., M.L. Correa-Giannella, D. Giannella-Neto, P.A. Alonso, L.M. Guimaraes, A. Meyer, et al., 2012. Expression of clock genes in human subcutaneous and visceral adipose tissues. Chronobiol Int 29(3):252-260.

[28] Pivovarova, O., K. Jurchott, N. Rudovich, S. Hornemann, L. Ye, S. Mockel, et al., 2015. Changes of Dietary Fat and Carbohydrate Content Alter Central and Peripheral Clock in Humans. J Clin Endocrinol Metab 100(6):2291-2302.

[29] Green, C.J., M. Pedersen, B.K. Pedersen, and C. Scheele, 2011. Elevated NF-kappaB activation is conserved in human myocytes cultured from obese type 2 diabetic patients and attenuated by AMP-activated protein kinase. Diabetes 60(11):28102819.

[30] Bourlier, V., C. Saint-Laurent, K. Louche, P.M. Badin, C. Thalamas, I. de Glisezinski, et al., 2013. Enhanced glucose metabolism is preserved in cultured primary myotubes from obese donors in response to exercise training. J Clin Endocrinol Metab 98(9):3739-3747.

[31] Gaster, M., A.C. Rustan, V. Aas, and H. Beck-Nielsen, 2004. Reduced lipid oxidation in skeletal muscle from type 2 diabetic subjects may be of genetic origin: evidence from cultured myotubes. Diabetes 53(3):542-548. 
[32] Perrin, L., U. Loizides-Mangold, S. Skarupelova, P. Pulimeno, S. Chanon, M. Robert, et al., 2015. Human skeletal myotubes display a cell-autonomous circadian clock implicated in basal myokine secretion. Mol Metab 4(11):834-845.

[33] Bonaconsa, M., G. Malpeli, A. Montaruli, F. Carandente, G. Grassi-Zucconi, and M. Bentivoglio, 2014. Differential modulation of clock gene expression in the suprachiasmatic nucleus, liver and heart of aged mice. Exp Gerontol 55(70-79.

[34] Bjarnason, G.A., R.C. Jordan, P.A. Wood, Q. Li, D.W. Lincoln, R.B. Sothern, et al., 2001. Circadian expression of clock genes in human oral mucosa and skin: association with specific cell-cycle phases. Am J Pathol 158(5):1793-1801.

[35] Balsalobre, A., F. Damiola, and U. Schibler, 1998. A serum shock induces circadian gene expression in mammalian tissue culture cells. Cell 93(6):929-937.

[36] Otway, D.T., S. Mantele, S. Bretschneider, J. Wright, P. Trayhurn, D.J. Skene, et al., 2011. Rhythmic diurnal gene expression in human adipose tissue from individuals who are lean, overweight, and type 2 diabetic. Diabetes 60(5):1577-1581.

[37] Pedersen, S.B., J. Olholm, S.K. Paulsen, M.F. Bennetzen, and B. Richelsen, 2008. Low Sirt1 expression, which is upregulated by fasting, in human adipose tissue from obese women. Int J Obes (Lond) 32(8):1250-1255.

[38] Rutanen, J., N. Yaluri, S. Modi, J. Pihlajamaki, M. Vanttinen, P. Itkonen, et al., 2010. SIRT1 mRNA expression may be associated with energy expenditure and insulin sensitivity. Diabetes 59(4):829-835.

[39] Costford, S.R., S. Bajpeyi, M. Pasarica, D.C. Albarado, S.C. Thomas, H. Xie, et al., 2010. Skeletal muscle NAMPT is induced by exercise in humans. Am J Physiol Endocrinol Metab 298(1):E117-126.

[40] Eckel-Mahan, K.L., V.R. Patel, S. de Mateo, R. Orozco-Solis, N.J. Ceglia, S. Sahar, et al., 2013. Reprogramming of the circadian clock by nutritional challenge. Cell 155(7):1464-1478.

[41] Peek, C.B., A.H. Affinati, K.M. Ramsey, H.Y. Kuo, W. Yu, L.A. Sena, et al., 2013. Circadian clock NAD+ cycle drives mitochondrial oxidative metabolism in mice. Science 342(6158):1243417.

[42] Um, J.H., J.S. Pendergast, D.A. Springer, M. Foretz, B. Viollet, A. Brown, et al., 2011. AMPK regulates circadian rhythms in a tissue- and isoform-specific manner. PLoS One 6(3):e18450.

[43] Harfmann, B.D., E.A. Schroder, and K.A. Esser, 2015. Circadian rhythms, the molecular clock, and skeletal muscle. J Biol Rhythms 30(2):84-94.

[44] Shostak, A., J. Meyer-Kovac, and H. Oster, 2013. Circadian regulation of lipid mobilization in white adipose tissues. Diabetes 62(7):2195-2203.

[45] Sparks, L.M., M. Bosma, B. Brouwers, T. van de Weijer, L. Bilet, G. Schaart, et al., 2014. Reduced incorporation of fatty acids into triacylglycerol in myotubes from obese individuals with type 2 diabetes. Diabetes 63(5):1583-1593.

[46] Vosselman, M.J., J. Hoeks, B. Brans, H. Pallubinsky, E.B. Nascimento, A.A. van der Lans, et al., 2015. Low brown adipose tissue activity in endurance-trained compared with lean sedentary men. Int J Obes (Lond) 39(12):1696-1702.

[47] Bergstrom, J., 1975. Percutaneous needle biopsy of skeletal muscle in physiological and clinical research. Scand J Clin Lab Invest 35(7):609-616.

[48] Vandesompele, J., K. De Preter, F. Pattyn, B. Poppe, N. Van Roy, A. De Paepe, et al., 2002. Accurate normalization of real-time quantitative RT-PCR data by geometric averaging of multiple internal control genes. Genome Biol 3(7):RESEARCH0034. 
[49] Pfaffl, M.W., A. Tichopad, C. Prgomet, and T.P. Neuvians, 2004. Determination of stable housekeeping genes, differentially regulated target genes and sample integrity: BestKeeper--Excel-based tool using pair-wise correlations. Biotechnol Lett 26(6):509-515.

[50] Miyazaki, M., E. Schroder, S.E. Edelmann, M.E. Hughes, K. Kornacker, C.W. Balke, et al., 2011. Age-associated disruption of molecular clock expression in skeletal muscle of the spontaneously hypertensive rat. PLoS One 6(11):e27168. 


\section{CHAPTER 4}

Characterization of temporal mitochondrial respiration and protein content in synchronized human primary myotubes of lean, obese and T2D donors

\section{Authors}

Jan Hansen, Silvie Timmers, Matthijs KC Hesselink, Patrick Schrauwen 


\begin{abstract}
Day-night rhythms in human energy metabolism and mitochondrial respiration have been demonstrated (CHAPTER 5). So far it is unknown whether such rhythms are induced by behavioral and environmental cycles or governed by a circadian molecular clock. To investigate this, we used human primary myotubes to examine whether rhythms in muscle mitochondrial respiration indeed originate from intrinsic circadian rhythms. Additionally, we explored whether mitochondrial respiration over time is altered in cultures of donors with obesity or type 2 diabetes compared to those of metabolically healthy donors. Therefore, human primary myotubes were obtained from skeletal muscle biopsies of four groups: type 2 diabetes patients and their BMI- and age-matched obese controls and from lean, healthy and young endurance trained athletes and their age-matched sedentary controls.

In synchronized human primary myotubes mitochondrial respiration was measured 6-hourly using high resolution respirometry over a time-course of 30 hours. In none of the groups mitochondrial respiration resembled rhythmicity. However, we observed a markedly reduced mitochondrial respiration in cultures of T2D patients. Interestingly, in cultures of all groups we encountered a pronounced rhythm in total protein content.

In conclusion, our data demonstrates that human skeletal muscle cells in culture do not display an intrinsic circadian rhythmicity in mitochondrial respiration. The reason for the $24 \mathrm{~h}$ variation in total cellular protein content could not be revealed from the present study and needs future investigation.
\end{abstract}




\section{Introduction:}

The human sleep-wake cycle is associated with fluctuations in substrate metabolism as food consumption and physical activity occur predominantly during the wake phase. In order to resonate with these daily nutritional challenges, peripheral organs such as liver, white adipose tissue and skeletal muscle play important roles in maintaining whole body energy balance. In a recent human study, we demonstrated that in young healthy male volunteers, predominantly carbohydrates are utilized during the wake phase, while substrate utilization switches progressively to fat oxidation during the sleep phase [1]. Surprisingly, we found that not only whole body energy expenditure was highest in the late evening hours, but also skeletal muscle mitochondrial oxidative capacity revealed highest values in the late evening hours (figure 1). In fact, mitochondrial oxidative capacity displayed 24-hour oscillations, although it remains elusive whether this rhythm is imposed by circadian- or behavioral cycles, such as food intake and physical activity (figure 1).

To date, in animal studies, several circadian genes from the molecular clock have been causally linked to the regulation of mitochondrial metabolism [2], mitochondrial- integrity, density [3] and dynamics [4]. In addition, we recently found in a study using human primary myocytes [5], that the rhythmicity of two key-regulators of mitochondrial biogenesis and the molecular clock, REVERBA and SIRT1, was affected by the characteristics of the donors, being either overweight or type 2 diabetic, when compared to healthy controls. Noteworthy, in vivo and ex vivo mitochondrial oxidative capacity is diminished in both subject groups when compared to healthy lean controls.

So far, it is unknown if the rhythmicity in mitochondrial function that we observed in human skeletal muscle is driven by circadian or behavioral (food, activity) rhythmicity. Therefore, in the current study we aimed to investigate mitochondrial rhythmicity in skeletal muscle isolated from behavioral cues, as outlined in figure 1. Hence, we synchronized cultured human primary skeletal myotubes of young lean donors, obese subjects and type 2 diabetes patients, and examined if the previously observed rhythmicity in in vivo mitochondrial function is maintained in skeletal muscle cells isolated from human volunteers. 


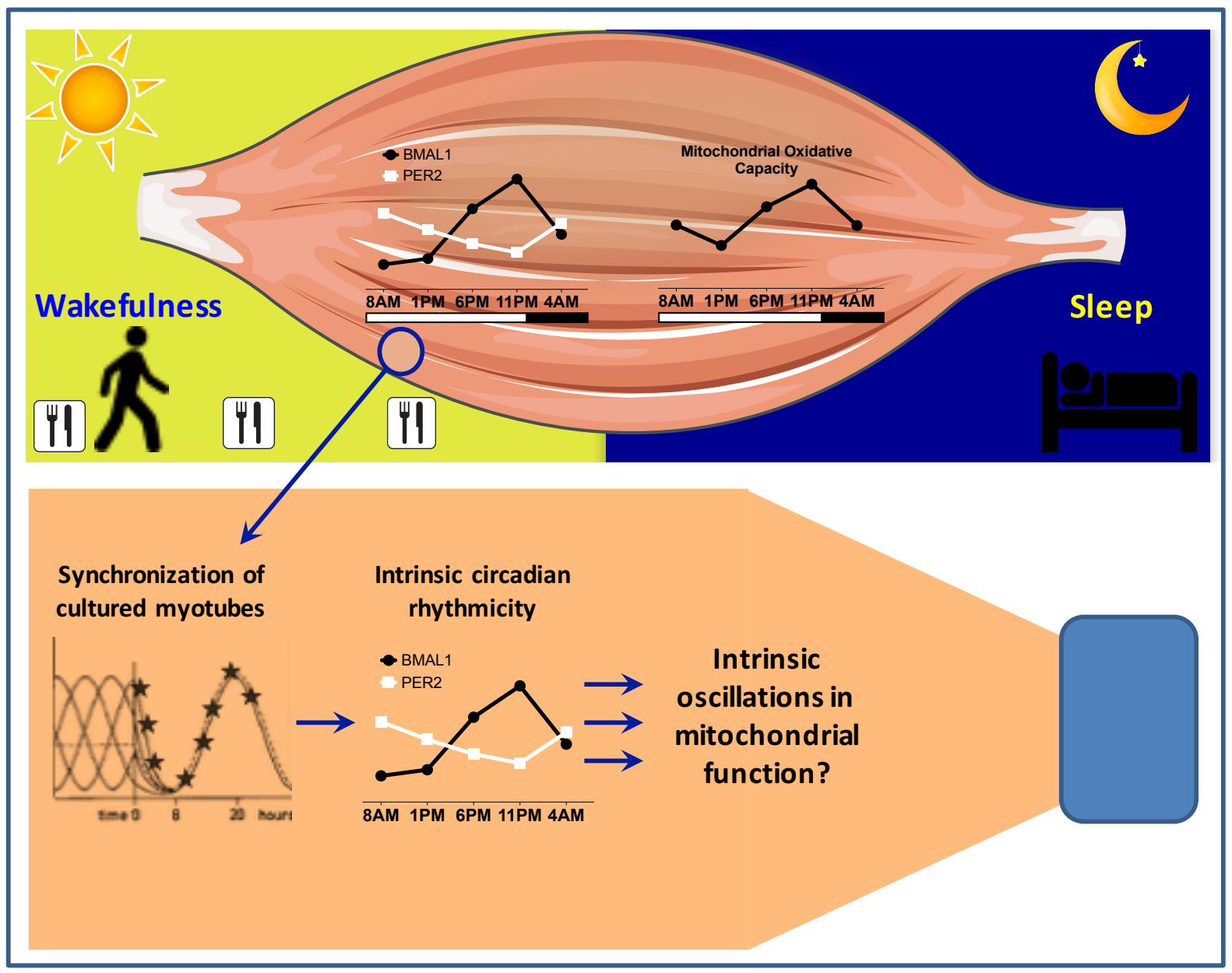

Figure 1: Is mitochondrial function under circadian control?

Upper panel displays day-night rhythmicity of human skeletal muscle in mRNA and mitochondrial function [1]. Light, Sleep-wake cycles, fast-fed cycles as well as physical activity are strong time cues for maintaining day-night rhythms in all mammals. In human skeletal muscle tissue, molecular clock genes oscillate in a 24-hour rhythm to orchestrate the timing of cellular functions. Whether the observed rhythms in mitochondrial oxidative capacity are intrinsically circadian or induced by the behavioral cycles remains elusive. Lower Panel demonstrates effect of synchronization by serum shock in cultured cells (adapted from [6]). Once synchronized, the molecular clock continues to oscillate for several days in human primary muscle cultures. Isolated from all physiological cycles and signals, rhythms in cellular funtion - such as mitochondrial oxidative capacity - would be considered to be intrinsic circadian rhythms. 


\section{Methods:}

\section{Study design and subject characteristics}

We included primary myotubes from subjects that had previously participated in our studies $[7,8]$. Human primary myotubes were cultured from the following four groups: 1) young endurance trained athletes (TL), 2) age-matched young, lean sedentary controls (UL), and 3) middle-aged healthy, obese subjects (OB) and 4) BMI- and age-matched type 2 diabetes patients (T2D) (Table 1). Endurance trained athletes were included when they were performing endurance exercise activities (long-distance running, cycling, swimming) at least three times a week for the past 2 years and had a VO2max > $55 \mathrm{ml} / \mathrm{min} / \mathrm{kg}$; all other subjects were performing less than 1 h of exercise per week for the past 2 years. Type 2 diabetes patients were diagnosed at least 1 year prior to the study, were non-insulin dependent, were well-controlled ( $\mathrm{HbA} 1 \mathrm{c}<64 \mathrm{mmol} / \mathrm{mol})$, and had no diabetes-related comorbidities. All type 2 diabetes patients had been treated with metformin or metformin + sulfonylureas for at least 6 months.

The metabolic phenotypes were determined as previously described [5]. In short, a hyperinsulinemic-euglygemic clamp was used to measure peripheral insulin sensitivity and a stepwise progressive exercise protocol was used to measure maximal aerobic capacity (VO2-max). All studies were approved by the local Medical Ethical Committee of Maastricht University according to the declaration of Helsinki principles. All participants gave their written informed consent.

\begin{tabular}{|c|c|c|c|c|c|c|c|c|c|c|c|c|c|}
\hline & & \multicolumn{3}{|c|}{ Trained lean } & \multicolumn{3}{|c|}{ Untrained Lean } & \multicolumn{3}{|l|}{ Obese } & \multicolumn{3}{|c|}{ Type 2 Diabetic } \\
\hline & & Average & \pm SEM & $\mathrm{N}$ & Average & \pm SEM & $\mathrm{N}$ & Average & \pm SEM & $\mathrm{N}$ & Average & \pm SEM & $\mathrm{N}$ \\
\hline Age & [yr] & 22.0 & 1.0 & 3 & 22.3 & 1.2 & 3 & 57.3 & 12.9 & 3 & 59.0 & 7.0 & 3 \\
\hline BMI & {$\left[\mathrm{kg} / \mathrm{m}^{2}\right]$} & 19.2 & 1.4 & 3 & 21.3 & 1.4 & 3 & 30.2 & 1.4 & 3 & 30.3 & 0.3 & 3 \\
\hline $\mathrm{M}$-Value $40 \mathrm{mU}$ & {$\left[\mathrm{mmol} / \mathrm{kg}_{\mathrm{FFM}} / \mathrm{min}\right]$} & 76.1 & 5.9 & 3 & 50.6 & 13.6 & 3 & 26.8 & 3.0 & 3 & 19.5 & 10.8 & 3 \\
\hline Fat mass & [\%] & 13.8 & 0.3 & 3 & 15.5 & 5.4 & 3 & 30.9 & 4.9 & 3 & 34.7 & 3.4 & 3 \\
\hline VO2-max & {$[\mathrm{mL} / \mathrm{min} / \mathrm{kg}]$} & 61.4 & 5.1 & 3 & 41.8 & 0.8 & 3 & 28.4 & 3.7 & 3 & 27.5 & 4.3 & 3 \\
\hline
\end{tabular}

\section{Primary muscle cell cultures}

As reported before [5], primary skeletal muscle cell cultures were established by isolating and culturing satellite cells derived from muscle biopsies. Differentiation to multi-nucleated skeletal myotubes was induced by seeding mono-nucleated satellite cells confluently in 
differentiation media (passage $<5$ ), consisting of minimum essential medium $\alpha$ (Gibco, Thermo Fisher Scientific, Waltham, USA) supplemented with $2 \%$ fetal bovine serum (Gibco, Thermo Fisher Scientific, Waltham, USA) and fetuin (Sigma-Aldrich, St. Louis, USA).

\section{Mitochondrial function - Oxygen Consumption Rate (OCR) measurements}

Human primary myotubes were differentiated for 7 days on Seahorse Bioscience 96-well plates before serum shock synchronization, as previously described [5]. Cultures were measured 18, 24, 30, 36, 42 and 48 hours after synchronization using the XF Cell Mito Stress Test Kit (Seahorse Bioscience, USA, North Billerica) to determine cellular respiration. After measuring baseline oxygen consumption rate (OCR), oligomycin - a complex $V$ inhibitor - is added to measure non-ATP-linked (leak) respiration. Addition of carbonyl cyanide-ptrifluoromethoxyphenylhydrazon (FCCP) - a mitochondrial membrane protonophore - was used to assess maximal capacity of the electron transport chain and maximal oxygen consumption rate. By using complex I and III inhibitors (rotenone and antimycin A, respectively) the electron transport chain was blocked, revealing non-mitochondrial derived respiration. Mitochondrial basal respiration is defined by subtracting non-mitochondrial respiration from baseline OCR. Coupled respiration is defined by subtracting oligomycin OCR from baseline OCR. Maximal mitochondrial respiration is determined by subtracting nonmitochondrial respiration from FCCP OCR. Mitochondrial spare capacity is determined by subtracting baseline OCR from FCCP OCR. Mitochondrial proton leak is determined by subtracting non-mitochondrial respiration from oligomycin OCR.

\section{Cellular protein content}

Immediately after the Seahorse measurement cells were washed twice with PBS before lysis with $0.1 \mathrm{M} \mathrm{NaOH}$ solution. In these cell lysates, the photometric Biorad DC Assay was used to determine absorption at $750 \mathrm{~nm}$ after 20 min incubation using the microplate reader SpectraMax M2 (Molecular Devices, LLC, California, USA). As reference for protein content of all total lysates, a protein standard curve of albumin solved in $0.1 \mathrm{M} \mathrm{NaOH}$ (SigmaAldrich, St. Louis, USA) was measured with the given concentrations: 2.5, 1.25, 0.625, $0.3125,0.15625$ and $0 \mathrm{mg} / \mathrm{ml}$. 


\section{Statistical analysis}

Results are presented as means \pm SEM derived from three independent cultures of each donor group. Cellular respiration was tested for statistical significant differences between the time points $18 \mathrm{~h}$ to $48 \mathrm{~h}$ and/or groups using two-way ANOVA. Statistical analyses were performed using the statistical program SPSS 23.0 for Mac OS X. 24-hour protein content was tested for rhythmicity performing COSINOR analysis, by non-linear fit to sine wave function in Graphpad Prism 5.0a on Mac OS X.

\section{Results}

\section{Oxygen consumption over time is consistently lower in T2D}

The aim of this study was to investigate whether intrinsic skeletal muscle mitochondrial metabolism displays circadian rhythmicity in synchronized human primary myotubes and whether such circadian rhythmicity would be affected by the donor group. Two-way ANOVA revealed no time nor time*group interaction effect in uncorrected oxygen consumption rates (figure 2) (TL p>0.05, UL $p>0.05, O B p>0.05, T 2 D p>0.05$ ). However, a group effect was observed for OCR, showing that oxygen consumption rates were $~ 50 \%$ lower in cells of T2D donors (figure 2) when compared to OCR in cells of $T L, U L$ and $O B$, for all respiration states (B_OCR $p<0.001$, Oligo_OCR $p<0.001$, FCCP_OCR $p<0.001$, R.AA_OCR $p<0.001$ ).

\section{Mitochondrial respiratory capacity is not rhythmic}

We next adjusted OCR for non-mitochondrial respiration to determine parameters of mitochondrial respiration (figure 3). As for uncorrected OCRs, baseline mitochondrial respiration rate, maximal respiration, mitochondrial spare capacity, proton leak respiration and coupled respiration did not show a significant time effect in any of the donor groups (two-way ANOVA, TL p>0.05, UL p>0.05, OB p>0.05, T2D p>0.05). However, two-way ANOVA revealed a significant group effect, with lowest respiration values in type 2 diabetes patients (B_OCR $p<0.001$, Oligo_OCR $p<0.001$, FCCP_OCR $p<0.001$, R.AA_OCR $p<0.001$ ). 

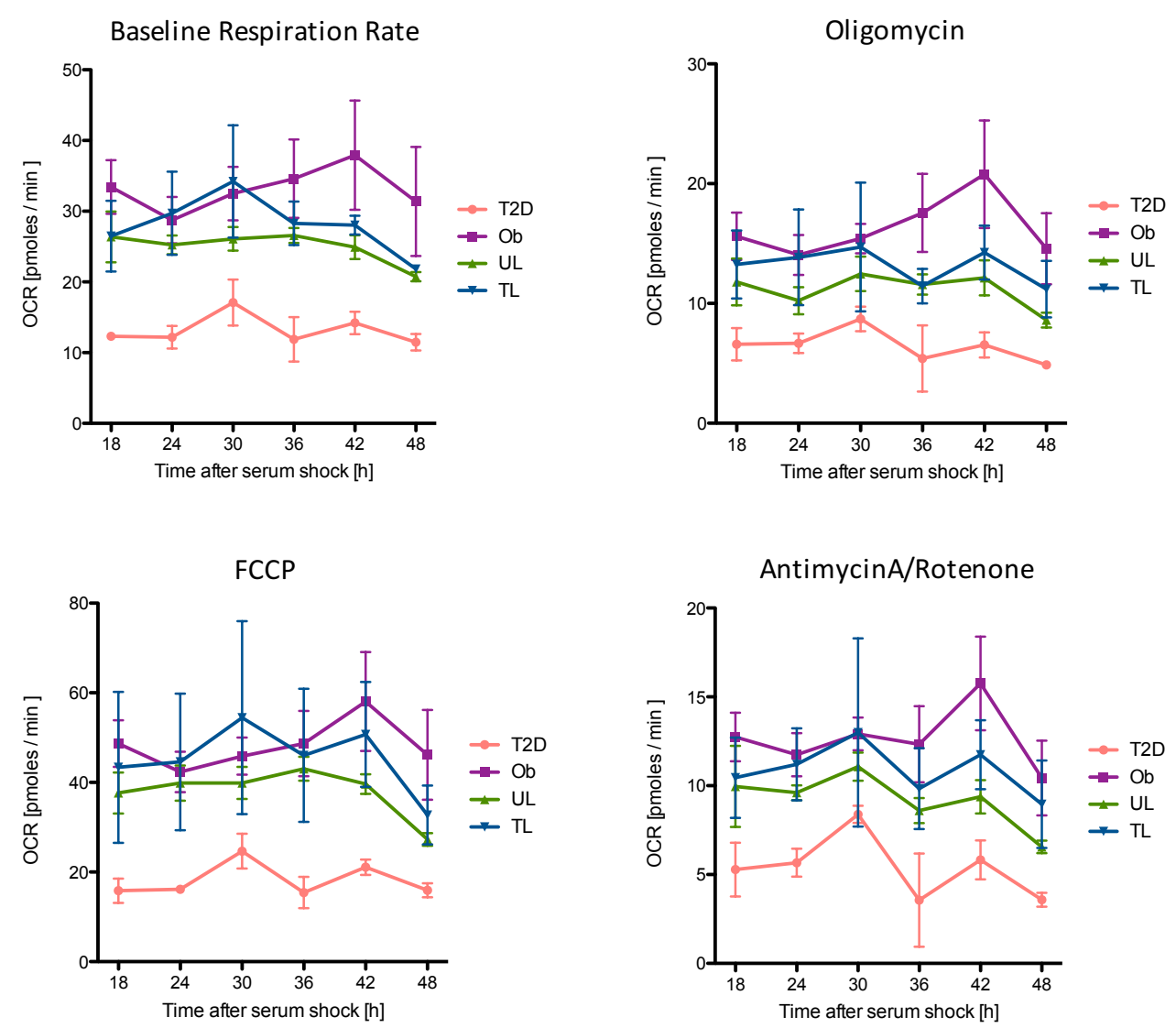

Figure 2: Raw cellular oxygen consumption rate (OCR):

A) baseline respiration rate B) oligomycin-induced mitochondrial uncoupling, C) FCCPinduced maximal respiration and D) non-mitochondrial respiration. $n=3$, error bars are $\pm S E M$

\section{Total protein content is rhythmic}

Mitochondrial respiration measurements were performed in cells that were differentiated in the seahorse 96 well plates. To normalize for putative differences in cell number, we determined total protein content and corrected OCRs for cellular protein content. Remarkably, total protein content showed significant oscillations over the 24-hour time period. Thus, two-way ANOVA showed a significant time $(p<0.001)$ and group $(P<0.001)$ effect for total protein content (Figure 4A), but no group*time interaction was observed. Protein content was then used to normalize mitochondrial respiration rates. 
Mitochondrial Basal Respiration Rate

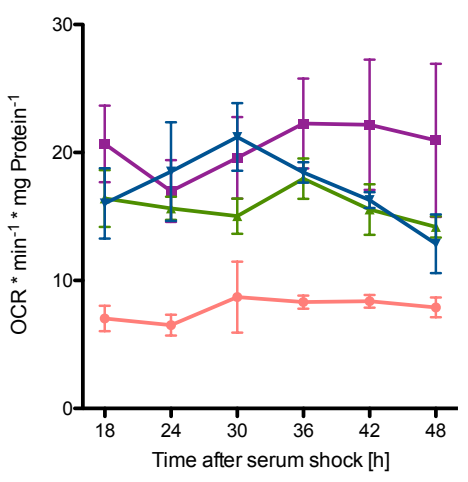

Maximal Respiration

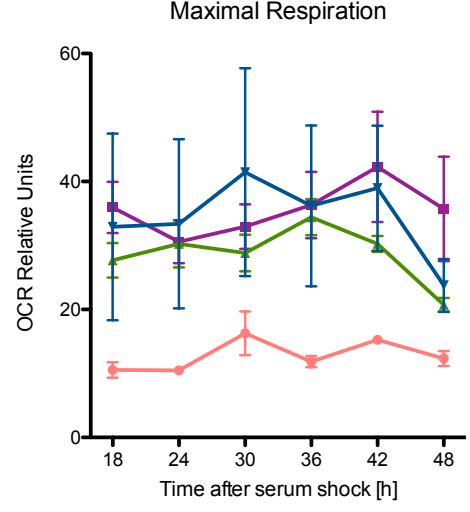

Coupled Respiration

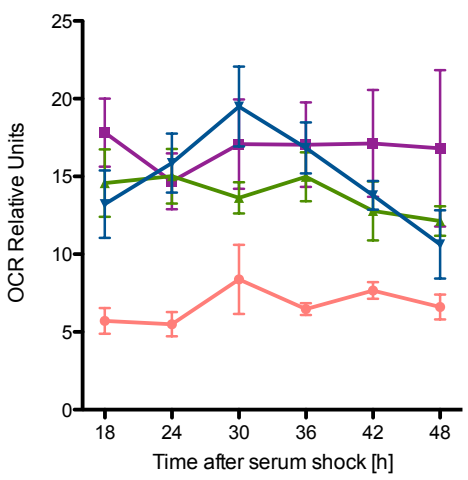

Proton Leak

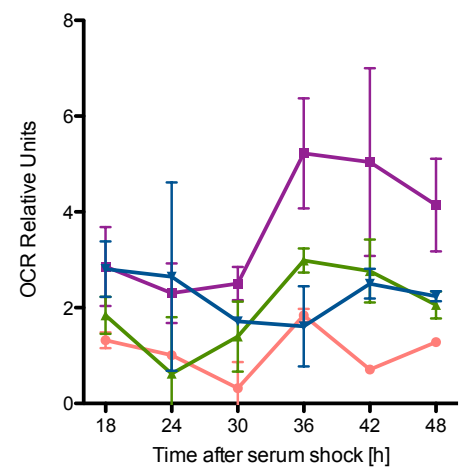

Mitochondrial Spare Capacity

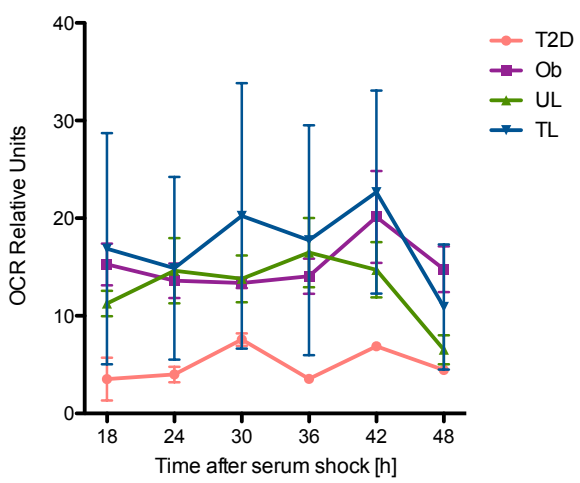

Figure 3: Parameters of mitochondrial metabolism

A) Mitochondrial basal respiration is determined by subtracting non-mitochondrial respiration from baseline oxygen consumption rate. B) Maximal mitochondrial respiration is determined by subtracting non-mitochondrial respiration from FCCP OCR. C) Mitochondrial spare capacity is determined by subtracting baseline OCR from FCCP OCR. D) Mitochondrial proton leak is determined by subtracting non-mitochondrial respiration from oligomycin OCR. E) Coupled respiration is determined by subtracting oligomycin OCR from baseline OCR. $n=3$, error bars are \pm SEM 
Upon protein correction two-way-ANOVA revealed significant time effects in mitochondrial respiration (B_OCR_Prot $p=0.003$, Oligo_OCR_Prot $p=0.003$, FCCP_OCR_Prot $p<0.001$,

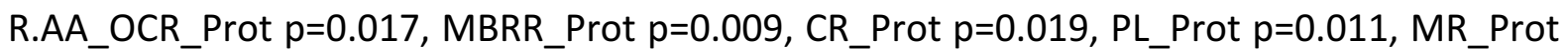
$p<0.001$, MSRC_Prot $p=0.012$ ), except for proton leak respiration ( $p=0.109)$. In addition, mitochondrial function normalized for cellular protein content remained lower in T2D, compared to TL, UL and OB (MBRR_Prot $p=0.008$, CR_Prot $p=0.025$, PL_Prot $p=0.009$, MR_Prot $p<0.001$, MSRC_Prot $p=0.011)$.

\section{Discussion}

Skeletal muscle is a major contributor to daily human energy metabolism. Recently, we showed in human muscle biopsies taken over a 24-hour period, that human skeletal muscle mitochondrial function possesses circadian rhythmicity [1]. In the current study, we examined if the rhythmicity in mitochondrial function is a cell autonomous characteristic of muscle that is retained in human primary myotubes. Our results showed that mitochondrial respiration in human primary myotubes did show rhythmicity, but only when mitochondrial respiration was normalized for muscle cell protein content, which in itself showed rhythmicity.

When analyzing whether mitochondrial function was affected by the metabolic phenotype of the donor, no difference in rhythmicity between groups were observed. However, as previously reported [5], we confirmed $\sim 50 \%$ lower mitochondrial respiration in human primary muscle cells derived from T2D donors in comparison to cellular respiration of TL, UL and $\mathrm{OB}$ donors. Interestingly, this attenuation in mitochondrial function was present at all time points and was reflected by both raw and protein-normalized oxygen consumption rates. This finding is in line with previous studies demonstrating that human primary myotubes retain their in vivo muscle phenotype in terms of lower mitochondrial lipid metabolism and reduced insulin sensitivity. Interestingly, in the current study, the reduced mitochondrial function in T2D remained also after the serum shock procedure, which sufficiently resynchronizes the muscle transcriptional-translational feedback loop as demonstrated previously [5]. Therefore, this finding indicates that human primary muscle cells resemble the functional phenotypical characteristics of the donor groups also throughout time. 
A

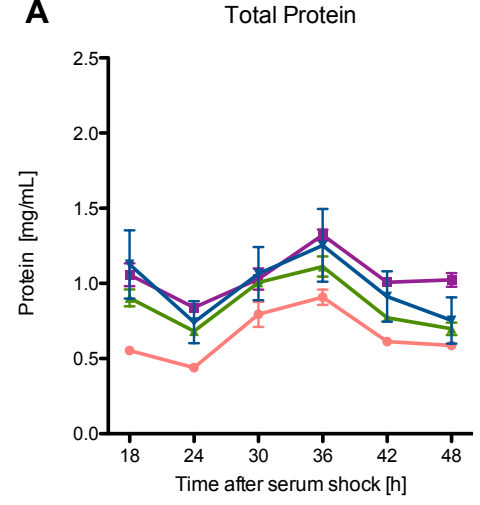

B

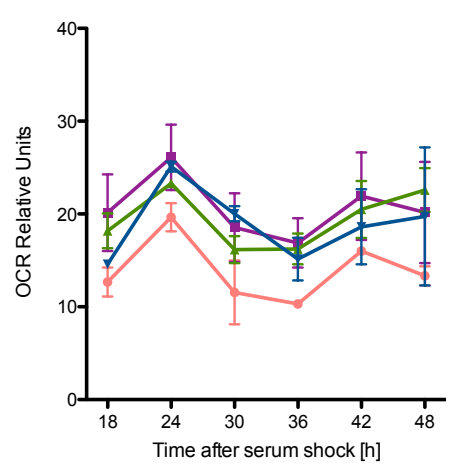

Coupled Respiration

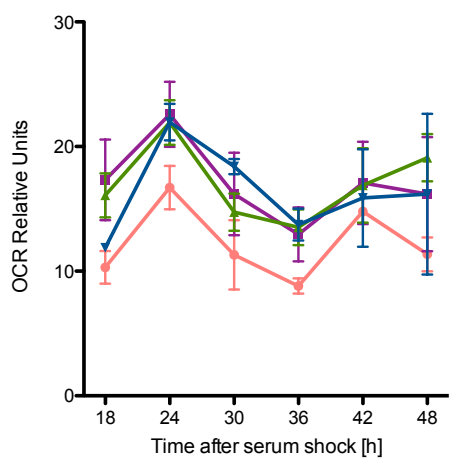

Mitochondrial Spare Capacity

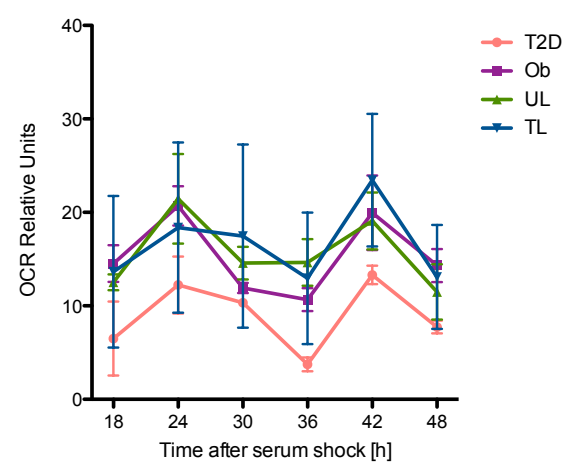

COSINOR fit of Total Protein

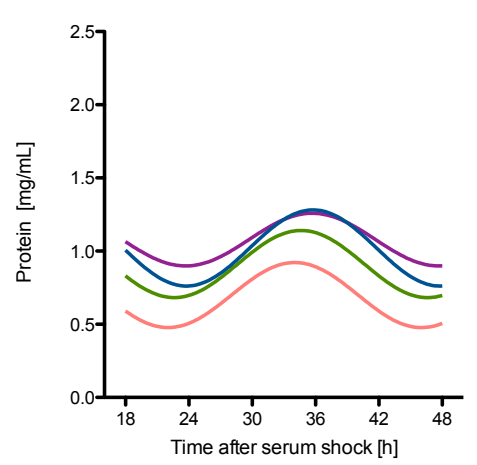

Maximal Respiration

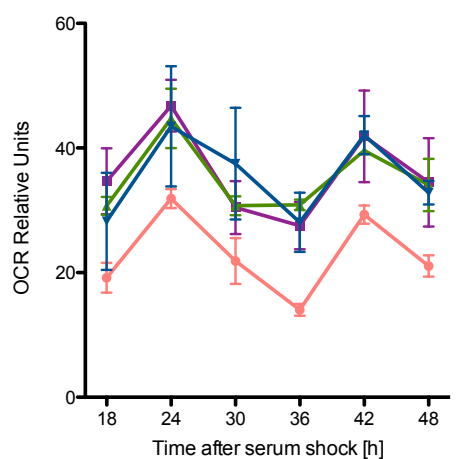

Proton Leak

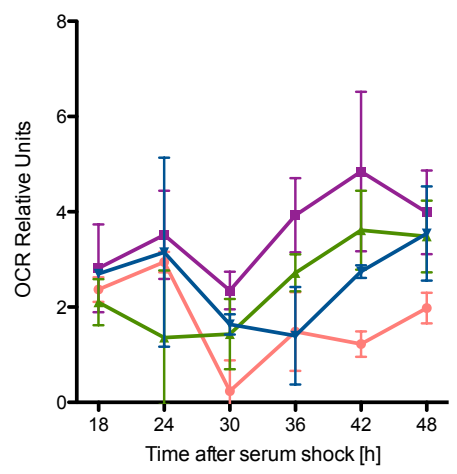

Figure 4: Circadian rhythmicity in total protein content and normalized respiration

A) total protein content with corresponding COSINOR fit, B) parameters of mitochondrial respiration normalized for total protein. 
Despite the albeit large inter-individual variance encountered within all four donor groups, we conclude that under synchronized but constant cell culture conditions, skeletal myotube mitochondrial respiration did not oscillate in an intrinsic circadian manner. The same conclusion could be made when assessing mitochondrial basal respiration rate (MBRR), coupled (CR) and uncoupled (PL) mitochondrial respiration as well as mitochondrial spare respiratory capacity (MSRC) and maximal respiration (MR). Again, none of these functional mitochondrial parameters were intrinsically rhythmic. However, since mitochondrial respiration measurements were made in 96-well plates, and differences in differentiation could potentially affect the results, we used total protein content as a proxy for cell number. When doing so in our current cell study, we unexpectedly found that total protein content itself showed rhythmicity. Although similar number of cells were seeded, total protein content (often used as a measure of total cell content) revealed robust rhythmic patterns with a fluctuation of approximately $50 \%$, in all four groups. These rhythmic patterns were aligned in phase between the groups and did not differ in amplitude. After correcting for protein content, mitochondrial respiration did show circadian rhythmicity, although it needs to be stressed that such circadian rhythm in mitochondrial function was completely dependent on protein normalization.

As this oscillation did not originate from mitochondrial respiration per se, it is difficult to conclude if mitochondrial respiration is truly rhythmic in human primary myotubes. Unfortunately, further elucidation of mitochondrial-DNA copy number and protein composition could not be pursued nor could the reason for the rhythmicity in total protein content be revealed. Hence, future studies should investigate whether overall protein content indeed changes over time or whether the observed rhythm in protein-normalized respiration could also be explained independently by rhythms in mitochondrial protein abundance or -activity, to result in a putative true rhythm of mitochondrial respiration. In that context, we previously reported that protein content of the mitochondrial complexes did not show rhythmicity, nor did mitochondrial-DNA copy [1]. It therefore seems unlikely that the rhythmicity in total protein content observed in the current study is of true translational value, and suggest that overall, mitochondrial function does not show circadian rhythmicity in myotubes cultured from human volunteers. 
Although this conclusion may not appear to be in line with our recent finding that human skeletal muscle displays a 24-hour rhythm in mitochondrial oxidative capacity, a major difference - by design - between our current cell study and the human study, is the absence of fasted/fed cycles or physical activity regimes. Since our intention was to investigate, whether such rhythmicity in mitochondrial is intrinsic to skeletal muscle cells, we needed to isolate those from physiological entraining time cues. Noteworthy, the skeletal muscle molecular clock drives circadian cellular processes $[5,9]$, but it has also been demonstrated elaborately, that in mice with a non-functional central clock fasted/fed cycles and physical activity regimes are sufficient to restore circadian clock rhythms in peripheral tissues such as skeletal muscle and liver [10-13]. These findings may suggest that behavioral cues such as food intake and physical activity are highly potent behavioral cues to adapt the muscle molecular clock. Given that under the constant and optimized culture conditions mitochondrial respiration did not show rhythmicity may suggest that the behavioral cues have a major impact on the circadian rhythmicity observed in our previous human study. However, the difference between models and the effect of serum shock on mitochondrial respiration cannot be ignored and further research is needed to address if in human mitochondrial respiration is truly intrinsically rhythmic.

In conclusion, our data demonstrates that human skeletal muscle cells do not display an intrinsic circadian rhythmicity in mitochondrial respiration, although $24 \mathrm{~h}$ variation in total cellular protein content precludes strong conclusion on the question if mitochondrial respiration may be affected by the molecular clock. The reason for the $24 \mathrm{~h}$ variation in total cellular protein content could not be revealed from the present study and needs future investigation. 


\section{References}

[1] van Moorsel, D., J. Hansen, B. Havekes, F.A. Scheer, J.A. Jorgensen, J. Hoeks, et al., 2016. Demonstration of a day-night rhythm in human skeletal muscle oxidative capacity. Mol Metab 5(8):635-645.

[2] Peek, C.B., A.H. Affinati, K.M. Ramsey, H.Y. Kuo, W. Yu, L.A. Sena, et al., 2013. Circadian clock NAD+ cycle drives mitochondrial oxidative metabolism in mice. Science 342(6158):1243417.

[3] Andrews, J.L., X. Zhang, J.J. McCarthy, E.L. McDearmon, T.A. Hornberger, B. Russell, et al., 2010. CLOCK and BMAL1 regulate MyoD and are necessary for maintenance of skeletal muscle phenotype and function. Proc Natl Acad Sci U S A 107(44):1909019095.

[4] Jacobi, D., S. Liu, K. Burkewitz, N. Kory, N.H. Knudsen, R.K. Alexander, et al., 2015. Hepatic Bmal1 Regulates Rhythmic Mitochondrial Dynamics and Promotes Metabolic Fitness. Cell Metab 22(4):709-720.

[5] Hansen, J., S. Timmers, E. Moonen-Kornips, H. Duez, B. Staels, M.K. Hesselink, et al., 2016. Synchronized human skeletal myotubes of lean, obese and type 2 diabetic patients maintain circadian oscillation of clock genes. Sci Rep 6(35047.

[6] Balsalobre, A., F. Damiola, and U. Schibler, 1998. A serum shock induces circadian gene expression in mammalian tissue culture cells. Cell 93(6):929-937.

[7] Sparks, L.M., M. Bosma, B. Brouwers, T. van de Weijer, L. Bilet, G. Schaart, et al., 2014. Reduced incorporation of fatty acids into triacylglycerol in myotubes from obese individuals with type 2 diabetes. Diabetes 63(5):1583-1593.

[8] Vosselman, M.J., J. Hoeks, B. Brans, H. Pallubinsky, E.B. Nascimento, A.A. van der Lans, et al., 2015. Low brown adipose tissue activity in endurance-trained compared with lean sedentary men. Int J Obes (Lond) 39(12):1696-1702.

[9] Perrin, L., U. Loizides-Mangold, S. Skarupelova, P. Pulimeno, S. Chanon, M. Robert, et al., 2015. Human skeletal myotubes display a cell-autonomous circadian clock implicated in basal myokine secretion. Mol Metab 4(11):834-845.

[10] Adamovich, Y., L. Rousso-Noori, Z. Zwighaft, A. Neufeld-Cohen, M. Golik, J. KrautCohen, et al., 2014. Circadian clocks and feeding time regulate the oscillations and levels of hepatic triglycerides. Cell Metab 19(2):319-330.

[11] Hara, R., K. Wan, H. Wakamatsu, R. Aida, T. Moriya, M. Akiyama, et al., 2001. Restricted feeding entrains liver clock without participation of the suprachiasmatic nucleus. Genes Cells 6(3):269-278.

[12] Feillet, C.A., U. Albrecht, and E. Challet, 2006. "Feeding time" for the brain: a matter of clocks. J Physiol Paris 100(5-6):252-260.

[13] Wolff, G. and K.A. Esser, 2012. Scheduled exercise phase shifts the circadian clock in skeletal muscle. Med Sci Sports Exerc 44(9):1663-1670. 


\section{CHAPTER 5}

\section{Demonstration of a day-night rhythm in human skeletal muscle oxidative capacity}

Published in Molecular Metabolism: 2016 1-11

Authors:

Dirk van Moorsel”, Jan Hansen”, Bas Havekes, Frank AJL. Scheer, Johanna A. Jörgensen , Joris Hoeks, Vera B. Schrauwen-Hinderling, Helene Duez, Philippe Lefebvre, Nicolaas C. Schaper, Matthijs K.C. Hesselink, Bart Staels , Patrick Schrauwen

$=$ These authors contributed equally. 


\begin{abstract}
Objective: A disturbed day-night rhythm is associated with metabolic perturbations that can lead to obesity and type 2 diabetes mellitus (T2DM). In skeletal muscle, a reduced oxidative capacity is also associated with the development of T2DM. However, whether oxidative capacity in skeletal muscle displays a day-night rhythm in humans has so far not been investigated.

Methods: Lean, healthy subjects were enrolled in a standardized living protocol with regular meals, physical activity and sleep to reflect our everyday lifestyle. Mitochondrial oxidative capacity was examined in skeletal muscle biopsies taken at five time points within a 24-hour period.

Results: Core body temperature was lower during the early night, confirming a normal daynight rhythm. Skeletal muscle oxidative capacity demonstrated a robust day-night rhythm, with a significant time effect in ADP-stimulated respiration (state $3 \mathrm{MO}$, state $3 \mathrm{MOG}$ and state 3MOGS, $\mathrm{p}<0.05$ ). Respiration was lowest at 1PM and highest at 11PM (state 3MOGS: $80.6 \pm 4.0$ vs. $95.8 \pm 4.7 \mathrm{pmol} / \mathrm{mg} / \mathrm{s}$ ). Interestingly, the fluctuation in mitochondrial function was also observed in whole-body energy expenditure, with peak energy expenditure at 11PM and lowest energy expenditure at 4AM $(p<0.001)$. In addition, we demonstrate rhythmicity in mRNA expression of molecular clock genes in human skeletal muscle. Conclusions: Our results suggest that the biological clock drives robust rhythms in human skeletal muscle oxidative metabolism. It is tempting to speculate that disruption of these rhythms contribute to the deterioration of metabolic health associated with circadian misalignment.
\end{abstract}




\section{Introduction}

Many metabolic processes are synchronized to day-night cycles by the circadian clock, thereby anticipating changes in metabolic activity associated with feeding or fasting and physical activity or rest [1]. In our modern "24/7" society, however, many individuals do not adhere to the lifestyle imposed upon us by nature. In this respect, epidemiological studies have shown that circadian misalignment - desynchronization between the intrinsic circadian and behavioral cycles, as is typical in shift-work - is associated with obesity, insulin resistance and type 2 diabetes mellitus (T2DM) [2-5]. Moreover, intervention studies have shown that challenging behavior by controlled circadian misalignment results in metabolic aberrations like decreased glucose tolerance and insulin sensitivity [6-8]. Circadian rhythms are governed by a central circadian clock, which is situated in the suprachiasmatic nucleus of the hypothalamus and is sensitive to light as the most important time cue (Zeitgeber) [9]. Interestingly, peripheral tissues have their own clocks. These peripheral clocks are synchronized by the central clock, but they can also be influenced by behavior, such as feeding or exercise $[10,11]$. The peripheral clock consists of transcriptional-translational feedback loops. The positive loop consists of the heterodimer of the CLOCK (circadian locomotor output cycles kaput) and BMAL1 (brain and muscle ARNT-like 1) proteins. The negative feedback loop is mediated via heterodimers of the proteins PER (Period) and CRY (Cryptochrome), which repress CLOCK/BMAL-1-controlled gene expression [9, 12]. Interestingly, in mouse and cell models, several components of the molecular clock have been causally linked to mitochondrial metabolism [13], mitochondrial integrity and density [14], mitochondrial dynamics [15] and metabolic flexibility [16]. So far however, it is unknown whether mitochondrial metabolism also displays a day-night rhythm in human skeletal muscle. Such data would be relevant, since reduced skeletal muscle oxidative capacity is associated with T2DM $[17,18]$. It is tempting to speculate that disturbances in day-night rhythm may affect muscle mitochondrial metabolism and thereby deteriorate metabolic health. Here, we investigated whether skeletal muscle mitochondrial function displays day-night rhythmicity by taking multiple muscle biopsies from healthy, lean volunteers within a $24 \mathrm{~h}$ period, under tightly controlled experimental conditions. To this end, we used a well-controlled research setting that reflects real life conditions, with regular meals, physical activity and a regular sleep/wake cycle. For this reason, we use the term 
day-night rhythm instead of circadian rhythm [19]. We here show that gene expression in muscle displays rhythmicity, which is specifically evident for the core components of the molecular clock. Furthermore, we are the first to show the presence of a day-night rhythm in human skeletal muscle oxidative capacity.

\section{Material and methods}

\section{Participants}

Twelve young lean male Caucasian individuals (age \pm SD: $22.2 \pm 2.3$ years, BMI \pm SD: $22.4 \pm$ $2.0 \mathrm{~kg} / \mathrm{m}^{2}$ ) participated in this study. The participants did not engage in exercise more than 3 hours per week, were non-smokers, had no active diseases and used no medication, verified by a medical questionnaire. Participants were selected for having a regular sleep duration (normally 7-9 hours/night), not having done shift work or having travelled across more than one time zone for at least 3 months. A morningness-eveningness questionnaire (MEQ-SA) was used to exclude extreme morning larks or night owls (MEQ-SA score mean \pm SD: $50 \pm 7$ ). All participants provided written informed consent. The study was approved by the Ethics Committee of the Maastricht University Medical Center, monitored by the Clinical Trial Center Maastricht and conducted in accordance with the principles of the declaration of Helsinki. All measurements were performed between November 2014 and July 2015. The study was registered at clinicaltrials.gov with identifier NCT02261168.

\section{Pre-study conditions}

One week prior to the study, participants were instructed to maintain a standardized lifestyle. This lifestyle included (trying to) sleep every night from 11PM until 7AM, eating breakfast, lunch and dinner at regular times (at 9AM, 2PM and 7PM) with no in-between snacks or drinks other than water. In this period subjects refrained from alcohol and caffeine. Participants were instructed not to exercise three days prior to the study. Two days before the study, we provided standardized meals (see below) to ensure standardized caloric and macronutrient intake for all participants. Lifestyle was monitored by accelerometry (activPAL3 physical activity monitor, PAL Technologies, Glasgow, UK) and food- and sleep-diaries, and checked at the start of the first study-day. 


\section{Study design}

Participants were admitted to the research unit at noon on study day 1 and stayed for 44 hours in total, under standardized conditions mimicking a real-life situation. The first studyday was mainly used to standardize and monitor meals, physical activity and bedtime. Meals were provided at fixed times (9AM, 2PM and 7PM). To prevent a sedentary lifestyle, participants went for a 15-minute, low-intensity walk accompanied by a researcher one hour after every meal. Directly hereafter, participants were instructed to stand for 15 minutes before they were allowed to sit again. In-between meals, physical activity and tests, the participants stayed in a respiration chamber; a small room with a bed, toilet, sink, desk, chair, TV and computer. During the first study-day we performed no measurements. At $11 \mathrm{PM}$, the lights of the respiration chamber were turned off and the participants were instructed to try to sleep. During this night, sleeping metabolic rate was measured by whole-room indirect calorimetry (Omnical, Maastricht Instruments, Maastricht, The Netherlands) (20).

The second study-day, participants were awakened at 6:30AM. Hereafter, participants swallowed a telemetric pill for measurement of core-body temperature. Next, an intravenous cannula was placed in the forearm for subsequent blood-draws. The first blooddraw was at 8AM, followed by an indirect calorimetry measurement using a ventilated hood while awake and at rest in supine posture to calculate resting energy expenditure and substrate oxidation. Directly hereafter, the first skeletal muscle biopsy was taken (described below). These measurements (blood draw, ventilated hood measurement and skeletal muscle biopsy) were repeated five times within 24 hours: at 8AM, 1PM, 6PM, 11PM and 4AM the next day. Additional blood samples were taken 2-hourly (10AM, 12PM, 2PM, 4PM, 8PM, 10PM, OAM, 2AM, 6AM and 8AM). The timing of meals and physical activity was similar to study-day 1 and subjects stayed within the respiration chamber in-between measurements. After the 11PM biopsy, participants went back to the respiration chamber to sleep with lights off. At 4AM, the participant was woken-up and the last measurements were performed, after which the subject was allowed to sleep until 7AM. After the 8AM blood draw the study protocol ended. 


\section{Study meals}

Two days before the study and during the study participants were provided with standardized meals, according to Dutch and US dietary guidelines. Caloric intake for consumption at home was calculated by multiplying the estimated resting metabolic rate, obtained with the Harris-Benedict formula [21] with an activity factor of 1.5. Participants were provided with optional extra snacks to eat with their meals if they were still hungry, up to an activity factor of 1.7. For the first study-day in the laboratory, energy requirement was calculated by multiplying the estimated resting metabolic rate with an activity factor of 1.35 , because of limited physical activity in the research facility. For the second study-day, energy requirement was calculated by multiplying the sleeping metabolic rate of the first study night (measured by whole-room indirect-calorimetry) by 1.5. During the study days, participants received 3 meals daily. Breakfast accounted for $\sim 21$ energy\%, lunch for $\sim 30$ energy\% and dinner for $\sim 49$ energy\%. Daily macronutrient composition was $\sim 52$ energy\% as carbohydrates, $\sim 31$ energy\% as fat ( $\sim 9 \%$ saturated) and $\sim 14$ energy\% as protein. No snacks or drinks other than water were provided in-between meals.

\section{Skeletal muscle biopsies and respirometry}

Five skeletal muscle biopsies were obtained from the $\mathrm{m}$. vastus lateralis according to the Bergström method [22] under local anesthesia (1\% lidocaine, without epinephrine). Each biopsy was taken from a separate incision at least $2 \mathrm{~cm}$ from the previous incision, moving from distal to proximal. The first biopsy was randomly taken from the left or right leg, and each subsequent biopsy was taken from the other leg. Part of the biopsy was immediately placed in ice-cold preservation medium (BIOPS, OROBOROS Instruments, Innsbruck, Austria) and used for measurement of mitochondrial oxidative capacity. For this analysis, intact muscle fibers were permeabilized and measured for oxygen consumption upon several substrates using an Oxygraph (OROBOROS Instruments). During the assay, muscle fibers were supplemented with malate, octanoylcarnitine, ADP, glutamate, succinate and carbonylcyanide p-trifluoromethoxyphenylhydrazone (FCCP) as described previously [23]. The remaining part of the muscle biopsy was immediately frozen in melting isopentane and stored in $-80^{\circ} \mathrm{C}$ until further analysis. 


\section{Indirect calorimetry}

To calculate whole-body energy expenditure, respiratory exchange ratio (RER), glucose and fat-oxidation, oxygen consumption and carbon dioxide production were measured with an automated respiratory gas analyzer using a ventilated hood system (Omnical; IDEE, Maastricht, the Netherlands). Calculations of energy expenditure and substrate oxidation were made with the assumption of a negligible protein oxidation [24, 25].

\section{Measurement of core-body temperature}

Core-body temperature was measured by a swallowed telemetric pill (CorTemp HT150002; $\mathrm{HQ}$ Inc.). The telemetric pill measured the core-body temperature every 10 seconds and sent the information wirelessly to a portable receiver worn outside the body on the stomach. For further analysis, we used temperature averages of every 10 minutes, after excluding false measurements.

\section{Gene transcript quantification:}

RNA was isolated from $50 \mathrm{mg}$ of muscle material by TRIzol lysis (Qiagen, Hilden, Germany). RNA was further purified by the RNeasy kit from Qiagen (Hilden, Germany).

Microarray analysis: RNA integrity was assessed using a Bioanalyzer 2100 (Agilent) and RIN were above 9.0. RNAs were then processed to generate labeled SSDNA using the Whole Transcript cDNA synthesis and amplification and the Terminal Labeling kit as suggested by the manufacturer (Affymetrix). Samples were hybridized to HuGene 2.0 arrays and native .CEL files imported into GeneSpring (v13.1.1, Agilent) after quality controls using the Affymetrix Expression Console. The RMA16 algorithm was used for summarization and data was normalized to the median of all samples. Normalized intensity values were used to generate a self-organized map (distance metric: Euclidean; number of iterations: 500). Protein-encoding genes from specific clusters were searched against the GO Biological Process, KEGG and Reactome databases using the Metascape tool [26]. Microarray data have been deposited in NCBls Gene Expression Omnibus (GEO, http://www.ncbi.nlm.nih.gov/geo/) and are accessible through GEO accession number GSE79934.

RT-QPCR: RNA integrity was assessed using a Bioanalyzer (Agilent Technologies, Santa Clara, USA) and yield was measured using a NanoDrop spectrophotometer (Thermo Fisher 
Scientific, Waltham, USA). The high-capacity RNA-to-cDNA kit from Applied Biosystems (Foster City, USA) was used for transcribing $0.5 \mu \mathrm{g}$ RNA to cDNA. Transcript abundance was determined using a 7900HT Fast Real-Time PCR System (Applied Biosystems, Waltham, USA). To minimize the variability in reference gene normalization, the geometric mean of three reference genes (RPL26, GUSB and CYPB), which were individually stably expressed in time, was used. This geometric mean was used as the internal reference for comparative gene expression analysis in the remainder of the study [27].

\section{Protein analysis}

Western blot analyses were performed in RIPA-lysates of human muscle tissue. Protein concentration was determined using the Bio-Rad RC/DC kit (Bio-Rad Laboratories, Veenendaal, The Netherlands). Equal amounts of protein were loaded on 12\% TGX gels (BioRad Laboratories) or 4-12\% Bolt gradient gels (Novex, Thermo Fisher Scientific, Bleiswijk, The Netherlands). Proteins were transferred to nitrocellulose with the Trans-Blot Turbo transfer system (Bio-Rad Laboratories). Primary antibodies: a cocktail of mouse monoclonal antibodies directed against human OXPHOS (dilution 1:10,000; ab110411, Abcam, Cambridge, UK), two mitochondrial markers directed against TOMM20 (dilution 1:10,000; ab186734; Abcam), porin/VDAC (dilution 1:10,000; sc-8828, Santa Cruz Biotechnology, Dallas, Texas), SR-actin (dilution 1:5,000; A-2172; Sigma Aldrich, Zwijndrecht, The Netherlands), PGC-1 (dilution 1:10,000, 516557, Calbiochem), FIS-1 (dilution 1:1000, sc98900, Santa Cruz Biotechnology, Dallas, Texas), PINK-1 (dilution 1:2000, sc-33796, Santa Cruz Biotechnology, Dallas, Texas) and OPA-1 (dilution 1:2500, 612606, Becton Dickinson). The specific proteins were detected using secondary antibodies conjugated with IRDye680 or IRDye800, and were quantified with the CLx Odyssey Near Infrared Imager (Li-COR, Westburg, Leusden, The Netherlands).

\section{Statistics}

Data are presented as mean \pm SEM (standard error of the mean) unless indicated otherwise. Statistical analyses were performed with the use of IBM Statistical Package for Social Sciences for MAC, version 23 (SPSS, Inc.). The effect of time on outcome variables was analyzed by repeated measures ANOVA. If the assumption of sphericity was violated (Mauchly's test), we applied Greenhouse-Geisser's correction. In case repeated measures 
ANOVA revealed significant effects for the oxidative capacity and indirect calorimetry analyses, Bonferroni adjusted post-hoc analyses were applied to look at significant differences between specific time-points. Statistical significance was defined as a p-value < 0.05. In addition, if repeated measures ANOVA resulted in a significant effect of time for targets of mRNA and protein expression and oxidative capacity states, we tested for rhythmicity using the JTK_CYCLE package in R 3.2.1 with Windows 8 [28]. For this analysis, values were normalized to subject mean prior to analysis.

\section{Results and discussion}

Participants adhered to a controlled normal lifestyle and displayed a normal day-night rhythm in core-body temperature. To investigate whether mitochondrial oxidative capacity displays a day-night rhythm, we performed an observational study in which twelve young lean male volunteers were admitted to our metabolic research unit for two days under controlled conditions, mimicking our normal daily life. The study protocol is graphically depicted in figure 1. Prior to the study days, subjects were instructed to adhere to a standardized lifestyle, including fixed sleeping times, which was monitored by accelerometry. During the second study-day we recorded core-body temperature to examine the presence of a normal day-night rhythm (supplemental figure 1). Indeed, all subjects showed a characteristic day-night pattern in core-body temperature with highest temperature at the end of the day and a decrease in temperature after midnight [29], indicating that chronotypes were similar across participants.

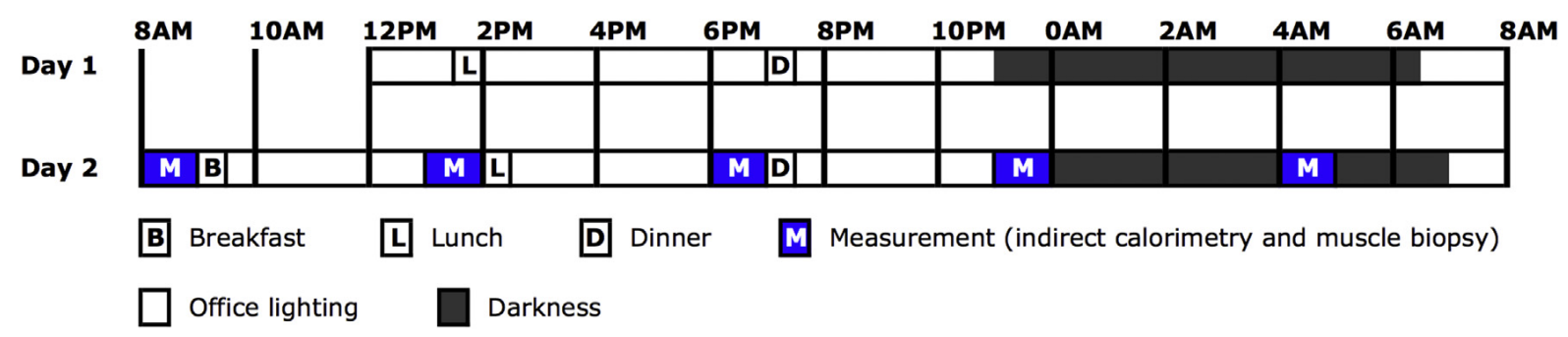

\section{Figure 1: Study design.}

Participants stayed in the research facility for 44 hours starting at noon of day 1. During the second study-day we performed all measurements. B, breakfast; $L$, lunch; $D$, dinner; $M$, measurement (indirect calorimetry and muscle biopsy). 
A

\section{$\sum_{\infty} \sum_{0} \sum_{0} \frac{\sum}{0} \frac{\sum}{\sigma}$}

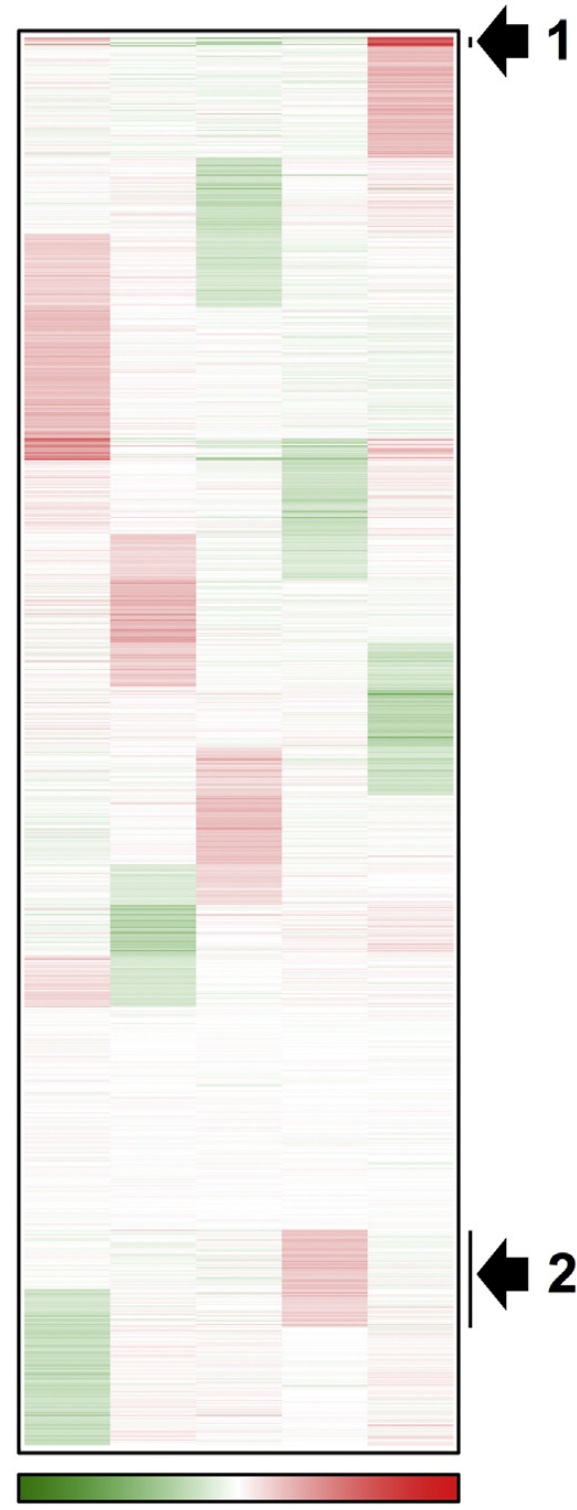

$-1,4 \quad \log _{2}$ FC $\quad 1,4$
B 4AM

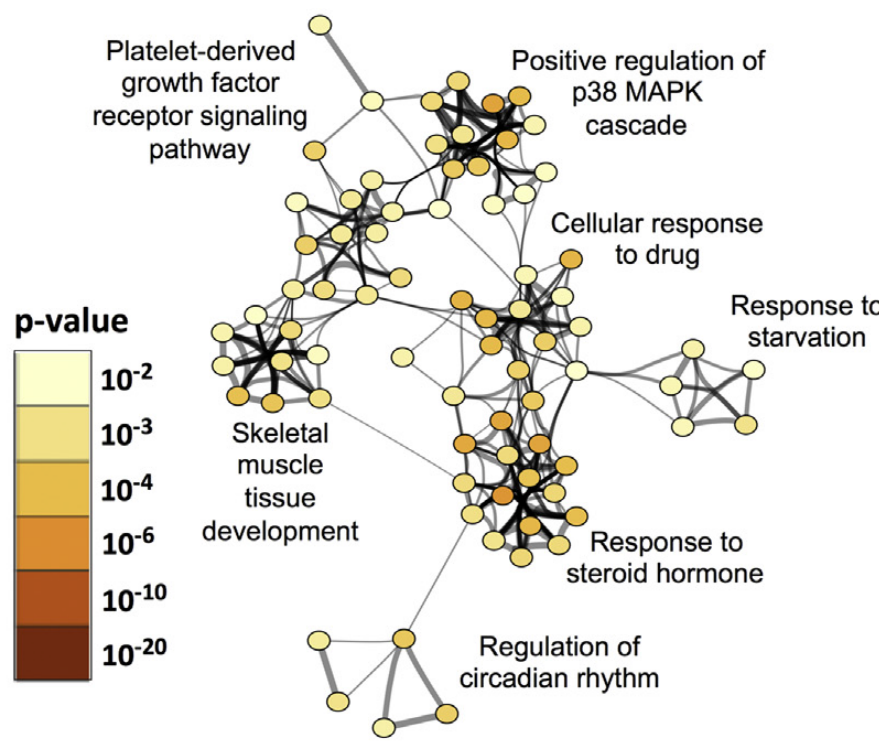

C 11PM
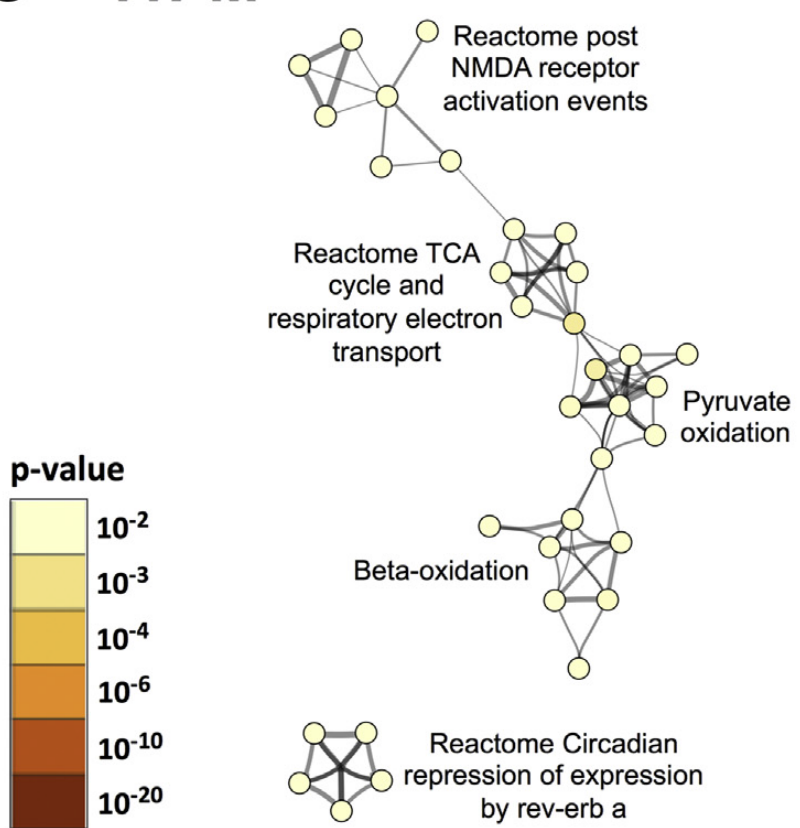

Figure 2: Time-dependent expression of transcripts in human skeletal muscle.

(A) Time-dependent gene expression was assessed by microarray analysis of RNA extracted from a single donor (N . 1). Protein-encoding transcript expression patterns were visualized as a self-organizing map (Euclidean distance metrics). The color scale indicates upregulated genes (red) and downregulated genes (green) relative to the global median signal of each array. The arrow indicates clusters of genes showing the most prominent peak of expression 
at the indicated time point (4 AM, cluster 1 and 11 PM, cluster 2). (B and C) Gene annotation enrichment analysis. The genes extracted from cluster 1 and 2 were searched using multiple databases (GeneOntology Biological Processes, KEGG pathways and Reactome) and statistically enriched terms were determined using the Metascape tool. The most significantly enriched terms are indicated for cluster 1 (GO biological processes, panel B, peak expression 4 AM) and cluster 2 (Reactome, panel C, peak expression 11 PM). Statistically significant terms were hierarchically clustered and converted into a network. Each term is represented by a circle node, of which the size is proportional to the number of genes in the term. The color of the node indicates the statistical significance of the term belonging to the cluster (see color scale). The most significant term characterizing each cluster is indicated. Similarities between terms are indicated by connecting lines.

A

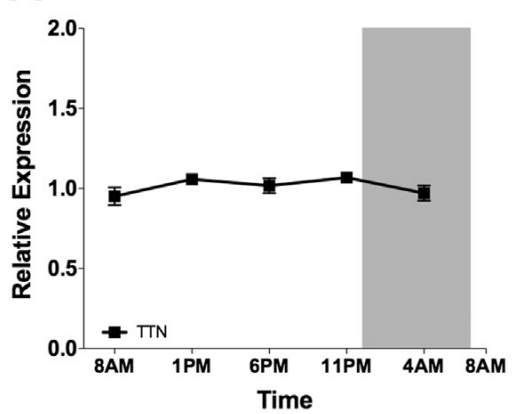

B

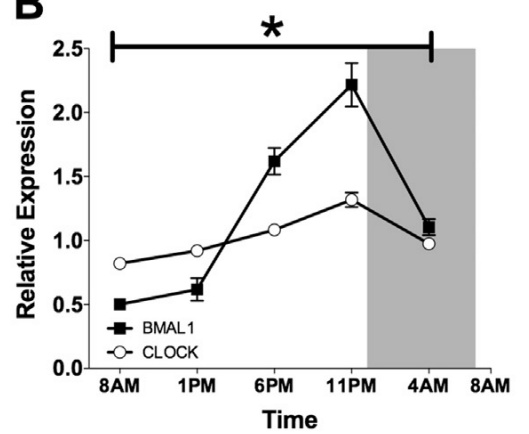

C

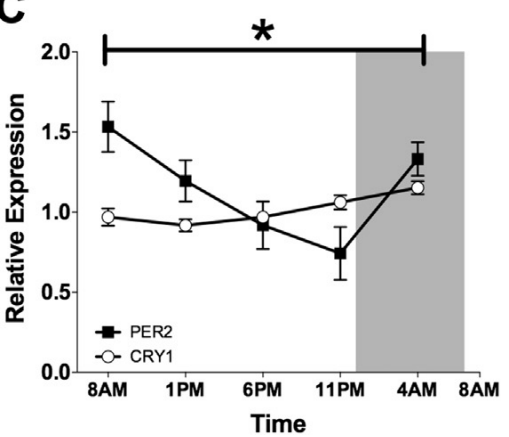

Figure 3: Rhythmicity of core molecular clock genes in human skeletal muscle.

Diurnal mRNA expression of the arrhythmic muscle filament gene TTN (A), the core clock genes CLOCK and BMAL1 (B), PER2 and CRY1 (C), measured by RT-QPCR. Data are normalized to the geometric mean of 3 housekeeping genes and presented as mean \pm SEM. ${ }^{*} p<0.05$ for the effect of time in all depicted genes.

Human skeletal muscle displays oscillations in RNA of metabolic and core molecular clock genes

During the second study day, we performed five consecutive skeletal muscle biopsies. To prevent meal interference, the biopsies were taken immediately before the three meals 
(8AM, 1PM, 6PM), before bedtime (11PM) and one time during the night (4AM) after waking up the participant.

To examine if skeletal muscle displays rhythmicity in gene expression, we analyzed RNA using DNA microarrays in subsequent biopsies from a single donor with a representative rhythm in mitochondrial oxidative capacity. This analysis revealed that $14.5 \%$ of transcripts (4094 out of 28168) displayed a cyclic expression peaking at different times, $75 \%$ of them corresponding to protein-encoding transcripts (figure 2A). Term enrichment against Gene Ontology, KEGG and Reactome databases indicated that gene clusters displaying a maximal expression in the late evening or during the night (11PM, 4AM) are notably related to the core clock machinery (4AM, figure 2B) including REV-ERB- $\alpha$, REV-ERB- $\beta$, PER3 and DBP and to the TCA cycle/respiratory electron transport chain (11PM, figure $2 \mathrm{C}$ ) such as components of NADH dehydrogenase complexes (NDUFA4, NDUFA8) and of ATP synthase (ATP5F1, ATP5G3, APTP5A1, ATP5L). Collectively, these analyses suggested the existence of a functional core clock machinery and oscillations in mitochondrial metabolism in human skeletal muscle.

Expression of core clock genes was validated in all participants by RT-QPCR assays. TTN mRNA expression, measured as an arrhythmic muscle filament gene, did not vary throughout the 24-hour period ( $p=0.331$, figure $3 A$ ). CLOCK mRNA showed significant variation over time (ANOVA $p<0.001$ ), but rhythmicity was of rather low amplitude (JTK_CYCLE $p<0.001$; figure 3B), consistent with previous observations in several mouse tissues [30]. BMAL1 mRNA exhibited a robust sinusoidal rhythm, with highest expression around midnight, and lowest expression in the morning and early afternoon (ANOVA $p<0.001$, JTK_CYCLE $p<0.001$; figure 3B). Of the negative feedback loop, PER2 was most abundantly expressed in the early morning ( $p=0.019$, figure $3 C$ ). CRY1 was most highly expressed in the night, however the amplitude was rather low ( $p=0.006$, figure $3 C)$. Both were confirmed to be rhythmic by JTK_CYCLE $(p<0.001)$.

Together, our data show robust rhythmicity of the molecular clock in human skeletal muscle, which is consistent with the demonstration of an oscillating molecular clock in other human cells and tissues like leukocytes [31], follicle cells [32] and adipose tissue [33] and in primary cultures of human muscle [34]. In addition, micro-array analysis in sequential biopsies obtained from a single donor revealed cyclic expression of several clusters of genes, including genes involved in important mitochondrial pathways. A previous study 
investigating the molecular clock in human adipose tissue biopsies demonstrated peak expression of BMAL1 at the very end of the light-phase, similar to our current results in human skeletal muscle [33]. Interestingly, that study examined adipose tissue gene expression under a standardized circadian rhythm protocol with subjects in the resting condition and with hourly nutritional drinks, thereby limiting the influence of behavior on the core molecular clock expression. The similarity in results suggests that the molecular clock patterns we here observe in skeletal muscle may indeed be dominated by circadian rhythmicity. This is further underscored by findings in animals, as in mouse skeletal muscle [35] and mouse liver [13, 15], BMAL1 MRNA is highest at the end of the dark-phase and beginning of the light phase. Since mice are nocturnal animals, this corresponds with a highest BMAL1 expression at the end of the active phase and beginning of the rest phase, similar to our findings in human skeletal muscle.

\section{Human skeletal muscle oxidative capacity displays a day-night rhythm}

To investigate whether the rhythmicity in mitochondrial and molecular clock genes was also reflected in day-night rhythms of muscle mitochondrial oxidative capacity, we performed high-resolution respirometry in freshly isolated permeabilized muscle fibers. Skeletal muscle oxidative capacity demonstrated a clear day-night rhythm, with a significant time effect in ADP-stimulated (state 3 ) respiration fuelled by different substrates (state $3 \mathrm{MO}$, state 3 MOG and state 3 MOGS $p=0.042 ; 0.016 ; 0.042$ respectively, figure 4A). Mitochondrial state 3 respiration was lowest at 1PM, and highest at 11PM (state 3 MOGS: $80.6 \pm 14.0$ vs. $95.8 \pm$ $16.3 \mathrm{pmol} / \mathrm{mg} / \mathrm{sec}$, mean $\pm \mathrm{SD}$ ). Differences between these timepoints were statistically significant for state $3 \mathrm{MO}(\mathrm{p}=0.032)$ and state 3 MOG $(p=0.027)$, although it just did not reach significance for state 3 MOGS $(p=0.132)$ after Bonferroni adjustment for multiple testing. Furthermore, JTK_CYCLE analysis of mitochondrial respiration confirmed rhythmicity for state $3 \mathrm{MO}(\mathrm{p}=0.007), 3 \mathrm{MOG}(\mathrm{p}=0.039)$ and 3 MOGS $(0.041)$. 

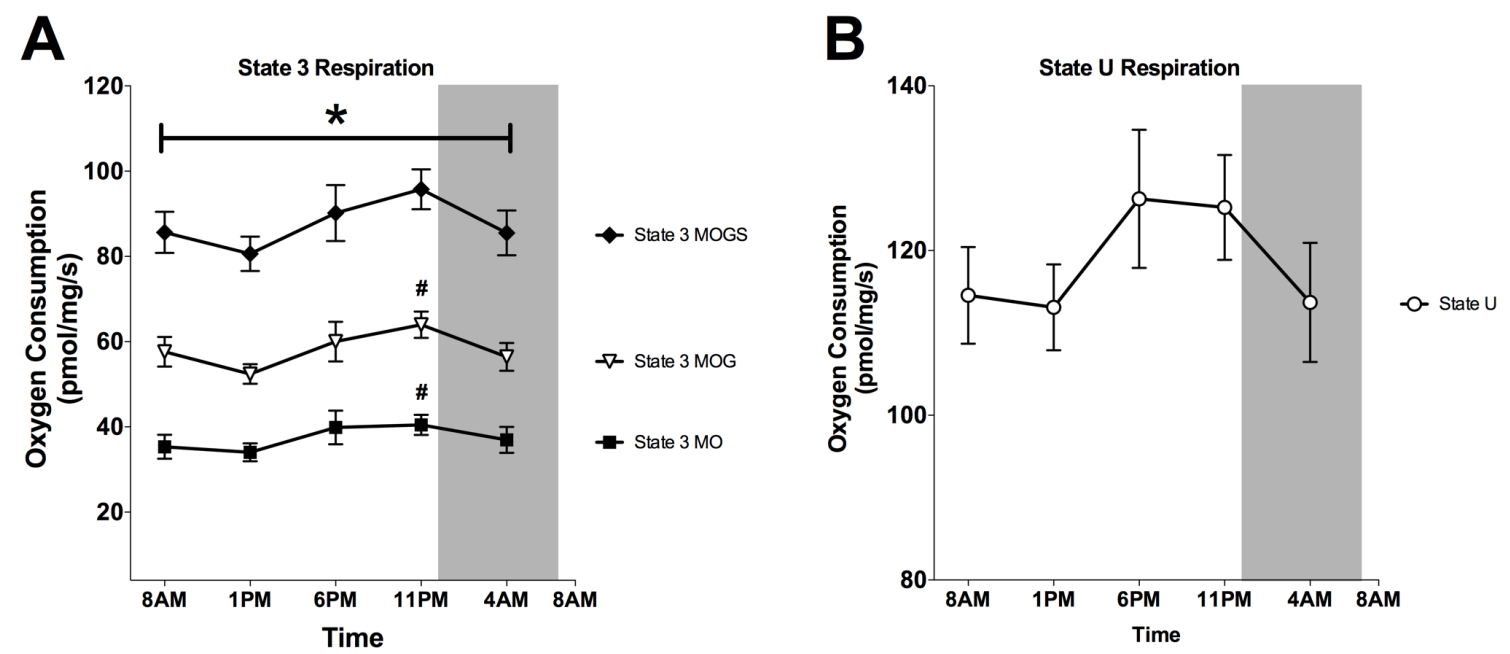

Figure 4: Mitochondrial oxidative capacity in human skeletal muscle displays a day-night rhythm.

(A) ADP-stimulated respiration of permeabilized muscle fibers upon a lipid substrate (state 3 MO); fuelled by complex I linked substrates (state 3 MOG) and upon parallel electron input into complex I and II (state 3 MOGS). (B) Maximally uncoupled respiration upon FCCP (State $U)$. $M$, malate; $O$, octanoylcarnitine; $G$, glutamate; $S$, succinate. Data represents oxygen consumption per mg wet weight per second and is depicted as mean \pm SEM. ${ }^{*} p<0.05$ for the effect of time in all states. \#p<0.05 vs 1PM for Bonferroni-adjusted post-hoc analysis.

Maximally uncoupled respiration, reflecting the maximal capacity of the electron transport system, demonstrated similar oscillations although the effect of time did not reach significance ( $p=0.121$, figure $4 B$ ). It is interesting to note that the difference in mitochondrial state 3 respiration between 1PM and 11PM was on average $20 \%$, which is in the same range as the difference in mitochondrial function between patients with T2DM and BMI-matched controls $[17,36]$, indicating that the magnitude of the day-night rhythm in mitochondrial function is of physiological relevance. Previous studies in animal models of metabolic diseases do show disrupted circadian rhythmicity in molecular clock of metabolic tissues and in plasma metabolites $[37,38]$, and it is therefore tempting to speculate that day-night rhythmicity in muscle mitochondrial metabolism is altered in T2DM and prediabetic patients. Such disturbances in circadian rhythmicity of mitochondrial function may contribute to reduced metabolic flexibility that is observed in metabolically compromised 
individuals and thereby contribute to the development of metabolic diseases, consistent with findings that circadian misalignment is associated with obesity and T2DM [2-4]. If this is indeed the case, restoring circadian rhythmicity may comprise a potential new basis for lifestyle advice and provide treatment options for T2DM.

How mitochondrial function can show circadian rhythmicity cannot be completely revealed from the current study in humans. However, to investigate if the rhythm in mitochondrial respiration is caused by intrinsic variation in mitochondrial oxidative capacity, rather than by variation in mitochondrial density, we measured several markers of mitochondrial content. Mitochondrial-DNA copy number - the ratio between the DNA copy number of a mitochondrial (ND1) and a nuclear gene (LPL) - did not reveal a day-night rhythm $(p=0.437$, figure 5A). Also, western blot analysis of structural subunits of the five mitochondrial respiratory chain complexes did not reveal a statistically significant effect of time (figure 5BG). In addition, the mitochondrial outer membrane proteins VDAC and TOMM-20 did not display day-night rhythmicity (figure $\mathbf{5 H}-\mathbf{I}$ ). The non-mitochondrial protein SR-Actin was also stable throughout the day (figure 5J). Together, these results suggest that in skeletal muscle, intrinsic mitochondrial function (rather than mitochondrial content) displays circadian rhythmicity.

We next investigated whether mitochondrial dynamics or mitochondrial biogenesis could play a role in the circadian rhythmicity of intrinsic mitochondrial function. Mitochondrial fission and fusion occur as a result of changes in energy demand, influence substrate metabolism and are controlled by the circadian clock in mouse liver [15, 39]. Here, we quantified proteins involved in mitochondrial biogenesis and mitochondrial dynamics in skeletal muscle biopsies. PGC-1 $\alpha$, the master regulator of mitochondrial biogenesis, did not show a significant difference over time $(p=0.901$, figure 6A). Interestingly however, FIS1, a marker of mitochondrial fission, displayed significant time-differences, with a pattern matching the rhythm in mitochondrial oxidative capacity ( $p=0.016$, figure $6 B$ ), although JTK_CYCLE analysis did not confirm significant rhythmicity.

PINK-1, a marker of mitophagy, follows a rhythm opposing FIS-1, although this time-effect just did not reach statistical significance ( $p=0.061$, figure $6 C)$. Noteworthy, in murine liver FIS-1 and PINK-1 24-hour expression patterns are also opposed [15]. 
A

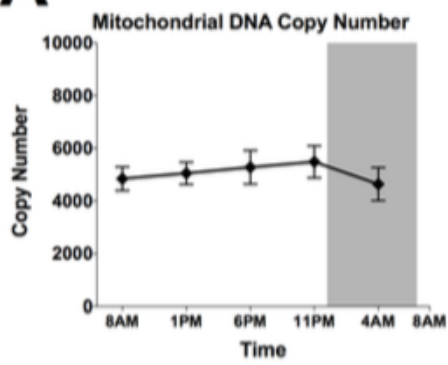

C
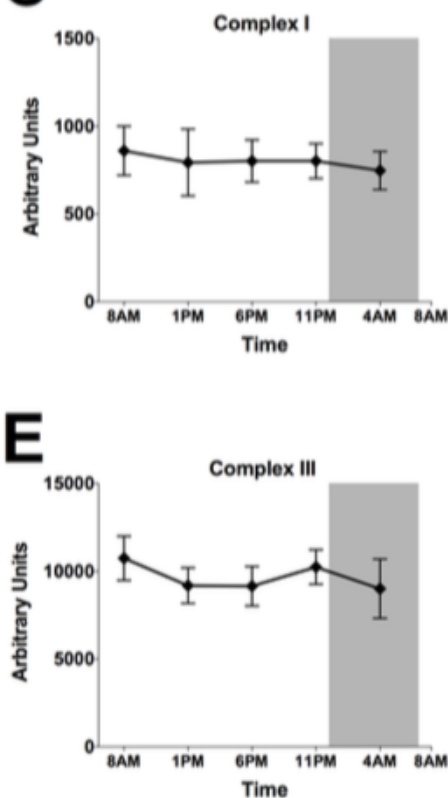

G

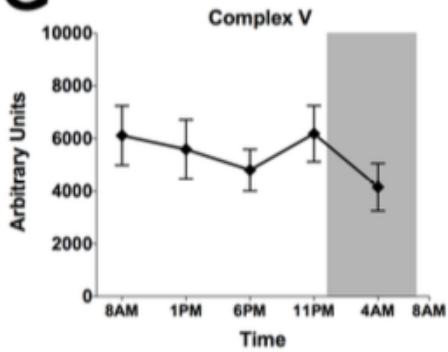

I
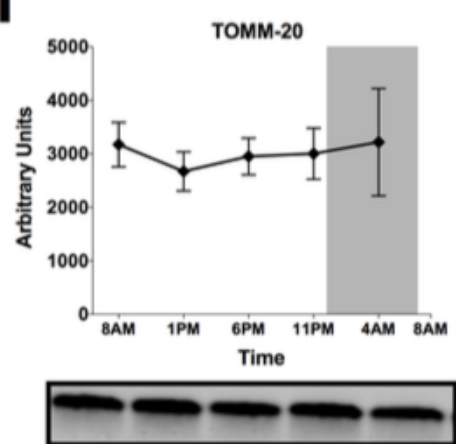

B

Oxidative Phosphorylation Complexes

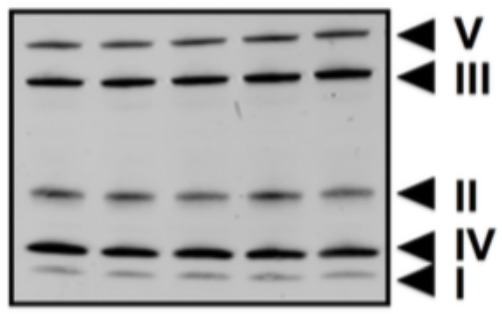

D

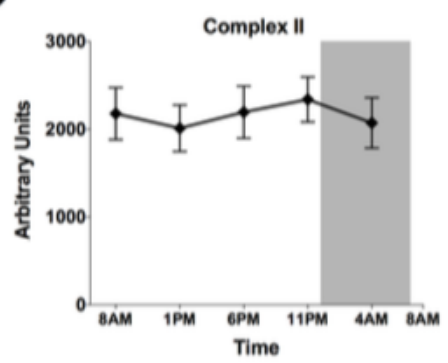

$\mathbf{F}$

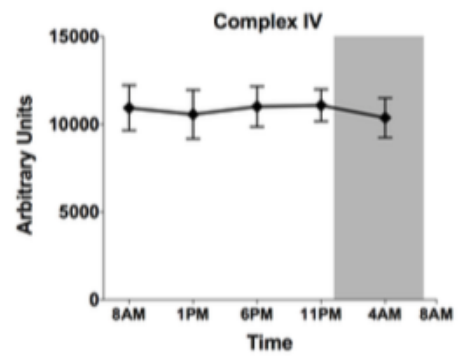

H
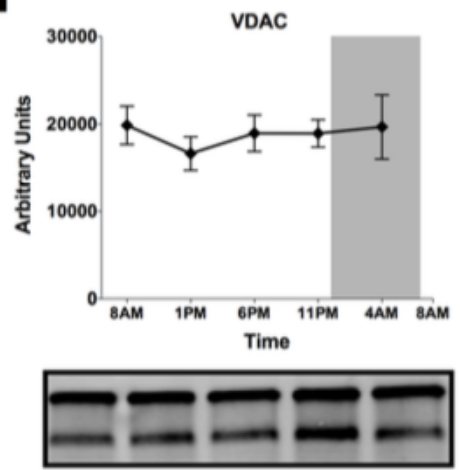

J

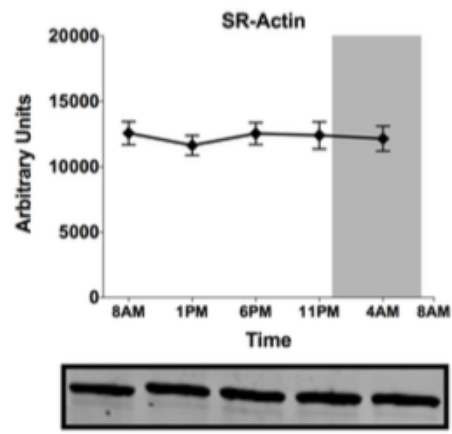


Figure 5: Mitochondrial content and mitochondrial marker proteins do not show rhythmicity.

(A) Measurement of mitochondrial DNA copy number (DNA copy numbers of the mitochondrial-encoded gene ND1 divided over the nuclear encoded gene LPL). (B) Representative western blot depicting the oxidative phosphorylation complexes of all timepoints (C-G) Protein levels of specific subunits of complex I-V of the mitochondrial electron transport chain, measured by western blotting. (G-I) Protein levels of other mitochondrial (VDAC, TOMM-20) and non-mitochondrial (SR-Actin) proteins measured by western blotting. Representative western blot images are displayed below their respective graphs. Data expressed as mean \pm SEM. $p>05$ for the effect of time in all panels.

Furthermore, the mitochondrial fusion marker OPA-1 showed a significant decrease over time, across all time points ( $p=0.045$, figure 6D, without significant rhythmicity in JTK_CYCLE analysis). These results suggest that mitochondrial dynamics may be involved in governing mitochondrial capacity around the clock, although mechanistic studies in preclinical models are needed to further investigate underlying mechanisms.

The physiological relevance of the rhythmicity in oxidative capacity cannot be deduced from the current study. It is, however, interesting to note that aerobic performance also peaks later in the day $[40,41]$. Our results could therefore form a physiological explanation for these findings. An earlier study found that chronotype influences the time-point of peak performance [41]. In our study, we only included participants with an average chronotype. Future studies will be needed to investigate whether early and late chronotypes have a different time-point of peak mitochondrial function.

\section{Whole-body energy expenditure peaks before midnight}

We next examined if day-night rhythmicity in muscle mitochondrial function was also associated with rhythmicity in whole-body measures of energy metabolism. To this end, we performed indirect calorimetry analyses to determine resting energy expenditure and substrate oxidation at the same time points of the muscle biopsy. Interestingly, similar to mitochondrial respiratory capacity, resting energy expenditure showed a significant time 
effect, with highest energy expenditure at 11PM ( $p<0.001$ for the time-effect; $p=0.005$ for Bonferroni adjusted post-hoc analysis 11PM vs 4AM; figure 7A).
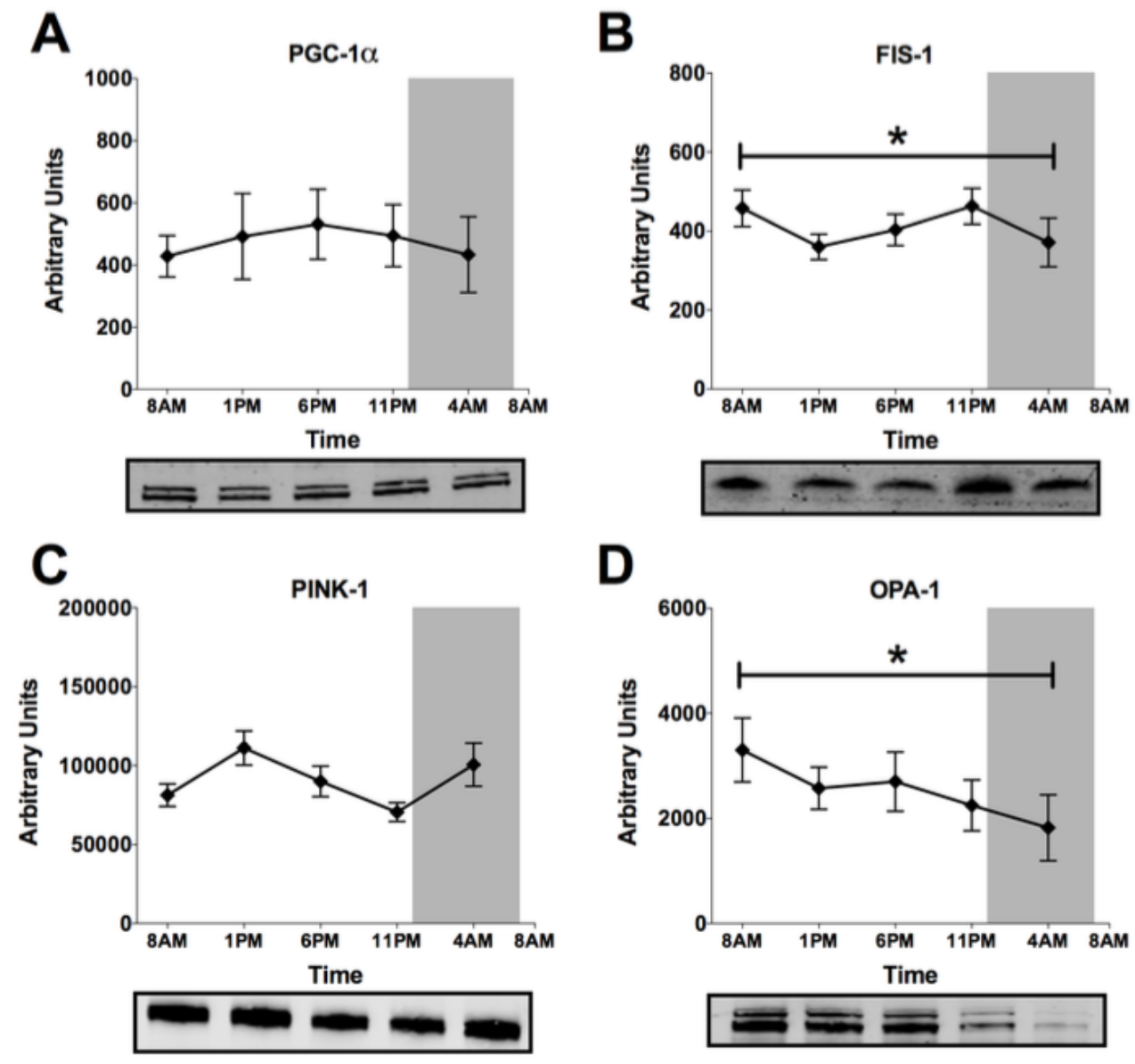

Figure 6: Mitochondrial dynamics rather than mitochondrial biogenesis displays a significant

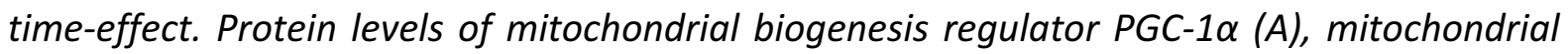
fission protein FIS-1 (B), mitophagy protein PINK-1 (C) and mitochondrial fusion protein OPA1 (D). Representative western blot images are displayed below their respective graphs.

It is well known that lowest levels of energy expenditure can be measured when subjects are asleep, denoted as sleeping metabolic rate. Even though the participants were awakened before measurements, resting energy expenditure was still lowest at 4AM. This clearly illustrates day-night rhythmicity in energy expenditure, independent of sleep. RER, carbohydrate oxidation and fat oxidation, displayed variation with feeding and fasting periods, with a significantly lower carbohydrate oxidation and RER, and a higher fat 

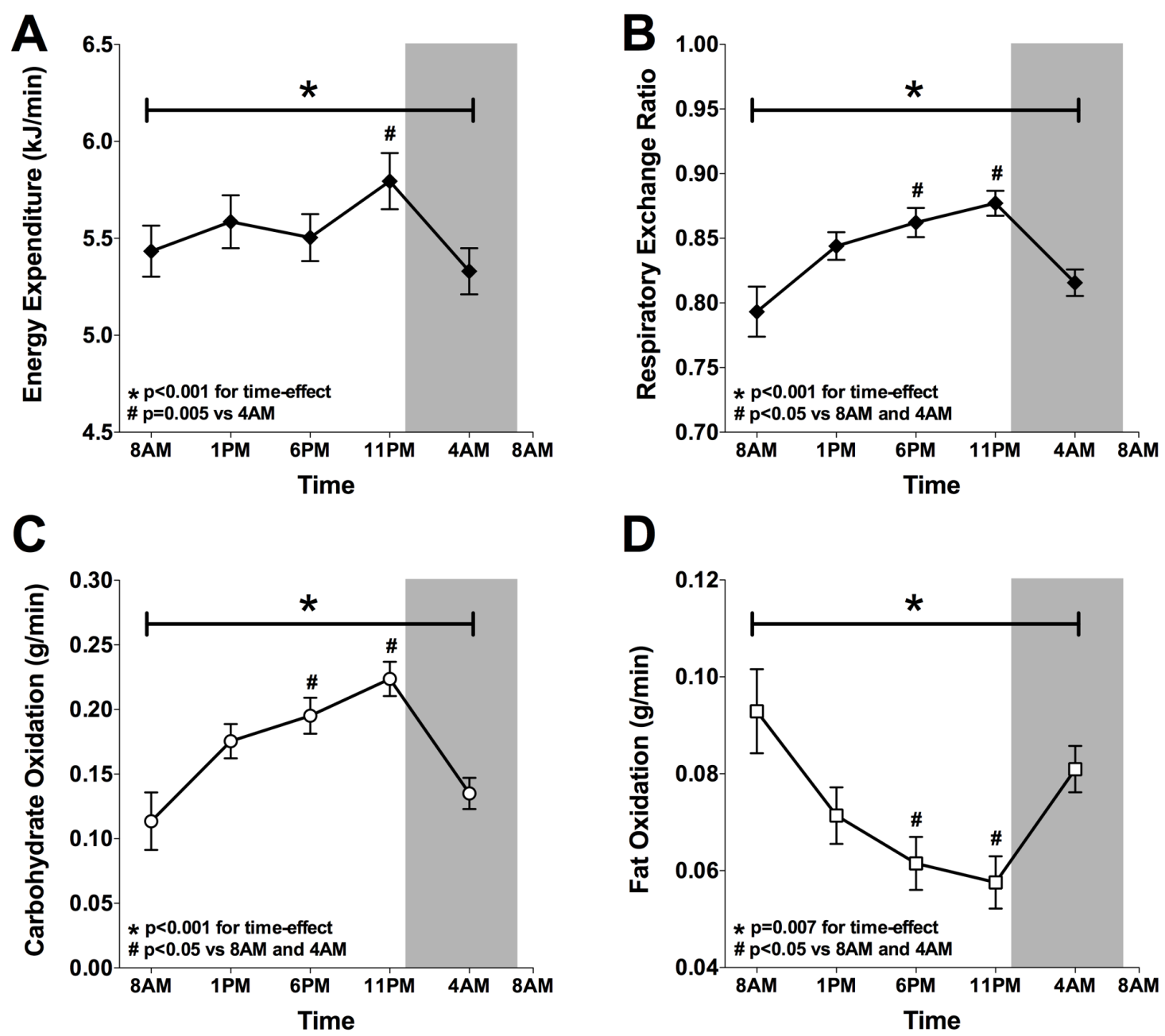

Figure 7: Whole-body resting energy expenditure peaks at the same time as skeletal muscle oxidative capacity whereas substrate oxidation exhibits a clear feeding and fasting pattern. Whole-body resting energy expenditure $(A)$, respiratory exchange ratio $(B)$, carbohydrate oxidation (C) and fat oxidation (D) during the second study-day, calculated from oxygen consumption and carbondioxide production measured by indirect calorimetry. Data presented as mean \pm SEM. ${ }^{*} p<0.01$ for the effect of time. $\# p<0.05$ for Bonferroni-adjusted post-hoc analysis.

oxidation in the fasted state (8AM and $4 A M$ ) and a higher carbohydrate oxidation and RER, and a lower fat oxidation in the fed state (1PM, 6PM and 11PM) $(p<0.001$ for the time-effect in RER and carbohydrate oxidation and $p=0.007$ for the time-effect in fat oxidation, figure 7B,C,D). Bonferroni-adjusted post-hoc analyses confirmed that RER, carbohydrate- and fat- 
A
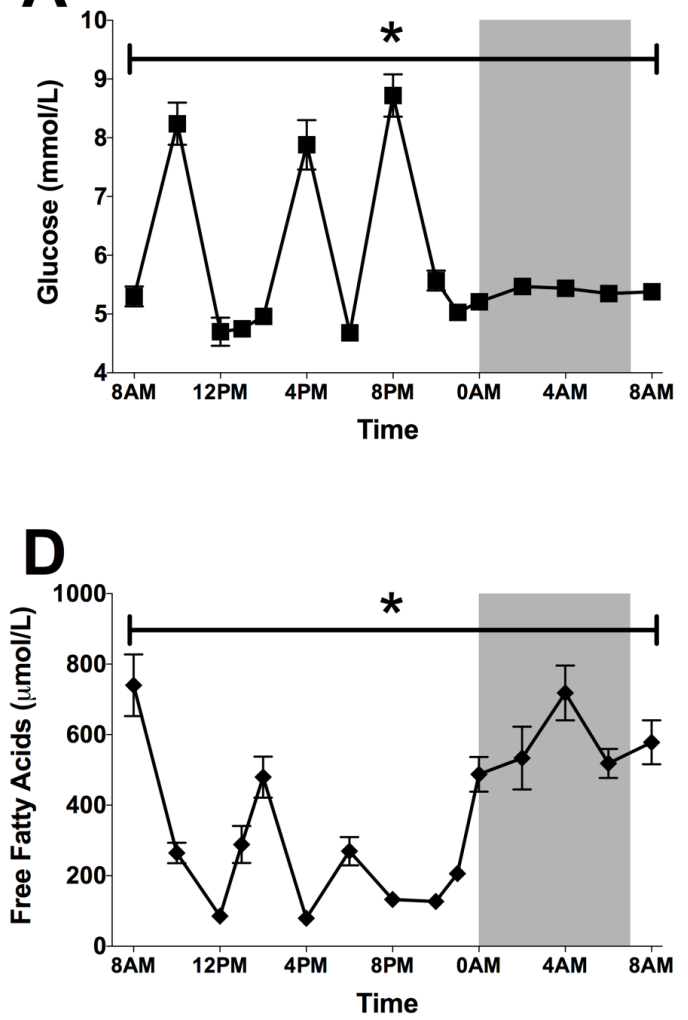

B

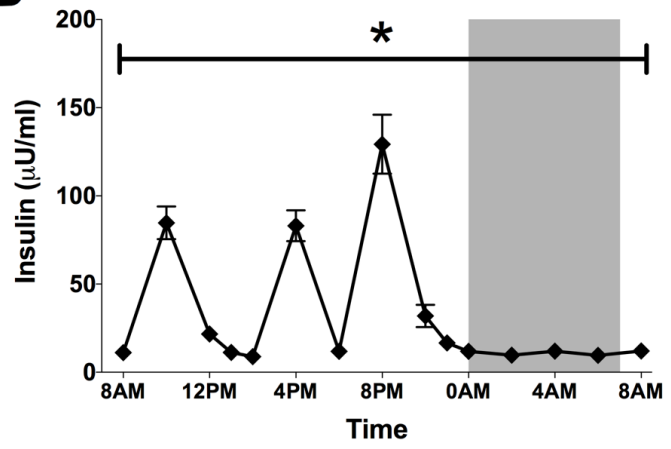

C

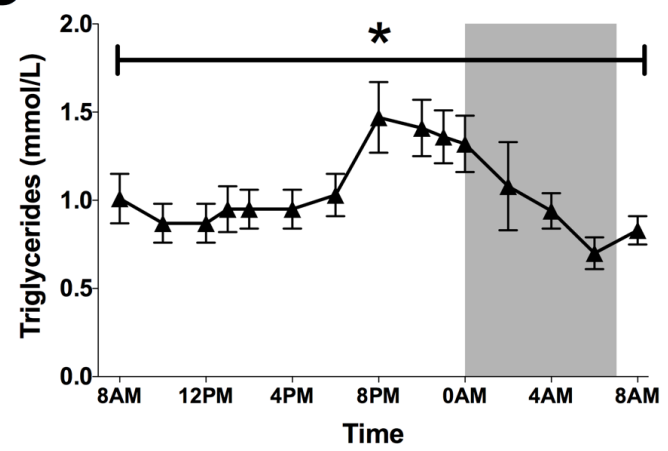

Figure 8: Plasma metabolites and insulin display marked variations over 24 hours, mostly associated with feeding-fasting. Plasma levels of glucose (A), insulin (B), free fatty acids (C) and triglycerides $(D)$ throughout the second-study day. Data depicted as mean \pm SEM. ${ }^{*} p<0.001$ for the effect of time.

oxidation were significantly different at 6 PM and 11PM, when compared with 8AM and 4AM ( $p<0.05$; figure 7A,B,C,D).

\section{Plasma metabolites peak according to meals}

To examine the diurnal pattern in circulating substrates, we took 15 blood samples throughout the second study-day to assess glucose, insulin, free fatty acids and triglycerides (figure 8A-D). As expected, plasma glucose and insulin displayed marked peaks after the meals, with an overall maximum after the dinner, being the most energy dense meal. Furthermore, plasma glucose increased slightly during the late evening and night, possibly reflecting an increase in endogenous glucose production together with decreased 
carbohydrate oxidation. Free fatty acids reflected the fasting-feeding pattern with increased free fatty acids before the meals and during the night when fat oxidation is highest and decreased free fatty acids after the meals. Plasma triglycerides displayed a rise throughout the waking day with a peak after dinner, being the meal with the highest energy density. During the night, plasma triglycerides decreased again.

\section{Circadian vs. day-night rhythmicity}

In this study, we aimed to examine changes in skeletal muscle mitochondrial function and whole-body metabolism throughout 24 hours with a standardized protocol designed to mimic our daily lifestyle. To this end, our protocol included a normal sleep-wake cycle, normal meals with a regular calorie distribution and (limited) physical activity. As a result, our protocol does not allow us to evaluate the influence of the endogenous circadian clock per se. However, to control the influence of meals and physical activity, we performed our measurements under controlled conditions with meals and light activity bouts at least 3-4 hours before the measurements. Such a protocol therefore reflects day-night, and not circadian rhythmicity, and allows extrapolation of the results to a normal daily lifestyle. The demonstration of a day-night rhythm in mitochondrial function in lean volunteers allows future studies to investigate if such rhythmicity is disturbed in volunteers with compromised metabolic health, such as T2DM. If rhythmicity of mitochondrial metabolism is indeed disturbed in subjects with compromised metabolic health, this may open therapeutic strategies to restore circadian rhythmicity possibly via REV-ERB- $\alpha$ agonists or nutritional compounds as resveratrol, as they have previously been proven to be successful in mouse studies $[42,43]$. Furthermore, it may open a new field of research directed towards the timing of interventions that boost mitochondrial function, and ultimately these studies may reveal how circadian disruption can lead to metabolic disturbances, as has been demonstrated in both human and animal studies $[6,7,44-46]$. 


\section{Conclusion}

In conclusion, we here demonstrate the presence of a profound day-night rhythm in human skeletal muscle mitochondrial oxidative capacity. Peak oxidative capacity and highest resting energy expenditure coincide at the end of the day, thereby partly matching the time phase of peak physical performance as described in literature [40, 41]. In addition, we found significant variations over time in proteins involved in mitochondrial dynamics, possibly linking the circadian clock with mitochondrial metabolism. Future investigations should increase the focus on the regulatory mechanisms underlying the current observations. Moreover, it should be examined if disturbances of the rhythm in human skeletal muscle oxidative capacity play a pivotal role in the development of the adverse metabolic consequences of circadian misalignment. 


\section{Supplemental figures}

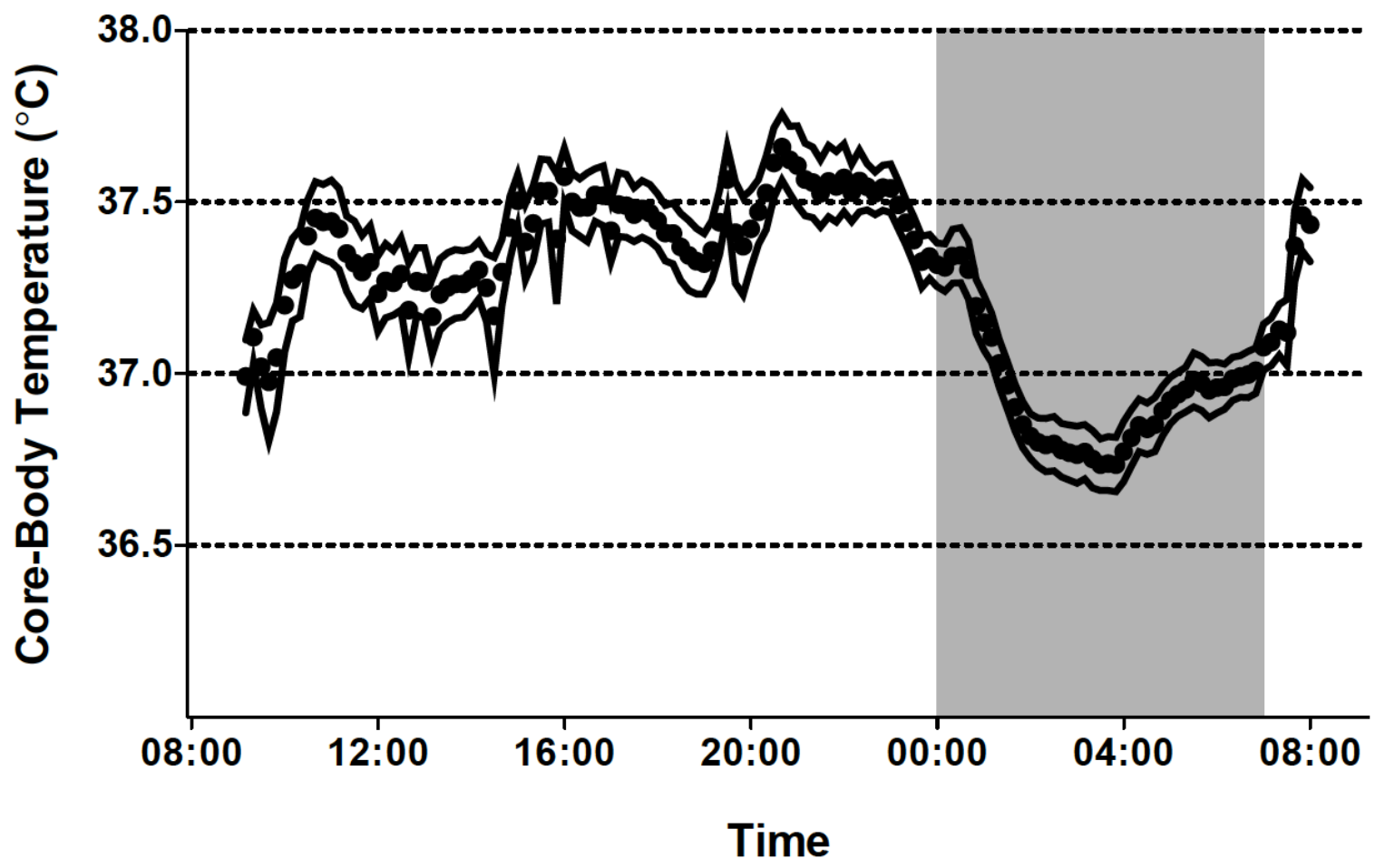

Supplemental figure 1: Core-body temperature displayed a characteristic day-night rhythm.

Core-body temperature measurements throughout the second study-day. Dots represent mean values of 10-minute averages, lines depict SEM. $p<.001$ for the effect of time. 


\section{References}

[1] Bailey SM, Udoh US, \& Young ME (2014) Circadian regulation of metabolism. J Endocrinol 222(2):R75-96.

[2] Gan Y, et al. (2015) Shift work and diabetes mellitus: a meta-analysis of observational studies. Occupational and environmental medicine 72(1):72-78.

[3] Buchvold HV, Pallesen S, Oyane NM, \& Bjorvatn B (2015) Associations between night work and $\mathrm{BMI}$, alcohol, smoking, caffeine and exercise--a cross-sectional study. BMC Public Health 15(1):1112.

[4] Karlsson B, Knutsson A, \& Lindahl B (2001) Is there an association between shift work and having a metabolic syndrome? Results from a population based study of 27,485 people. Occupational and environmental medicine 58(11):747-752.

[5] Ruger M \& Scheer FA (2009) Effects of circadian disruption on the cardiometabolic system. Rev Endocr Metab Disord 10(4):245-260.

[6] Scheer FA, Hilton MF, Mantzoros CS, \& Shea SA (2009) Adverse metabolic and cardiovascular consequences of circadian misalignment. Proceedings of the National Academy of Sciences of the United States of America 106(11):4453-4458.

[7] Morris CJ, et al. (2015) Endogenous circadian system and circadian misalignment impact glucose tolerance via separate mechanisms in humans. Proceedings of the National Academy of Sciences of the United States of America 112(17):E2225-2234.

[8] Leproult R, Holmback U, \& Van Cauter E (2014) Circadian misalignment augments markers of insulin resistance and inflammation, independently of sleep loss. Diabetes 63(6):1860-1869.

[9] Mohawk JA, Green CB, \& Takahashi JS (2012) Central and peripheral circadian clocks in mammals. Annu Rev Neurosci 35:445-462.

[10] Wolff G \& Esser KA (2012) Scheduled exercise phase shifts the circadian clock in skeletal muscle. Med Sci Sports Exerc 44(9):1663-1670.

[11] Stokkan KA, Yamazaki S, Tei H, Sakaki Y, \& Menaker M (2001) Entrainment of the circadian clock in the liver by feeding. Science 291(5503):490-493.

[12] Kumar Jha $P$, Challet $E$, \& Kalsbeek A (2015) Circadian rhythms in glucose and lipid metabolism in nocturnal and diurnal mammals. Molecular and cellular endocrinology 418 Pt 1:74-88.

[13] Peek CB, et al. (2013) Circadian clock NAD+ cycle drives mitochondrial oxidative metabolism in mice. Science 342(6158):1243417.

[14] Andrews JL, et al. (2010) CLOCK and BMAL1 regulate MyoD and are necessary for maintenance of skeletal muscle phenotype and function. Proceedings of the National Academy of Sciences of the United States of America 107(44):1909019095.

[15] Jacobi D, et al. (2015) Hepatic Bmal1 Regulates Rhythmic Mitochondrial Dynamics and Promotes Metabolic Fitness. Cell metabolism 22(4):709-720.

[16] Neufeld-Cohen A, et al. (2016) Circadian control of oscillations in mitochondrial ratelimiting enzymes and nutrient utilization by PERIOD proteins. Proceedings of theNational Academy of Sciences of the United States of America.

[17] Phielix E, et al. (2008) Lower intrinsic ADP-stimulated mitochondrial respiration underlies in vivo mitochondrial dysfunction in muscle of male type 2 diabetic patients. Diabetes 57(11):2943-2949. 
[18] Bakkman L, et al. (2010) Reduced respiratory capacity in muscle mitochondria of obese subjects. Obes Facts 3(6):371-375.

[19] Scheer FAS, S.A. (2011) Fundamentals of the Circadian System. in SRS Basics of Sleep Guide (Sleep Research Society), pp 199 - 210.

[20] Schoffelen PF, Westerterp KR, Saris WH, \& Ten Hoor F (1997) A dual-respiration chamber system with automated calibration. J Appl Physiol (1985) 83(6):2064-2072.

[21] Harris JA \& Benedict FG (1918) A Biometric Study of Human Basal Metabolism. Proceedings of the National Academy of Sciences of the United States of America 4(12):370-373.

[22] Bergstrom J, Hermansen L, Hultman E, \& Saltin B (1967) Diet, muscle glycogen and physical performance. Acta physiologica Scandinavica 71(2):140-150.

[23] Hoeks J, et al. (2010) Prolonged fasting identifies skeletal muscle mitochondrial dysfunction as consequence rather than cause of human insulin resistance. Diabetes 59(9):2117-2125.

[24] Weir JB (1949) New methods for calculating metabolic rate with special reference to protein metabolism. J Physiol 109(1-2):1-9.

[25] Peronnet F \& Massicotte D (1991) Table of nonprotein respiratory quotient: an update. Can J Sport Sci 16(1):23-29.

[26] Tripathi S, et al. (2015) Meta- and Orthogonal Integration of Influenza "OMICs" Data Defines a Role for UBR4 in Virus Budding. Cell Host Microbe 18(6):723-735.

[27] Hellemans J, Mortier G, De Paepe A, Speleman F, \& Vandesompele J (2007) qBase relative quantification framework and software for management and automated analysis of real-time quantitative PCR data. Genome Biol 8(2):R19.

[28] Hughes ME, Hogenesch JB, \& Kornacker K (2010) JTK_CYCLE: an efficient nonparametric algorithm for detecting rhythmic components in genome-scale data sets. Journal of biological rhythms 25(5):372-380.

[29] Krauchi K (2007) The human sleep-wake cycle reconsidered from a thermoregulatory point of view. Physiol Behav 90(2-3):236-245.

[30] Bonaconsa M, et al. (2014) Differential modulation of clock gene expression in the suprachiasmatic nucleus, liver and heart of aged mice. Exp Gerontol 55:70-79.

[31] Archer SN, Viola AU, Kyriakopoulou V, von Schantz M, \& Dijk DJ (2008) Interindividual differences in habitual sleep timing and entrained phase of endogenous circadian rhythms of BMAL1, PER2 and PER3 mRNA in human leukocytes. Sleep 31(5):608-617.

[32] Akashi M, et al. (2010) Noninvasive method for assessing the human circadian clock using hair follicle cells. Proceedings of the National Academy of Sciences of the United States of America 107(35):15643-15648.

[33] Otway DT, et al. (2011) Rhythmic diurnal gene expression in human adipose tissuefrom individuals who are lean, overweight, and type 2 diabetic. Diabetes 60(5):1577-1581.

[34] Perrin L, et al. (2015) Human skeletal myotubes display a cell-autonomous circadian clock implicated in basal myokine secretion. Mol Metab 4(11):834-845.

[35] Dyar KA, et al. (2014) Muscle insulin sensitivity and glucose metabolism are controlled by the intrinsic muscle clock. Mol Metab 3(1):29-41. 
[36] Phielix E, Meex R, Moonen-Kornips E, Hesselink MK, \& Schrauwen P (2010) Exercise training increases mitochondrial content and ex vivo mitochondrial function similarly in patients with type 2 diabetes and in control individuals. Diabetologia 53(8):1714-1721.

[37] Kudo T, et al. (2004) Night-time restricted feeding normalises clock genes and Pai-1 gene expression in the $\mathrm{db} / \mathrm{db}$ mouse liver. Diabetologia 47(8):1425-1436.

[38] Kohsaka A, et al. (2007) High-fat diet disrupts behavioral and molecular circadian rhythms in mice. Cell metabolism 6(5):414-421.

[39] Dahlmans D, Houzelle A, Schrauwen P, \& Hoeks J (2016) Mitochondrial dynamics, quality control and miRNA regulation in skeletal muscle: implications for obesity and related metabolic disease. Clin Sci (Lond) 130(11):843-852.

[40] Conroy RT \& O'Brien M (1974) Proceedings: Diurnal variation in athletic performance. J Physiol 236(1):51P.

[41] Facer-Childs E \& Brandstaetter R (2015) The impact of circadian phenotype and time since awakening on diurnal performance in athletes. Curr Biol 25(4):518-522.

[42] Woldt E, et al. (2013) Rev-erb-alpha modulates skeletal muscle oxidative capacity by regulating mitochondrial biogenesis and autophagy. Nature medicine 19(8):10391046.

[43] Liu J, et al. (2016) CLOCK and BMAL1 Regulate Muscle Insulin Sensitivity via SIRT1 in Male Mice. Endocrinology 157(6):2259-2269.

[44] Arble DM, Bass J, Laposky AD, Vitaterna MH, \& Turek FW (2009) Circadian timing of food intake contributes to weight gain. Obesity 17(11):2100-2102.

[45] Bandin C, et al. (2015) Meal timing affects glucose tolerance, substrate oxidation and circadian-related variables: A randomized, crossover trial. Int J Obes (Lond) 39(5):828-833.

[46] Salgado-Delgado RC, et al. (2013) Shift work or food intake during the rest phase promotes metabolic disruption and desynchrony of liver genes in male rats. PloS one 8(4):e60052. 


\section{CHAPTER 6}

\section{Circadian Misalignment Induces Insulin Resistance and affects the skeletal muscle Molecular Clock in Humans}

\section{Authors}

Jan Hansen", Jakob Wefers", Dirk van Moorsel" Helene Duez, Bart Staels, Frank AJL. Scheer, Esther Phielix, Matthijs KC. Hesselink, Patrick Schrauwen

$=$ These authors contributed equally. 


\section{Abstract}

Background: Circadian misalignment, such as in shift work, has been associated with obesity and insulin resistance. Animal studies have shown that the molecular circadian clock in skeletal muscle is necessary for insulin sensitivity and glucose tolerance. However, direct effects of circadian misalignment on insulin sensitivity and the circadian clock have never been investigated in humans. We hypothesized that circadian misalignment leads to a decrease in insulin sensitivity and to a disturbed molecular clock in skeletal muscle.

Methods: Fourteen healthy young lean men (age $22.4 \pm 2.8$ years; BMI $22.3 \pm 2.1$ $\mathrm{kg} / \mathrm{m}^{2}$ [mean $\pm \mathrm{SD}$ ]) participated in a randomized cross-over study to determine the influence of controlled circadian misalignment on insulin sensitivity, assessed by hyperinsulinemic two-step euglycemic clamping. Participants were studied after a 3day control period and after a 3.5-day misalignment period performed by a $12-\mathrm{h}$ rapid shift in a respiration chamber, isolated from external time cues. Glucose, FFA and triglyceride levels were determined in plasma samples after an overnight fast (either at 7 AM or 7 PM). We also performed muscle biopsies in the morning and evening of the last test of each study condition (7 AM and 7 PM).

Results: Circadian misalignment resulted in a significant decrease in peripheral insulin sensitivity, which was almost completely due to non-oxidative glucose disposal in skeletal muscle (Rate of disappearance: $35.2 \pm 2.4$ vs. $30.3 \pm 1.3$ $\mathrm{mg} / \mathrm{kg} / \mathrm{min}$; control vs. misalignment; $\mathrm{p}=0.029$ ). Fasting glucose and FFA levels were higher in circadian misalignment (Glucose: $5.0 \pm 0.1$ vs. $5.2 \pm 0.1 \mathrm{mmol} / \mathrm{l}$; control vs. misalignment; $p=0.015$; FFA: $389 \pm 47$ vs. $525 \pm 48 \mu \mathrm{mol} / \mathrm{l}$; control vs. misalignment; $p=0.006)$. Sleeping (4.96 \pm 0.12 vs. $5.16 \pm 0.15 \mathrm{~kJ} / \mathrm{min}$; control vs. misalignment, $p=$ $0.014)$ and resting metabolic rate $(5.09 \pm 0.17 \mathrm{vs} .5 .35 \pm 0.16 \mathrm{~kJ} / \mathrm{min}$; control vs. misalignment; $p=0.14$ ) were higher during misalignment.

Intriguingly, skeletal muscle gene expression of the circadian clock genes BMAL1, PER2, CRY2, REV-ERB- $\alpha$ did not align to the new behavioral rhythm, illustrating misalignment of the core molecular clock in human skeletal muscle.

Conclusions: Controlled circadian misalignment leads to decreased peripheral insulin sensitivity and disturbed glucose, FFA levels and energy metabolism. Moreover, in circadian misalignment the skeletal muscle circadian clock was misaligned to the behavioral routine. Our findings show that circadian misalignment leads to metabolic disturbances in healthy subjects, which supports a causal role in the development of type 2 diabetes mellitus. 


\section{Introduction}

The dramatic increase in the prevalence of obesity and type 2 diabetes mellitus (T2DM) is an important global health issue, as it comes with high morbidity and mortality and poses a major burden on health care costs. Obesity and type 2 diabetes mellitus are both strongly associated with a westernized lifestyle of low physical activity levels and high food intake. However, recently it has been recognized that also our 24-hour culture, characterized by working and eating late, reduced sleep (quality) and excessive light exposure, should be considered as a lifestyle factor that may negatively impact metabolic health. Indeed, observational studies show that circadian misalignment, such as in shift work, is associated with adverse metabolic consequences and increased risk to develop T2DM [1-3]. Moreover, laboratory studies with controlled circadian misalignment have demonstrated detrimental effects on (postprandial) glucose- and insulin levels, which may indicate decreased insulin sensitivity [4-6].

Circadian rhythmicity of metabolism is regulated by the core circadian clock, which consists of a transcriptional-translational feedback loop, in which the transcriptional activators BMAL1 and CLOCK induce expression of their own repressors CRY and PER, generating 24-hour oscillations which affect up to $\sim 20 \%$ of the genome transcripts. Importantly, circadian oscillators are present throughout every body tissue, including skeletal muscle $[7,8]$, which is a major organ involved in the regulation of glucose homeostasis. The circadian clock is increasingly recognized as key regulator of many metabolic processes, and serves to anticipate metabolic challenges and demands across a normal day [9]. Importantly, systemic or localized disruption of circadian rhythm in animal models has been associated with impaired glucose metabolism and the development of mitochondrial dysfunction. Ablation of BMAL1 in mice resulted in decreased fatty acid oxidation as well as oxygen consumption rates of mitochondria $[10,11]$. Moreover, mice with liver or skeletal muscle specific ablation of BMAL1 developed insulin resistance $[12,13]$.

So far, evidence that disturbing the day-night rhythm can also affect skeletal muscle metabolism in humans is limited. We have previously shown that also in humans, skeletal muscle is characterized by a day-night rhythm in among others molecular clock gene expression and mitochondrial respiratory capacity. It is known that mitochondrial function is disturbed in the insulin resistant state $[14,15]$, but so far it is unknown if disturbing day-night rhythm in humans also leads to disturbed skeletal muscle metabolism and/or insulin resistance. 
We here investigated if circadian misalignment would lead to disturbances in muscle metabolism and insulin resistance in human volunteers. To this end, we performed a detailed human translational intervention study in which insulin sensitivity assessed by hyperinsulinemic euglycemic clamp - and molecular analysis of muscle biopsies was performed after a 3-day control condition and after a 3.5-day period in which day-night rhythm was shifted by 12 hours.

\section{Material and methods}

\section{Participants}

Fourteen healthy lean young men (Age: $22.4 \pm 2.8$ years; BMI: $22.3 \pm 2.1 \mathrm{~kg} / \mathrm{m}^{2}$; Mean \pm SD) participated in the study. Participants were non-smokers, had no active diseases, used no medication and did not engage in exercise for more than 3 hours per week, verified by questionnaires. In addition, participants reported regular bedtimes (11 PM \pm 2 hours), regular sleep duration of 7-9 hours, did not perform shift-work or traveled across more than one time zone in the last three months and were not morning larks or night owls, assessed with a morningness-eveningness questionnaire (MEQ-SA: $54 \pm 7$, Mean \pm SD). The study was conducted in accordance with the principles of the declaration of Helsinki, approved by the Ethics Committee of the Maastricht University Medical Center and monitored by the Clinical Trial Center Maastricht. All participants provided written informed consent. The included measurements were performed between February 2016 and February 2017. The study was registered at clinicaltrials.gov with identifier NCT02580513.

\section{Pre-study conditions}

Seven days prior to the study periods, participants were instructed to maintain a standardized normal lifestyle, including (trying to) sleep from 11 PM until 7 AM and consuming meals at regular times, consistent with the times during the study (breakfast, lunch, snack and dinner at 8 AM, 12:30 PM, 3 PM and 8 PM). In additions, participants were asked to abstain from consumption of caffeine and alcohol. Three days before the study periods, participants were instructed not to perform physical exercise. Two days before the study periods, participants were provided with meals to ensure standardized distribution of calories and macronutrients (see below). Compliance to the prescribed lifestyle was monitored with food-and sleep diaries and by a light-detecting wrist accelerometer (Actiwatch Spectrum, PhilipsRespironics, Murrysville PA, USA). 


\section{Study conditions}

In the study, each participant underwent one control period and one period in circadian misalignment, in a randomized, crossover fashion, with a wash-out of 3 to 9 weeks. Prior to the first study period, the participants were not aware of the order of the study periods. During their stay, the participants resided in a private respiration chamber; a small room with a bed, toilet, sink, desk, chair, TV and computer. In the respiration chamber; environmental conditions were tightly controlled, including dim-light (4 lux in the horizontal angle of gaze) during wake phases and darkness during sleep opportunities, no devices that can display time (e.g. watch, mobile phone, laptop, tablet), no internet and no live television. The screen emittance of the TV and computer in the respiration chamber were adapted to not exceed the permitted light intensity of 4 lux and could be used to work, listen to music or watch movies. During their stay, participants were isolated from external time cues and could only be in contact with the researchers. For specific study procedures (placement of intravenous canula, indirect calorimetry, skeletal muscle biopsy and the hyperinsulinemic euglycemic clamp), participants were transported to an adjacent clinical room in a wheelchair, during which they wore a blindfold and earplugs to prevent excess light and possible audiovisual signals that might indicate whether it was day or night. The clinical room had similar dim-light conditions. During procedures for which light was required (placement of intravenous canula, muscle biopsy), participants wore welding goggles shade 5 (Uvex Ultravision 9301245, Uvex, Fürth, Germany) to limit light exposure.

\section{Study design}

The study design is graphically depicted in figure 1 . For the control period, participants were admitted to the research unit at 7:45 PM on day 1 and stayed until 5PM on day 4. Sleep opportunities were from 11 PM until 7 AM, during which participants were required to switch off the lights and try to sleep. Participants were not allowed to sleep during wake periods. During the test days (except for the last test-day), participants received breakfast, lunch, a snack and dinner (see below) at fixed times (8 AM, 12:30 PM, 15 PM and 8 PM, respectively). One hour after each meal, participants were instructed to perform a 30-minute, light physical activity, consisting of $3 \times 5$ minutes of step exercises and $3 \times 5$ minutes of standing. In addition, participants were instructed to follow a standardized lifestyle with fixed activities (making the bed, doing the dishes, 15-minutes standing, sitting at desk or free time) to prevent differences between the 2 study conditions. Sleeping metabolic rate was 


\section{Control condition}

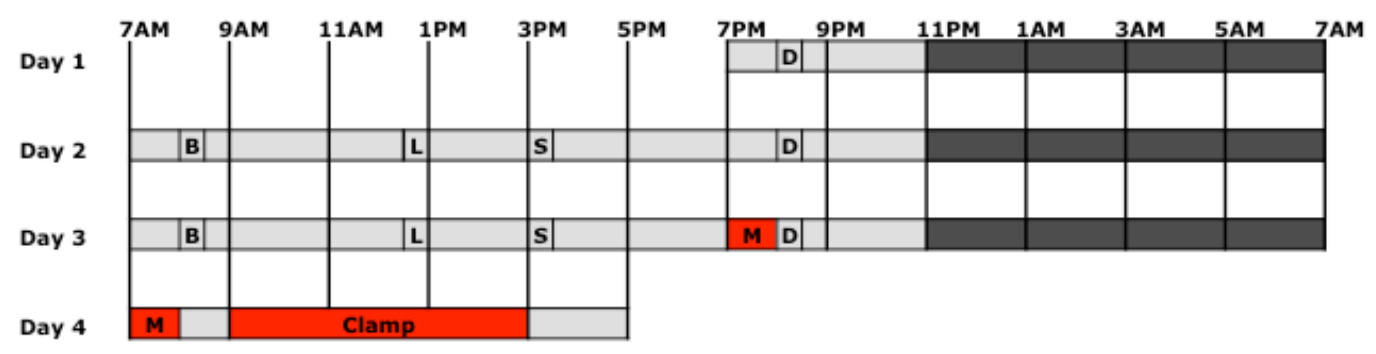

\section{Misalignment condition}

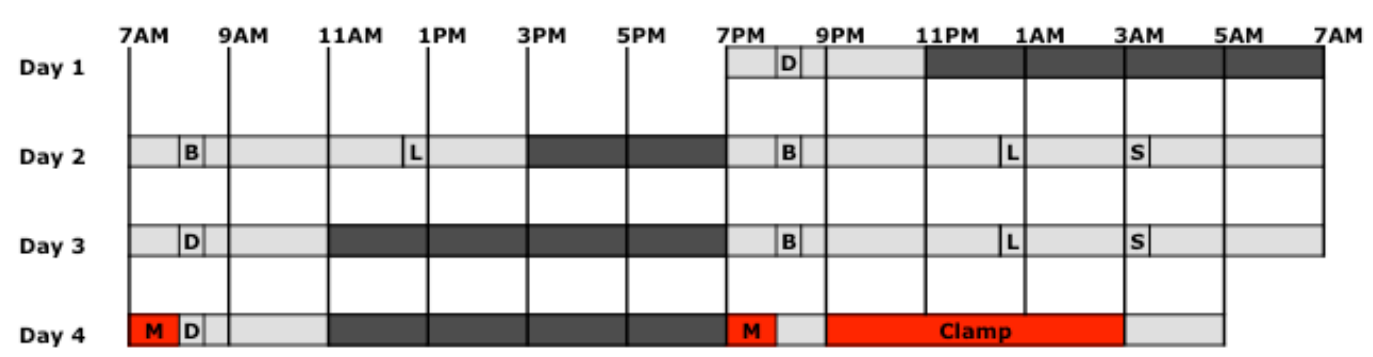

Figure 1: Study design

Participants underwent a control and a misalignment condition in a randomized cross-over design. $B$, breakfast; $L$, lunch; $S$, snack; $D$, dinner; $M$, measurement of blood substrates, energy metabolism and muscle biopsy; Clamp, hyperinsulinemic euglycemic clamp. Gray bars indicate waking hours with 4 lux ambient light, black bars indicate sleep opportunity with lights out.

measured by whole-room indirect calorimetry (Omnical, Maastricht Instruments, Maastricht, The Netherlands) during the first night, which was used for estimation of caloric requirements.

After waking up on the third study day, participants ingested a thermometer capsule (Equivital, Philips-Respironics, Murrysville PA, USA) to measure core-body temperature (CBT) for 24 hours. Due to early excretion of the capsule or technical failure complete CBT measurements were obtained in 8 participants. At 7 PM, participants underwent a blood draw, directly followed by a 30-minute resting indirect calorimetry measurement and a skeletal muscle biopsy (see below). During the sleep opportunity between day 3 and 4 , we measured sleeping metabolic rate in 
the respiration chamber by whole-room indirect calorimetry (Omnical, Maastricht Instruments, Maastricht, The Netherlands).

On the fourth study day, participants were awakened at 6:45 AM. Starting at 7 AM, an intravenous cannula was placed in the antecubital vein, followed by a blood draw, indirect calorimetry and skeletal muscle biopsy, similar to the previous day. After the biopsy, a two-step hyperinsulinemic euglycemic clamp was performed to assess peripheral and hepatic insulin sensitivity (see below). During this test, participants stayed fasted. Directly after this test (which takes approximately 8 hours), participants were provided with a meal and were allowed to go home as soon as plasma glucose was stabilized.

For the misaligned period, participants were also admitted at 7:45 PM on day 1 and had a normal sleeping opportunity from 11 PM until 7 AM. On day 2, we imposed circadian misalignment by shifting the behavioral cycle by 12 hours. This was realized by instructing participants to switch off the lights, lie in bed and try to sleep from 3 PM until 7 PM. At 7 PM, participants were awakened and started a new behavioral morning. From this point, study procedures are equal to the control period, but shifted by 12 hours (misalignment 7 PM day 2 is equal to control 7 AM day 2, see figure 1). Consequently, breakfast, lunch, snack and dinner are served at 8 PM, 12:30 $A M, 3 A M$ and 8 AM and consecutive sleep opportunities were scheduled from 11 AM until 7 PM. The blood draw, indirect calorimetry and skeletal muscle biopsies were performed on day 4 at 7 AM (behavioral evening) and at 7 PM (behavioral morning). The hyperinsulinemic euglycemic clamp started after the 7 PM biopsy.

\section{Study meals}

Two days before and during the study, participants were provided with standardized meals according to Dutch and US dietary guidelines. Caloric requirements for consumption at home and the first evening of the study period were calculated by multiplying the resting metabolic rate, estimated using the Harris-Benedict formula, with an activity factor of 1.5. In addition, as it is difficult to predict the exact energy expenditure of the participants, we provided additional snacks to reach an energy intake equal to the resting metabolic rate multiplied by a factor of 1.7. Participants were allowed to eat these additional snacks directly after their meals, only if they were still hungry. Caloric requirements for consecutive study days were estimated by 
multiplying the recorded sleeping metabolic rate (see below) with an activity factor of 1.5 .

Caloric intake was divided over 3 daily meals and a snack. Breakfast accounted for $\sim 20$ energy\%, lunch for $\sim 25$ energy\%, a snack for $\sim 10$ energy\% and dinner for $\sim 45$ energy\%. Daily macronutrient distribution was $\sim 52$ energy\% as carbohydrates, $\sim 31$ energy\% as fat ( $\sim 9 \%$ saturated) and $\sim 14$ energy\% as protein. Breakfast and lunch were bread meals, while dinner was a warm meal, resulting in a relatively higher fat content for dinner, as is common in The Netherlands. Besides the meals, no other snacks or drinks than water were provided.

\section{Indirect calorimetry}

To determine whole-body energy expenditure, respiratory exchange ratio (RER), glucose- and fat-oxidation, oxygen consumption and carbon dioxide production were measured with an automated respiratory gas analyzer using a ventilated hood system (Omnical; Maastricht Instruments, Maastricht, the Netherlands). Calculations of energy expenditure and substrate oxidation were made with the assumption of a negligible protein oxidation $[16,17]$. For sleeping metabolic rate, we used the lowest 3-hour mean energy expenditure of the sleep period, measured by whole-room calorimetry (Omnical, Maastricht Instruments, Maastricht, The Netherlands), extrapolated to 24-hour energy expenditure [18].

\section{Skeletal muscle biopsies and respirometry}

During each study period, two skeletal muscle biopsies were obtained according to the Bergström method [19] from the $m$. vastus lateralis under local anesthesia (1\% lidocaine, without epinephrine). Biopsies were taken moving from distal to proximal, alternating between the left or right leg. The leg of the first biopsy was randomized and subsequent biopsies were taken from the other leg, moving from distal to proximal. For the 7AM and 7PM biopsies, part of the biopsy was immediately placed in ice-cold preservation medium (BIOPS, OROBOROS Instruments, Innsbruck, Austria) and used for measurement of mitochondrial oxidative capacity. For this analysis, we permeabilized intact muscle fibers and measured their oxygen consumption upon several substrates using high-resolution respirometry (Oxygraph, OROBOROS Instruments, Innsbruck, Austria). Substrates used to consecutively supplement muscle fibers during this assay were malate, octanoylcarnitine, adenosine diphosphate (ADP), glutamate, succinate and carbonylcyanide $p$ - 
trifluoromethoxyphenylhydrazone (FCCP) (trace 1) or malate, pyruvate, ADP, glutamate, succinate and FCCP (trace 2), as described previously [20]. The remaining part of the muscle biopsy was immediately frozen in melting isopentane and stored in $-80^{\circ} \mathrm{C}$ until further analysis.

Mitochondrial electron transport chain complexes were quantified by western blot analysis as previously described [8]. Human muscle tissue was lysed in RIPA buffer. Protein concentration was determined using the Bio-Rad RC/DC kit (Bio-Rad Laboratories, Veenendaal, The Netherlands). Equal amounts of protein were loaded on 12\% TGX gels (Bio-Rad Laboratories). Proteins were transferred to nitrocellulose with the Trans-Blot Turbo transfer system (Bio-Rad Laboratories). Primary antibodies: a cocktail of mouse monoclonal antibodies directed against human OXPHOS (dilution 1:10,000; ab110411, Abcam, Cambridge, UK). The specific proteins were detected using secondary antibodies conjugated with IRDye800, and were quantified with the CLx Odyssey Near Infrared Imager (Li-COR, Westburg, Leusden, The Netherlands). The individual total intensity of Complex I, II, III, IV and V was quantified and is expressed in relative units (RU).

\section{Gene transcript quantification}

RNA was isolated from $50 \mathrm{mg}$ of muscle material by TRIzol lysis (Qiagen, Hilden, Germany). RNA was further purified by the RNeasy kit from Qiagen (Hilden, Germany). RNA yield was measured using a NanoDrop spectrophotometer (Thermo Fisher Scientific, Waltham, USA). The high-capacity RNA-to-cDNA kit from Applied Biosystems (Foster City, USA) was used for transcribing 0.5 mg RNA to cDNA. Transcript abundance was determined by RT-QPCR using a CFX348 Touch $^{\text {TM }}$ RealTime PCR detection system (Bio-Rad; Hercules, CA). To minimize the variability in reference gene normalization, we used the geometric mean of three reference genes (RPL26, GUSB and CYPB), which were previously demonstrated to be expressed robustly independent of time $[8,21]$ and metabolic phenotype [21]. This geometric mean was used as the internal reference for comparative gene expression analysis in the remainder of the study.

\section{Hyperinsulinemic euglycemic clamp}

To determine peripheral and hepatic insulin sensitivity, we performed a two-step hyperinsulinemic euglycemic clamp with infusion of D-[6,6- $\left.{ }^{2} \mathrm{H}_{2}\right]$ glucose tracer [22]. First, a primed continuous infusion of D-[6,6- $\left.{ }^{2} \mathrm{H}_{2}\right]$ glucose was given for two hours to 
determine baseline endogenous glucose production (EGP), glucose appearance (Ra) and glucose disposal (Rd) [23]. Next, insulin was infused at a primed continuous low rate $\left(10 \mathrm{mU} / \mathrm{m}^{2} / \mathrm{min}\right)$ for 3 hours, to assess hepatic insulin sensitivity (suppression of EGP by insulin). Finally, insulin was infused at a primed high rate for 2 hours (40 $\mathrm{mU} / \mathrm{m}^{2} / \mathrm{min}$ ) to determine peripheral insulin sensitivity (glucose Rd). During insulin infusion, blood was frequently sampled and glucose levels directly analyzed in arterialized blood. Glucose was co-infused at an accordingly variable rate to maintain euglycemia $(5.0 \mathrm{mmol} / \mathrm{L})$. During the last 30 minutes of each step, substrate oxidation was measured by indirect calorimetry and blood was sampled every 10 minutes. Steele's single pool non-steady state equations were used to calculate Ra and $\mathrm{Rd}$ of glucose [24].We assumed a distribution volume of $0.160 \mathrm{~L} / \mathrm{kg}$ for glucose.

\section{Statistics}

Data are presented as mean \pm SEM (standard error of the mean) unless indicated otherwise. Statistical analyses were performed with the use of IBM Statistical Package for Social Sciences for MAC, version 23 (SPSS, Inc.). The effect of circadian misalignment on outcome variables was assessed by paired samples T-test. Statistical significance was defined as a p-value $<0.05$.

\section{Results}

\section{2-h rapid shift in behavior induces circadian misalignment}

In the current study, we used a rapid shift protocol to induce circadian misalignment. To test if the volunteers were indeed in circadian misalignment, we recorded core body temperature (CBT) using an ingested thermopill allowing the measurement of $24 \mathrm{~h}$ core body temperature. As can be seen in figure 1 , core body temperature follows a pronounced circadian rhythm, with high body temperature during waking hours, a decrease in the late evening and remaining low values during sleep (figure 2 A). Consistent with circadian misalignment, core body temperature remained high during the day even though subjects were sleeping during day time and decreased in the late evening when subjects were awake, indicating that the central circadian clock indeed was misaligned to the behavioral shift and maintained the circadian phase of the control condition (figure 2 B). Therefore, the rapid shift protocol was successful in inducing circadian misalignment. 
A

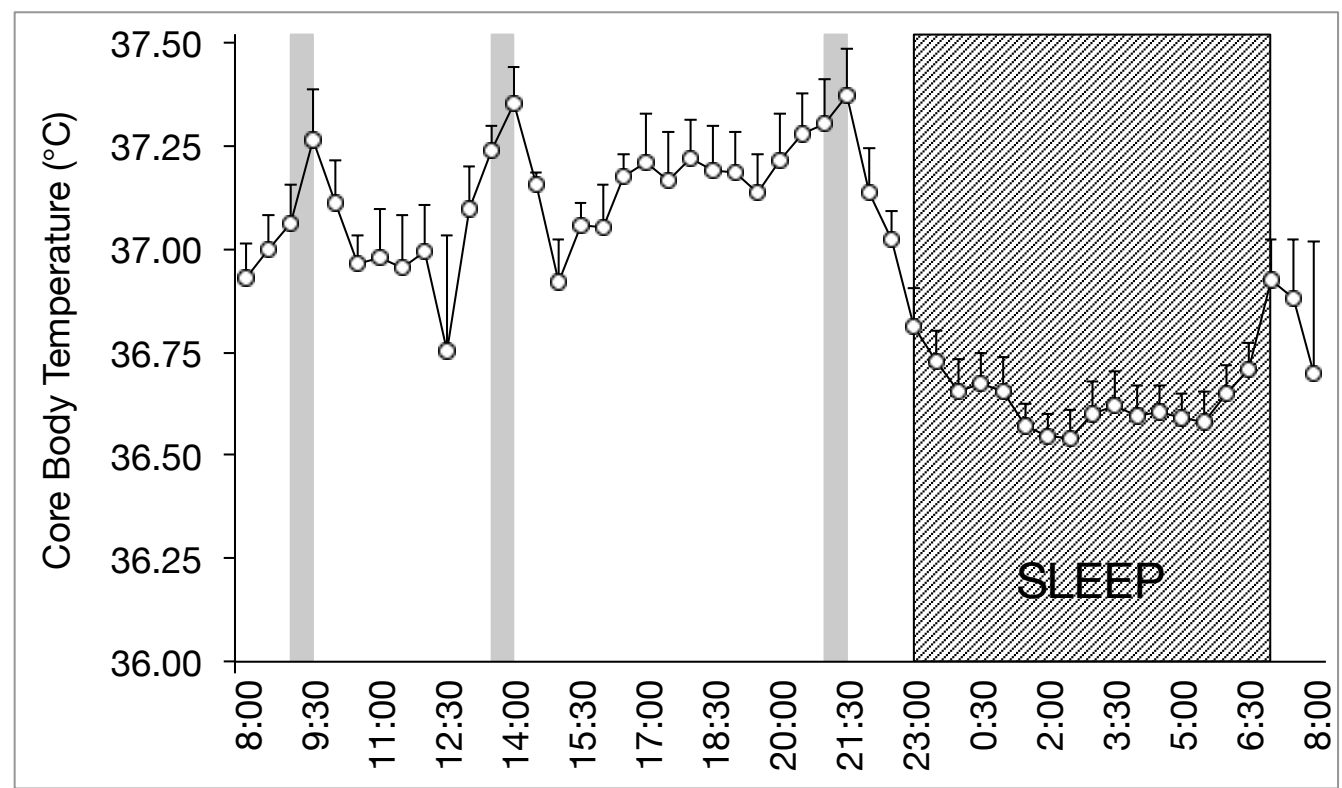

B

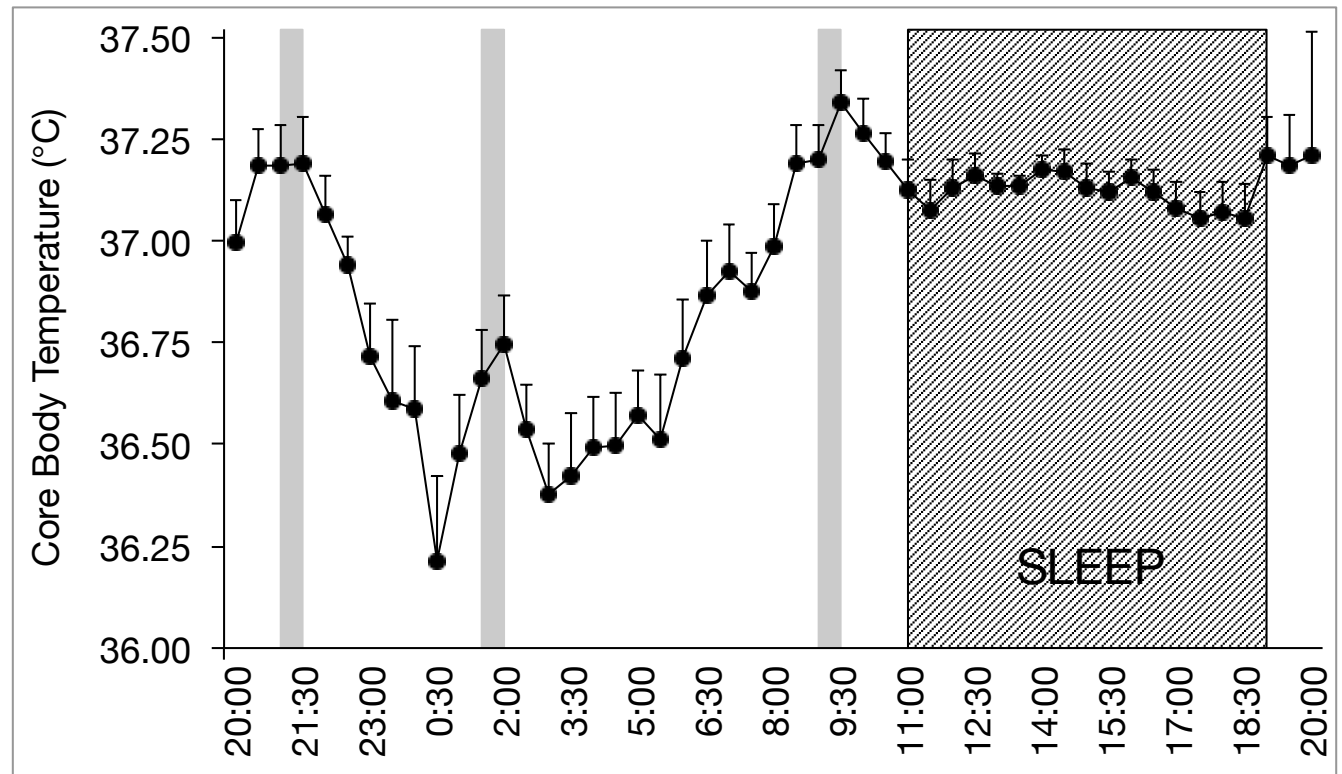

Figure 2: Core Body Temperature (CBT) in the control and misalignment condition Core body temperature $\left({ }^{\circ} \mathrm{C}\right)$ recordings were obtained every 15 seconds and averaged per subject into 30 min intervals. Depicted are control condition (A) and misalignment condition (B). Sleeping periods are indicated by wide grey bars. The small grey bars indicate 30 min bouts of physical activity performed one hour after each meal. Data is expressed as mean \pm SEM. ${ }^{*} p<0.05$. 


\section{Sleeping metabolic rate is higher during circadian misalignment}

To examine if circadian misalignment of body temperature was also reflected in alterations in energy expenditure, we determined whole-body sleeping metabolic rate using whole-body respiratory rooms. Sleeping metabolic rate was defined as the lowest energy expenditure during sleep for three consecutive hours. Consistent with higher body temperature during the sleeping phase, SMR was elevated upon circadian misalignment compared to control condition (4.96 \pm 0.12 vs. $5.16 \pm 0.15$ $\mathrm{kJ} / \mathrm{min}$, control vs. misalignment $\mathrm{p}=0.014$, figure 3 ). Substrate oxidation during sleep was not affected by circadian alignment (RER: $0.83 \pm 0.01$ vs. $0.84 \pm 0.01$, control vs. misalignment $p=0.687)$. We also determined resting energy expenditure 15 min after subjects woke up at either 7 AM or 7 PM in the control and misalignment condition respectively. Although mean energy expenditure again seemed to be higher upon circadian misalignment, this effect did not reach statistical significance (EE: $5.09 \pm 0.17$ vs. $5.35 \pm 0.16 \mathrm{~kJ} / \mathrm{min}$, control vs. misalignment $p=$ 0.14). Carbohydrate oxidation (0.162 \pm 0.014 vs. $0.152 \pm 0.022 \mathrm{~g} \mathrm{~min}^{-1}$, control vs. misalignment $p=0.707)$ and fat oxidation $(0.065 \pm 0.006$ vs. $0.076 \pm 0.009 \mathrm{~g} / \mathrm{min}$, control vs. misalignment $p=0.31$ ) were not affected by circadian misalignment.

\section{Mitochondrial oxidative capacity is elevated upon circadian misalignment}

Skeletal muscle is one of the determinants of resting metabolic rate and we recently showed day-night rhythmicity in mitochondrial function [8]. Therefore, we measured ex vivo mitochondrial oxidative capacity using high-resolution respirometry in isolated permeabilized muscle fibers in biopsies obtained at 7 AM and 7 PM in both control condition and circadian misalignment. ADP-stimulated mitochondrial respiration (State 3) upon octanoylcarnitine as a substrate did not show morningevening variation, neither in the control nor in the misalignment condition (state 3MO: $43.1 \pm 3.8$ and $43.3 \pm 3.7 \mathrm{pmol} / \mathrm{mg}^{*} \mathrm{~s}$ in control condition at $7 \mathrm{AM}$ and $7 \mathrm{PM}, \mathrm{p}$ $=0.925$ and $44.6 \pm 3.5$ and $45.6 \pm 3.5 \mathrm{pmol} / \mathrm{mg}^{*} \mathrm{~s}$ in misaligned condition at $7 \mathrm{AM}$ and $7 \mathrm{PM}, \mathrm{p}=0.674$, figure $4 \mathrm{~A}$ ). 
A

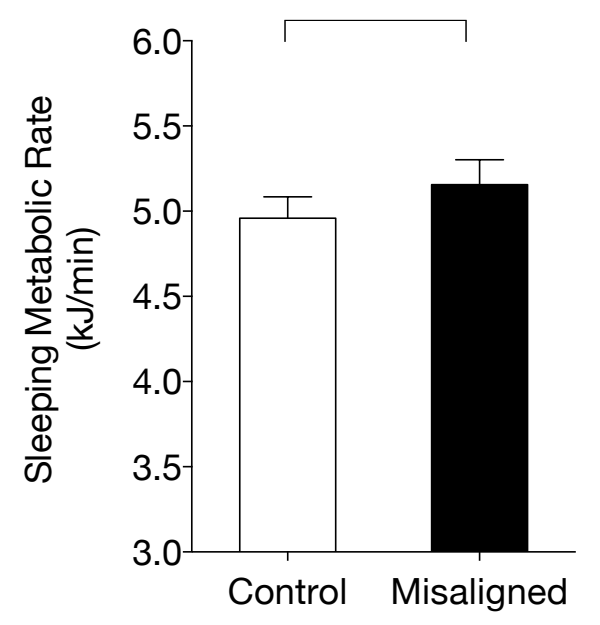

C

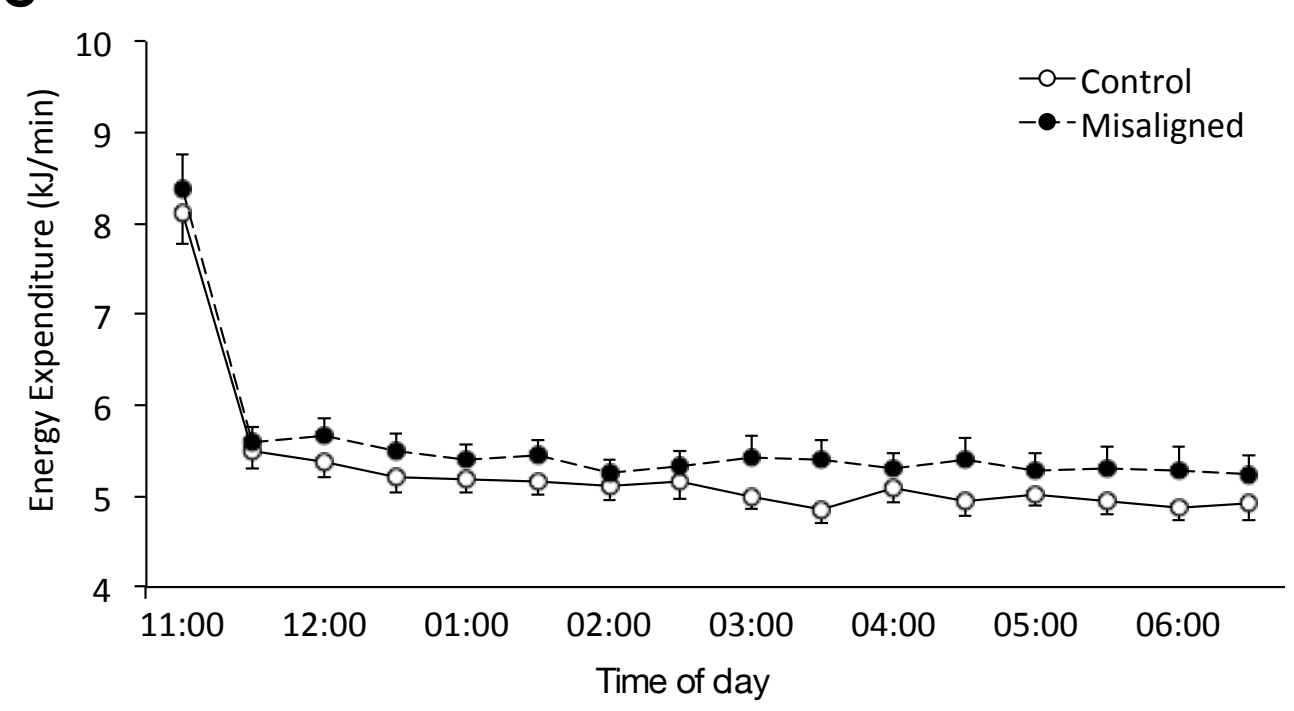

Figure 3: Sleeping Metabolic Rate (SMR) is increased in circadian misalignment Sleeping metabolic rate was measured in a whole-body room calorimeter and defined as the lowest energy expenditure during sleep during three consecutive hours (A). Respiratory Exchange Ratio (RER) during the same period is depicted in (B). As can be seen in (C), the increased metabolic rate upon circadian misalignment was present during the entire sleeping period. Data is expressed as mean \pm SEM. ${ }^{*} p<$ 0.05 
However, when the glycolytic metabolite pyruvate was used as a substrate, state 3 respiration showed higher respiration at $7 \mathrm{PM}$ compared to $7 \mathrm{AM}$ in the circadian misalignment condition (state 3MP: $69.3 \pm 4.3$ and $78.9 \pm 5.8 \mathrm{pmol} / \mathrm{mg} * \mathrm{~s}$, misaligned condition at $7 \mathrm{AM}$ and $7 \mathrm{PM}, \mathrm{p}=0.015$, figure 4) but not in the control condition (state 3MP: $65.6 \pm 4.4$ and $71.0 \pm 5.1 \mathrm{pmol} / \mathrm{mg}^{*} \mathrm{~s}$ in control condition at $7 \mathrm{AM}$ and 7 $\mathrm{PM}, \mathrm{p}=0.198$, figure $4 \mathrm{~B}$ ). As a result, state 3 respiration was higher upon circadian misalignment when measured in the 'overnight' fasted state (state 3 MP: $p=0.034$ ). To test if overall mitochondrial copy number or density affected these results, we measured total protein expression of mitochondrial electron transport chain complexes, which were not statistically different $(4.95 \pm 0.42$ and $4.94 \pm 0.34 \mathrm{RU}, 7$ $\mathrm{AM}$ and $7 \mathrm{PM}$ vs. $5.03 \pm 0.51$ and $5.29 \pm 0.49 \mathrm{RU}, 7 \mathrm{AM}$ and $7 \mathrm{PM}$, control vs. misalignment $p \geq 0.05$ )

\section{Fasting plasma metabolites are altered in circadian misalignment}

We next investigated the effect of circadian misalignment on overnight fasted clinically relevant plasma values. Thus, plasma metabolites were measured after an overnight fast for 10 hours either at 7 AM or 7 PM during the control condition and circadian misalignment respectively (figure 5). Glucose levels were significantly increased upon circadian misalignment $(5.0 \pm 0.1$ vs. $5.2 \pm 0.1 \mathrm{mmol} / \mathrm{l}$, control vs. misalignment $p=0.015)$, while insulin levels were not statistically changed (12.0 \pm 1.1 vs. $10.9 \pm 1.0 \mu \mathrm{U} / \mathrm{ml}$, control vs. misalignment $\mathrm{p}=0.209$ ). Plasma FFA levels were markedly increased upon circadian misalignment $(389 \pm 47$ vs. $525 \pm 48 \mu \mathrm{mol} / \mathrm{l}$, control vs. misalignment $p=0.006)$ whereas plasma triglyceride levels were decreased ( $0.76 \pm 0.05$ vs. $0.60 \pm 0.04 \mathrm{mmol} / \mathrm{l}$, control vs. misalignment $p=0.004)$.

\section{Insulin sensitivity is decreased in circadian misalignment}

We next investigated if the changes in plasma values upon circadian misalignment were due to a reduction in insulin sensitivity. To this end, we performed a 2-step hyperinsulinemic euglycemic clamp after an overnight fast, either at 8 AM or 8 PM in the control and misaligned condition respectively. We infused labeled 6-6-D2 tracer glucose to distinguish the relative contribution of liver and peripheral (mainly muscle) insulin sensitivity. 
A

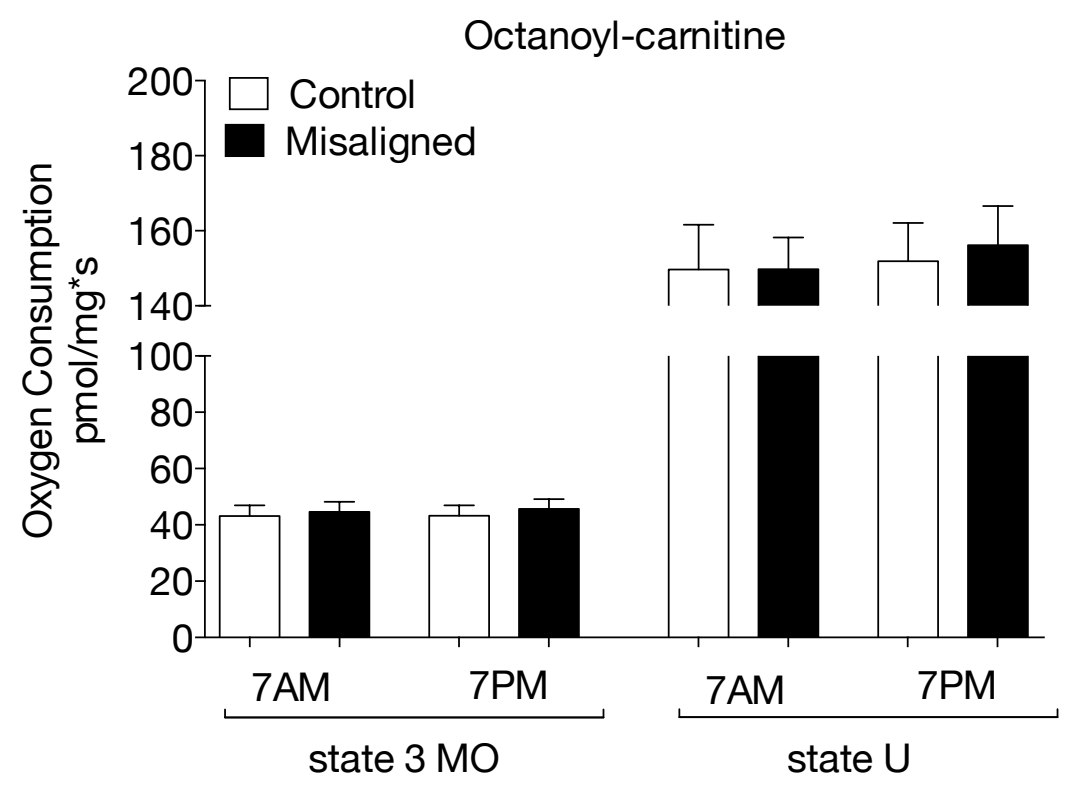

B

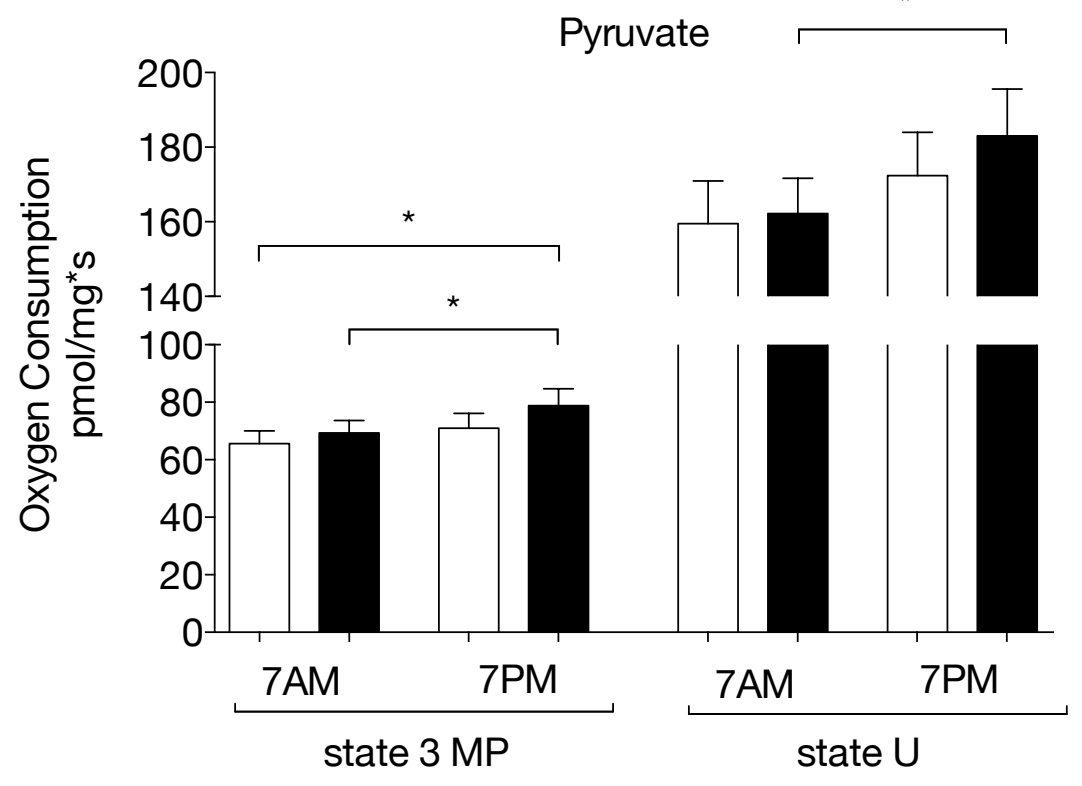

Figure 4: Mitochondrial oxidative capacity at 7AM and 7PM.

ADP stimulated mitochondrial respiration (state 3 ) and maximal uncoupled (state $U$ ) respiration in isolated permeabilized muscle fibers upon octanoylcarnitine (A) or pyruvate $(B)$ as a substrate. $M$, malate; $O$, octanoylcarnitine; $P$, pyruvate. Data represents oxygen consumption rates per $\mathrm{mg}$ wet weight per second, depicted as mean $\pm S E M .{ }^{*} p<0.05$. 
Circadian misalignment had no effect on endogenous glucose production (EGP) upon low dose insulin stimulation (EGP: $2.2 \pm 0.3$ vs. $2.4 \pm 0.2 \mu \mathrm{mol} / \mathrm{kg}^{*} \mathrm{~min}$, control vs. misalignment $p=0.424$ figure 6 B), nor on the percentage suppression of EGP, suggesting that circadian misalignment had no effect on hepatic insulin sensitivity. In line, during high insulin infusion, EGP was fully suppressed in both study conditions again suggesting similar hepatic insulin sensitivity. In contrast, however, circadian misalignment resulted in a $\sim 14 \%$ lower peripheral insulin sensitivity - as determined by the insulin stimulated glucose disposal - during high insulin infusion (delta $R_{d}$ : $35.2 \pm 2.4$ vs. $30.3 \pm 1.3 \mu \mathrm{mol} / \mathrm{kg}^{*} \min$, control vs. misalignment $p=0.029$, figure 6 A). The decrease in insulin-stimulated glucose uptake upon circadian misalignment was not accounted for by a reduced insulin-stimulated glucose oxidation (delta glucose oxidation: $11.2 \pm 0.8$ vs. $11.9 \pm 1.1 \mu \mathrm{mol} / \mathrm{kg}^{*} \mathrm{~min}$, control vs. misalignment, $\mathrm{p}$ $=0.583$, figure $6 \mathrm{C}$ ). However, insulin stimulated non-oxidative glucose disposal mainly accounted for by glycogen storage - was $23 \%$ lower upon circadian misalignment, suggesting decreased ability to form glycogen (delta NOGD: $23.7 \pm 2.4$ vs. $18.4 \pm 1.4 \mu \mathrm{mol} / \mathrm{kg}^{*} \min$, control vs. misalignment $\mathrm{p}=0.024$, figure $6 \mathrm{D}$ ).

Since FFA levels were increased during fasting conditions upon circadian misalignment, we also determined adipose tissue insulin sensitivity by measuring insulin-induced suppression of lipolysis during the clamp. First, FFA levels during the basal section of the clamp (first 2.5 hours, no insulin infusion) were indeed elevated in circadian misalignment compared to control condition (386 \pm 36 vs. $521 \pm 43$ $\mu \mathrm{mol} /$ I control vs. misalignment $\mathrm{p}<0.001$ ). Upon low and high dose insulin infusion, fatty acid levels decreased and were not different between control and misalignment condition (low insulin infusion: $74 \pm 11$ vs. $81 \pm 12 \mu \mathrm{mol} / \mathrm{l}$, control vs. misalignment $p=0.422$; high insulin infusion: $23 \pm 2$ vs. $26 \pm 3 \mu \mathrm{mol} / \mathrm{l}$, control vs. misalignment $p=0.456$ ), suggesting that circadian misalignment did not negatively affect adipose tissue insulin sensitivity.

Taken together, these results indicate that circadian misalignment results in an impairment of peripheral insulin sensitivity, which can be accounted for by a reduced capacity for non-oxidative glucose disposal. 
A

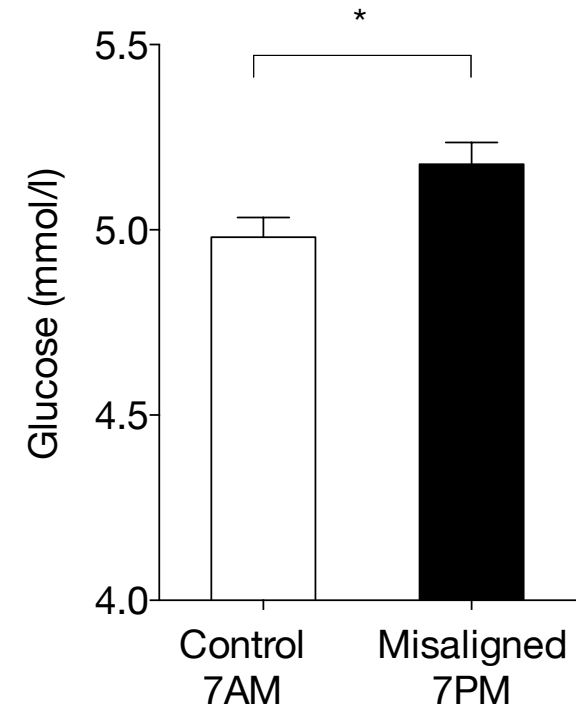

C

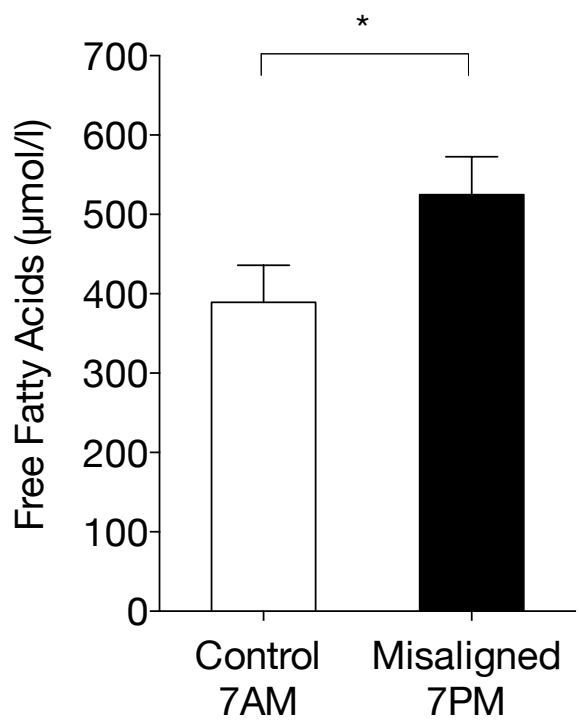

B
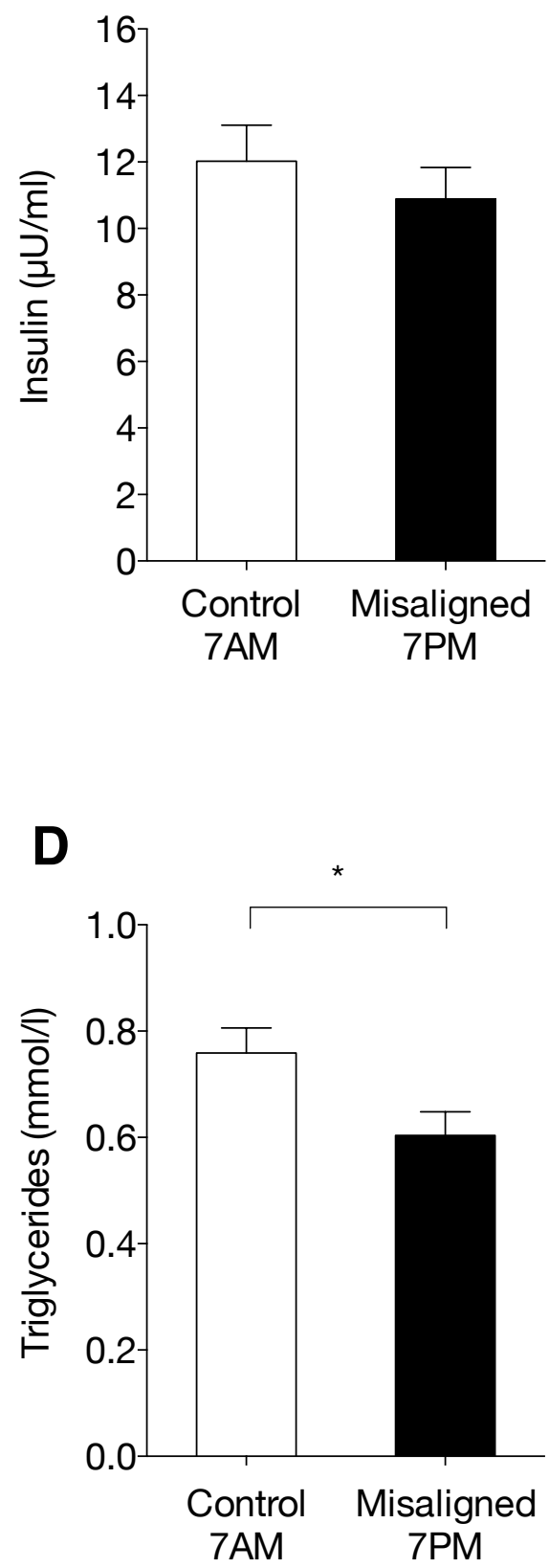

Figure 5: Plasma metabolites are altered in circadian misalignment

Plasma levels of glucose $(A)$, Insulin $(B)$, free fatty acids $(C)$ and triglycerides $(D)$ were measured in the 'overnight' fasted state 15 min after awaking at 7 AM or 7 PM in the control and misalignment condition respectively. Data are expressed as mean \pm SEM. * $p<0.05 ;{ }^{* *} p<0.01$. 
A

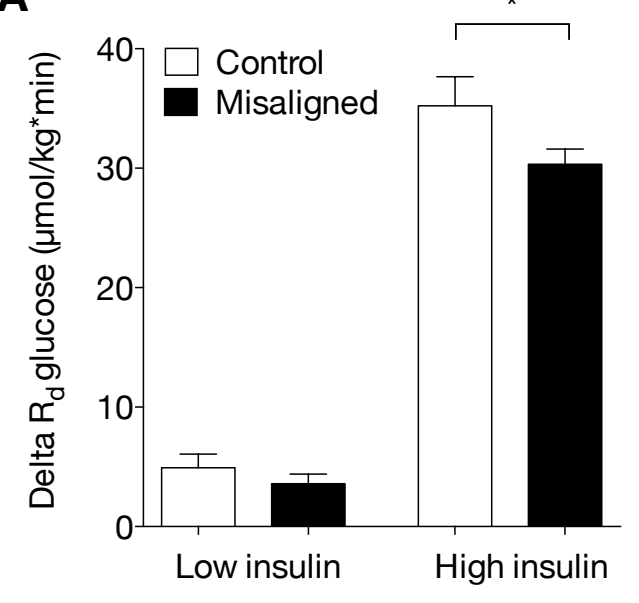

C

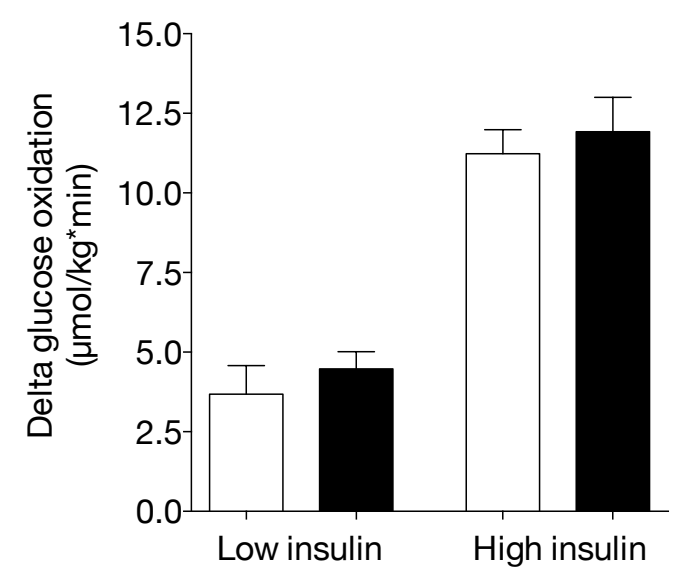

B

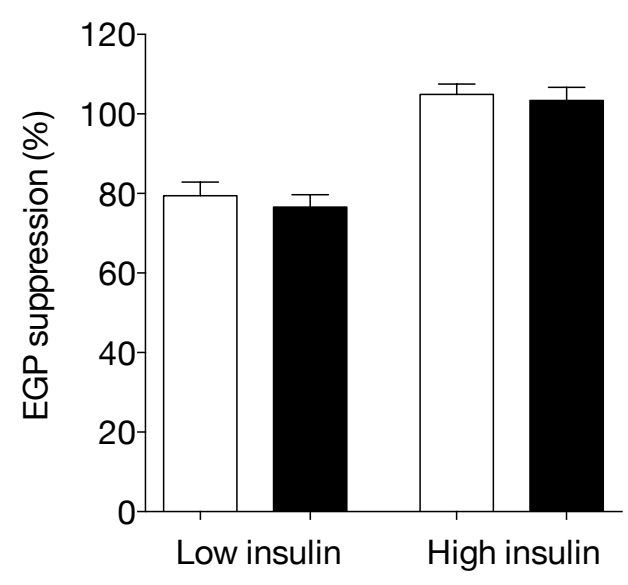

D

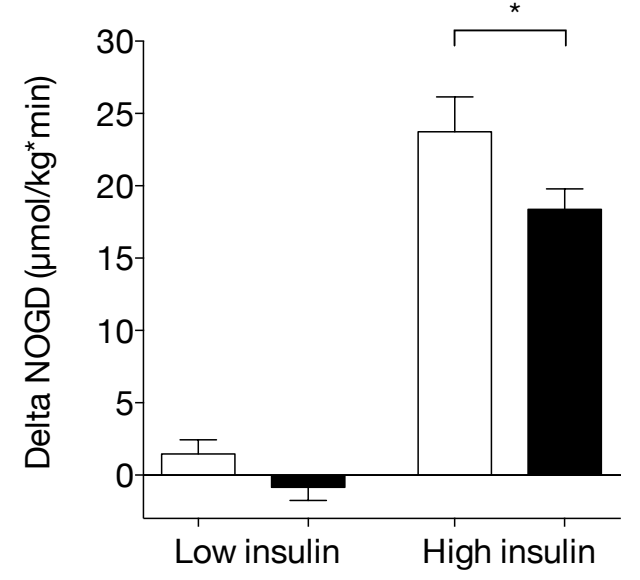

Figure 6: Insulin sensitivity is decreased in circadian misalignment

Insulin-stimulated glucose disposal is expressed as $R_{d}$ low insulin - $R_{d}$ basal (left) and $R_{d}$ high insulin - $R_{d}$ basal (A). Endogenous glucose production (EGP) suppression during low and high insulin infusion steady state is depicted in (B). Oxidative glucose disposal (C) and non-oxidative glucose disposal (NOGD) were corrected for basal values. All values were calculated for the last $30 \mathrm{~min}$ of the basal, low and high insulin steady states. Data are presented as mean \pm SEM. $R_{d}$, rate of disappearance. * $p<0.05$. 


\section{Core molecular clock of skeletal muscle does not adapt rapidly to 12-hour shifted} behavior

It has been shown that peripheral tissues have their own molecular clock that may be involved in the regulation of intrinsic energy metabolism. Since circadian misalignment affected peripheral insulin sensitivity, which is mainly accounted for by skeletal muscle, we next investigated if the core molecular clock showed disturbed circadian rhythmicity. To this end, we took two muscle biopsies: one was taken 12 hours before and the other one directly before the hyperinsulinemic-euglycemic clamp (control condition: 7 PM and 7 AM of the next day; misalignment condition: 7 $\mathrm{AM}$ and $7 \mathrm{PM}$ of the same day). We have previously shown that also in humans the core molecular clock components show day-night rhythms in skeletal muscle [8]. Therefore, we first determined if the core molecular clock components were disturbed upon circadian misalignment. In the control condition, the core clock genes BMAL1, CRY1 and PER2 displayed diurnal differences in mRNA expression levels, concordant with our previous study [8] (figure 7). Intriguingly, upon circadian misalignment, mRNA expression levels of the core clock genes kept the same diurnal pattern as in control condition, suggesting that the core molecular clock machinery in skeletal muscle was driven by circadian rhythmicity and did not align with the new day-night rhythm. We next investigated if this effect was only specific for core molecular clock genes. Therefore, we measured mRNA levels of representative key metabolic genes: whereas some genes displayed unchanged mRNA expression levels, other genes (i.e. HKII) were mainly driven by circadian rhythmicity, while a third category of regulatory genes (i.e. IRS1), displayed expression levels that seemed to be aligned with the new behavioral schedule (figure 8).

\section{Discussion}

A substantial part of the working population is engaged in shift-work, which affects the normal day-night rhythm and is associated with, amongst others, metabolic diseases. Indeed, animal studies have shown that disruption of the molecular clock leads to metabolic aberrations among others in skeletal muscle and results in insulin resistance. So far, it is unknown if circadian disruption affects muscle metabolism 
A
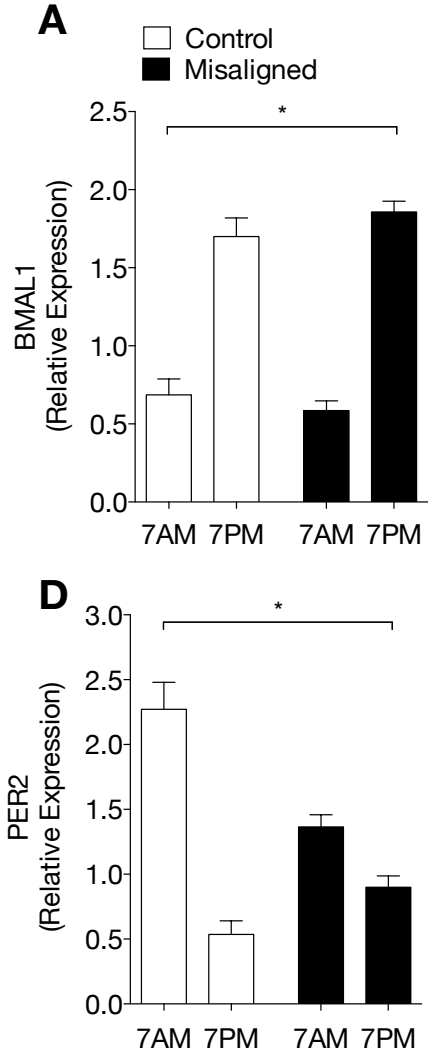

B
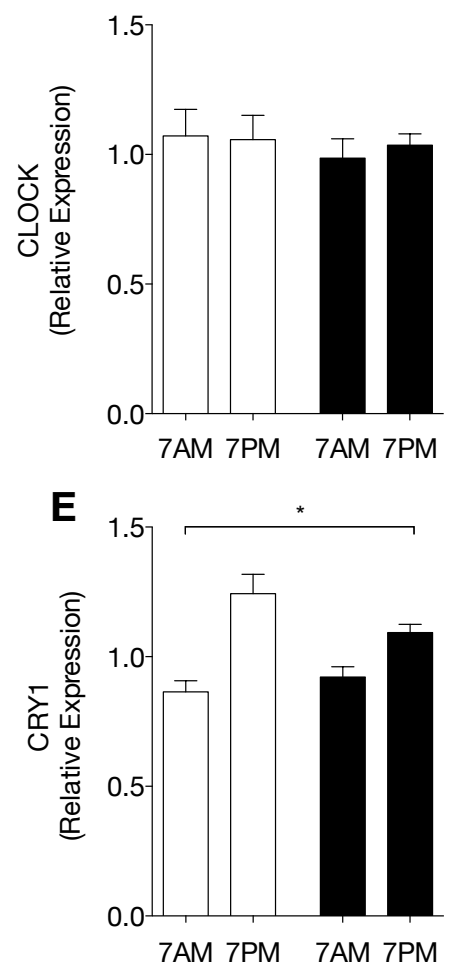

C

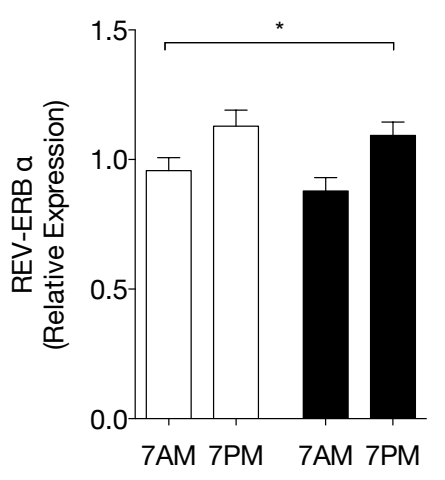

Figure 7: Core molecular clock genes are not adapted to behavioral rhythm upon circadian misalignment

mRNA expression levels of the core molecular clock genes BMAL1 (A), CLOCK (B), REV-ERB $\alpha(C), P E R 2(D)$ and CRY1 (E) in skeletal muscle measured by RT-QPCR. Data are normalized to the geometric mean of 3 housekeeping genes and presented as mean \pm SEM. ${ }^{*} p<0.05$ in control vs circadian misalignment in the 'overnight' fasted state.

and insulin sensitivity in humans. Here, we studied the effects of controlled circadian misalignment on insulin sensitivity and on both whole-body and skeletal muscle mitochondrial metabolism. We show that short-term circadian misalignment resulted in a significant decrease in insulin sensitivity in healthy young subjects that was mainly due to impairment in insulin-stimulated non-oxidative glucose disposal but unaffected by hepatic insulin sensitivity. In addition, we show that the molecular biological clock and several other key metabolic genes in skeletal muscle were 

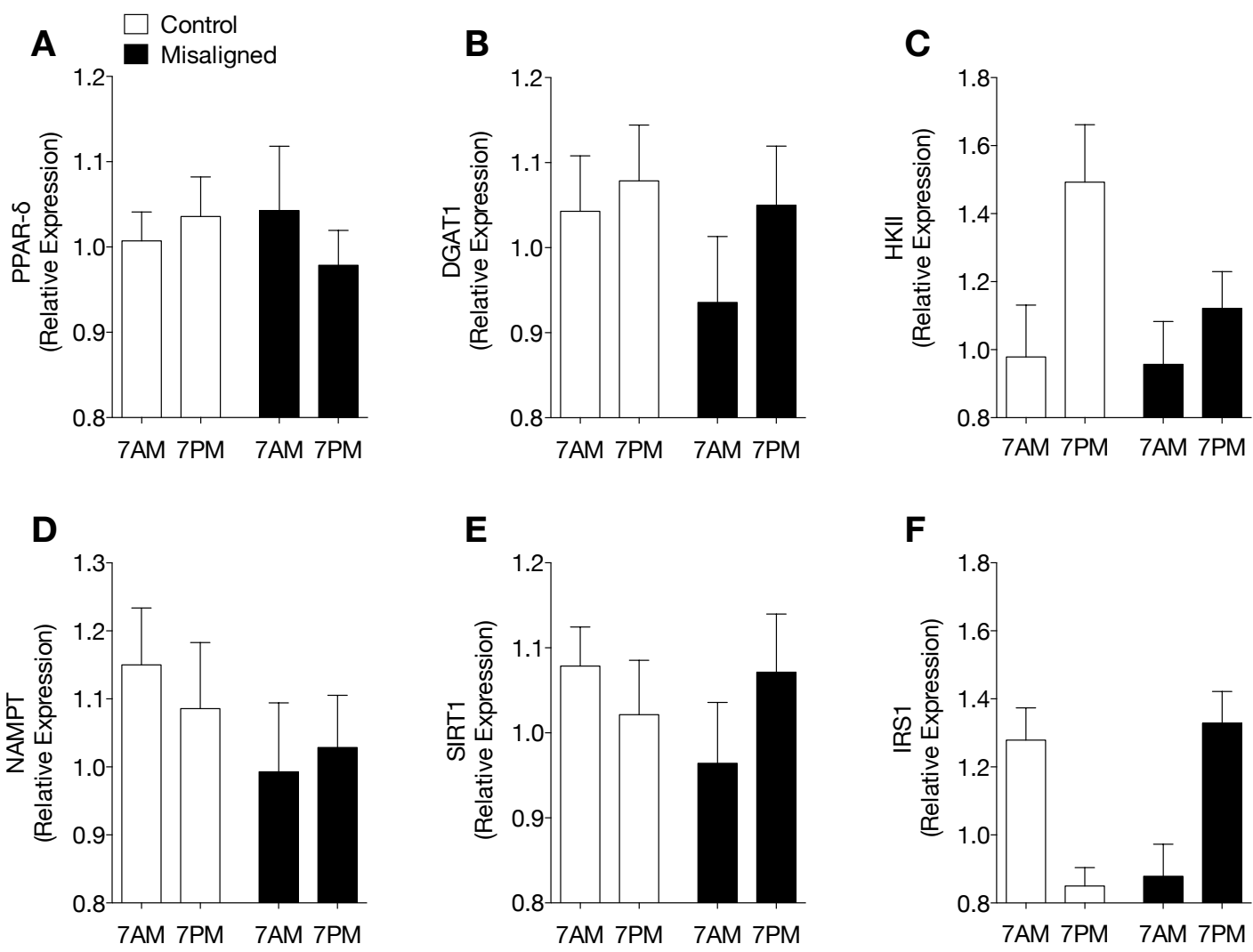

Figure 8: Metabolic genes expression levels upon circadian misalignment.

mRNA expression levels of the metabolic genes PPAR delta (A), DGAT1 (B), HKII (C), NAMPT (D) SIRT1 (E) and IRS1 (F) in skeletal muscle measured by RT-QPCR. Data are normalized to the geometric mean of 3 housekeeping genes and presented as mean \pm SEM. ${ }^{*} p<0.05$ in control vs circadian misalignment in the 'overnight' fasted state.

misaligned relative to the behavioral routine, which may underlie a disturbed metabolism. Interestingly and in contrast, another set of genes aligned rapidly to the new behavioral routine that was imposed by our study protocol. Together, these results show that circadian misalignment induced metabolic disturbances that can be detected at the molecular level in human skeletal muscle and may contribute to the risk for insulin resistance upon circadian misalignment.

Controlled circadian misalignment decreased insulin sensitivity - as measured by the gold standard hyperinsulinemic euglycemic clamp, and was accompanied by an 
increase in plasma glucose levels. This finding corroborates indirect observations from earlier studies, demonstrating higher plasma glucose levels after a meal, despite concomitantly increased levels of plasma insulin $[5,6]$ upon circadian misalignment. Interestingly, the decrease in insulin sensitivity under misaligned conditions was not due to a reduction in hepatic insulin sensitivity, as the suppression of hepatic glucose output upon physiological concentrations $\left(10 \mathrm{mU} / \mathrm{m}^{2} / \mathrm{min}\right)$ of insulin infusion was similar in both study conditions, indicating that hepatic insulin sensitivity is not affected during circadian misalignment. In fact, the decrease in insulin sensitivity could be completely attributed to a $23 \%$ decrease in insulin-stimulated non-oxidative glucose disposal (NOGD), compensated by a somewhat, but non-significant, higher insulin-stimulated glucose oxidation. The tendency towards higher glucose oxidation fits with the observation that mitochondrial ADP stimulated state 3 respiration upon pyruvate as a substrate was higher in circadian misalignment. Why non-oxidative glucose disposal is reduced upon circadian misalignment cannot be deduced from the current study. In healthy subjects, glucose taken up into skeletal muscle is mostly stored as glycogen [25], and it is therefore possible that already small alterations in pathways responsible for NOGD could explain the observed decrease.

In line with the reduced insulin sensitivity after circadian misalignment, we found a small, but significant increase in plasma glucose concentration after an 'overnight' fasting period in the misaligned condition compared to the control condition. Notably, elevated fasting glucose is an important diagnostic marker in the development of pre-diabetes and points towards a disturbed glucose homeostasis. Fasting insulin levels were not changed in circadian misalignment, which may suggest decreased responsiveness of the pancreatic beta-cells to increase insulin secretion to maintain glucose levels under the insulin-resistant conditions. Elevated fasting glucose could also originate from increased hepatic glucose output, but results obtained using the hyperinsulinemic euglycemic clamp did not point towards differences in either basal $\mathrm{Rd}$ or basal EGP between control and misaligned conditions. Apart from plasma glucose levels, circadian misalignment also lead to higher fasting FFA levels and lower triglyceride levels. Fasting FFA levels are mostly 
determined by lipolysis from adipose tissue, which is suppressed following insulin stimulation. We therefore measured FFA levels under fasting conditions immediately after awakening and again during the basal resting period of the insulin clamp. Of note, FFA levels remained markedly elevated $\sim 2.5$ hours after awakening. Nevertheless, insulin stimulated suppression of FFA was not impaired, suggesting that adipose tissue insulin sensitivity was not affected by circadian misalignment. To our knowledge this is the first study to measure adipose tissue insulin sensitivity in circadian misalignment in humans. These findings are of interest, since intrinsic circadian rhythmicity of adipose tissue lipolysis has been shown in mice [26]. Moreover, a recent human trial shows that the adipose tissue molecular clock responds to delayed meal timing with a shift in circadian phase [27]. The lower concentration of triglyceride levels in circadian misalignment cannot easily be explained. Since the majority of plasma triglycerides under fasting conditions are stored in VLDL particles that are secreted by the liver, an intrinsic circadian regulation seems possible. In fasted rats, VLDL levels are lower during the active phase compared to the resting phase [28], however, human data to support such regulation is lacking.

Together, these results highlight that the negative consequences of circadian misalignment extend beyond the reduction of insulin sensitivity and are visible under fasting conditions.

Circadian misalignment also resulted in a significantly higher sleeping metabolic rate (SMR) and a tendency for higher resting energy metabolism. These results are in line with previous reports of higher SMR after 3 days of circadian misalignment, induced by an artificial 27-h forced desynchrony protocol [29]. An elevation of energy metabolism may seem beneficial when regarded in the context of body weight regulation, but in general improvements in metabolic health are characterized by reductions in energy metabolism, due to improved energy efficiency. Therefore, the increase in sleeping metabolic rate is further proof for an unfavorable metabolic profile during circadian misalignment. 
Analysis of gene expression in skeletal muscle suggests that in circadian misalignment several genes were misaligned to the behavior of subjects, which might relate to the changes in metabolism. To the best of our knowledge, this is the first demonstration in humans that a short-term misalignment of 3 days is not enough to re-align the molecular clock in skeletal muscle and also affects other crucial metabolic genes. Hence, it is tempting to speculate that this may underlie the metabolic disturbances observed upon circadian misalignment. However, some other genes, for example IRS1 showed almost reversed expression patterns and thus was adapted to the new behavior cycle; this divergent effect of circadian misalignment on metabolic gene expression may result in misalignment of metabolic genes that under normal conditions may work together in regulating metabolic homeostasis. These results highlight the need to comprehensively study the effects of misalignment on skeletal muscle regulatory pathways by e.g. micro-arrays.

In conclusion, this study demonstrated that controlled circadian misalignment reduced insulin sensitivity, which supports the role of circadian disruption in the development of insulin resistance and T2DM. Furthermore, we showed that the molecular clock in skeletal muscle is affected by circadian misalignment. From a clinical perspective, fasting plasma glucose, FFA and resting energy requirements are elevated in circadian misalignment, demonstrating adverse metabolic consequences in healthy subjects. Future studies should assess whether similar results can be found in individuals at risk for the development of T2DM. 


\section{References}

[1] Buchvold, H.V., S. Pallesen, N.M. Oyane, and B. Bjorvatn, 2015. Associations between night work and BMI, alcohol, smoking, caffeine and exercise--a cross-sectional study. BMC Public Health 15(1112.

[2] Gan, Y., C. Yang, X. Tong, H. Sun, Y. Cong, X. Yin, et al., 2015. Shift work and diabetes mellitus: a meta-analysis of observational studies. Occup Environ Med 72(1):72-78.

[3] Karlsson, B., A. Knutsson, and B. Lindahl, 2001. Is there an association between shift work and having a metabolic syndrome? Results from a population based study of 27,485 people. Occup Environ Med 58(11):747752.

[4] Leproult, R., U. Holmback, and E. Van Cauter, 2014. Circadian misalignment augments markers of insulin resistance and inflammation, independently of sleep loss. Diabetes 63(6):1860-1869.

[5] Morris, C.J., J.N. Yang, J.I. Garcia, S. Myers, I. Bozzi, W. Wang, et al., 2015. Endogenous circadian system and circadian misalignment impact glucose tolerance via separate mechanisms in humans. Proc Natl Acad Sci U S A 112(17):E2225-2234.

[6] Scheer, F.A., M.F. Hilton, C.S. Mantzoros, and S.A. Shea, 2009. Adverse metabolic and cardiovascular consequences of circadian misalignment. Proc Natl Acad Sci U S A 106(11):4453-4458.

[7] Eckel-Mahan, K.L., V.R. Patel, S. de Mateo, R. Orozco-Solis, N.J. Ceglia, S. Sahar, et al., 2013. Reprogramming of the circadian clock by nutritional challenge. Cell 155(7):1464-1478.

[8] van Moorsel, D., J. Hansen, B. Havekes, F.A. Scheer, J.A. Jorgensen, J. Hoeks, et al., 2016. Demonstration of a day-night rhythm in human skeletal muscle oxidative capacity. Mol Metab 5(8):635-645.

[9] Kumar Jha, P., E. Challet, and A. Kalsbeek, 2015. Circadian rhythms in glucose and lipid metabolism in nocturnal and diurnal mammals. Mol Cell Endocrinol 418 Pt 1(74-88.

[10] Andrews, J.L., X. Zhang, J.J. McCarthy, E.L. McDearmon, T.A. Hornberger, B. Russell, et al., 2010. CLOCK and BMAL1 regulate MyoD and are necessary for maintenance of skeletal muscle phenotype and function. Proc Natl Acad Sci U S A 107(44):19090-19095.

[11] Peek, C.B., A.H. Affinati, K.M. Ramsey, H.Y. Kuo, W. Yu, L.A. Sena, et al., 2013. Circadian clock NAD+ cycle drives mitochondrial oxidative metabolism in mice. Science 342(6158): 1243417.

[12] Dyar, K.A., S. Ciciliot, L.E. Wright, R.S. Bienso, G.M. Tagliazucchi, V.R. Patel, et al., 2014. Muscle insulin sensitivity and glucose metabolism are controlled by the intrinsic muscle clock. Mol Metab 3(1):29-41.

[13] Jacobi, D., S. Liu, K. Burkewitz, N. Kory, N.H. Knudsen, R.K. Alexander, et al., 2015. Hepatic Bmal1 Regulates Rhythmic Mitochondrial Dynamics and Promotes Metabolic Fitness. Cell Metab 22(4):709-720.

[14] Fabbri, E., C.W. Chia, R.G. Spencer, K.W. Fishbein, D.A. Reiter, D. Cameron, et al., 2017. Insulin Resistance Is Associated With Reduced Mitochondrial Oxidative Capacity Measured by 31P-Magnetic Resonance Spectroscopy in Participants Without Diabetes From the Baltimore Longitudinal Study of Aging. Diabetes 66(1):170-176.

[15] Phielix, E., V.B. Schrauwen-Hinderling, M. Mensink, E. Lenaers, R. Meex, J. Hoeks, et al., 2008. Lower intrinsic ADP-stimulated mitochondrial respiration underlies in vivo mitochondrial dysfunction in muscle of male type 2 diabetic patients. Diabetes 57(11):2943-2949. 
[16] Peronnet, F. and D. Massicotte, 1991. Table of nonprotein respiratory quotient: an update. Can J Sport Sci 16(1):23-29.

[17] Weir, J.B., 1949. New methods for calculating metabolic rate with special reference to protein metabolism. J Physiol 109(1-2):1-9.

[18] Schoffelen, P.F. and K.R. Westerterp, 2008. Intra-individual variability and adaptation of overnight- and sleeping metabolic rate. Physiol Behav 94(2):158-163.

[19] Bergstrom, J., L. Hermansen, E. Hultman, and B. Saltin, 1967. Diet, muscle glycogen and physical performance. Acta Physiol Scand 71(2):140-150.

[20] Hoeks, J., N.A. van Herpen, M. Mensink, E. Moonen-Kornips, D. van Beurden, M.K. Hesselink, et al., 2010. Prolonged fasting identifies skeletal muscle mitochondrial dysfunction as consequence rather than cause of human insulin resistance. Diabetes 59(9):2117-2125.

[21] Hansen, J., S. Timmers, E. Moonen-Kornips, H. Duez, B. Staels, M.K. Hesselink, et al., 2016. Synchronized human skeletal myotubes of lean, obese and type 2 diabetic patients maintain circadian oscillation of clock genes. Sci Rep 6(35047.

[22] DeFronzo, R.A., J.D. Tobin, and R. Andres, 1979. Glucose clamp technique: a method for quantifying insulin secretion and resistance. Am $\mathrm{J}$ Physiol 237(3):E214-223.

[23] Mensink, M., E.E. Blaak, M.A. van Baak, A.J. Wagenmakers, and W.H. Saris, 2001. Plasma free Fatty Acid uptake and oxidation are already diminished in subjects at high risk for developing type 2 diabetes. Diabetes 50(11):25482554.

[24] Steele, R., 1959. Influences of glucose loading and of injected insulin on hepatic glucose output. Ann N Y Acad Sci 82(420-430.

[25] Shulman, G.I., D.L. Rothman, T. Jue, P. Stein, R.A. DeFronzo, and R.G. Shulman, 1990. Quantitation of muscle glycogen synthesis in normal subjects and subjects with non-insulin-dependent diabetes by $13 \mathrm{C}$ nuclear magnetic resonance spectroscopy. N Engl J Med 322(4):223-228.

[26] Shostak, A., J. Meyer-Kovac, and H. Oster, 2013. Circadian regulation of lipid mobilization in white adipose tissues. Diabetes 62(7):2195-2203.

[27] Wehrens, S.M.T., S. Christou, C. Isherwood, B. Middleton, M.A. Gibbs, S.N. Archer, et al., 2017. Meal Timing Regulates the Human Circadian System. Curr Biol 27(12):1768-1775 e1763.

[28] Mondola, P., P. Gambardella, F. Santangelo, M. Santillo, and A.M. Greco, 1995. Circadian rhythms of lipid and apolipoprotein pattern in adult fasted rats. Physiol Behav 58(1):175-180.

[29] Gonnissen, H.K., F. Rutters, C. Mazuy, E.A. Martens, T.C. Adam, and M.S. Westerterp-Plantenga, 2012. Effect of a phase advance and phase delay of the $24-\mathrm{h}$ cycle on energy metabolism, appetite, and related hormones. Am J Clin Nutr 96(4):689-697. 


\section{CHAPTER 7}

General discussion and future perspectives 


\section{General discussion and future perspectives:}

Through the last century, changes in social- and economic status together with technological advance such as artificial light and fridges have enabled humans to disengage from their primordial day-night rhythm. Obviously, this comes not without risks. Since 1980 the obesity prevalence has more than doubled in over 70 countries [1], while the number of T2D diagnoses almost quadrupled [2]. This development entails increased risks for obesity related comorbidities, ultimately resulting in shortened burden free lifetime and overall life expectancy $[1,3]$. Further, epidemiological studies indicate that the recent lifestyle changes contribute to a disruption of day-night rhythm, which has been associated with negative consequences for metabolic health [4-7]. Unravelling by which mechanism this disruption contributes to derangements in metabolic health may be of great value to strengthen current prevention and treatment strategies. Therefore, this thesis focusses on investigating the role of the biological clock in human energy metabolism and the putative involvement of peripheral rhythms in the etiology of obesity and T2D.

\section{Do human peripheral tissues possess a molecular clock?}

The presence of circadian rhythms is a fundamental characteristic of living cells. From single cell to multicellular organisms behavioral and physiological rhythms are present. While there is broad understanding in how organs interact in mammalian physiology, it is less well known how rhythms are involved in cellular and whole body homeostasis throughout repetitive day and night cycles.

According to our current understanding, the mammalian timing system comprises of a central clock - situated in the suprachiasmatic nucleus (SCN) of the hypothalamus - this clock orchestrates the temporal organization of behavioral, physiological, neuronal and cellular processes. Additional peripheral molecular clocks are assumed to be maintained in virtually every cell of the organism, allowing cells to individually keep track of time. To result in temporal homeostasis, such a peripheral molecular clock consists of an autonomous but adaptable mechanism, that facilitates cellular time keeping to allow peripheral organs to resonate in synchrony. This mechanism is explained in detail in CHAPTER 3.

When starting this thesis project, data on rhythms in human peripheral tissues was limited to - rather accessible - skin and blood cells and white adipocytes. Especially for 
metabolically important tissues that are not readily accessible like pancreas, liver and skeletal muscle, data had not yet been available. Hitherto, findings from animal studies pointed towards an intimate link between peripheral molecular clocks and whole-body metabolism regulation. In this respect, we had hypothesized that healthy human skeletal muscle also possesses a circadian molecular clock to contribute to temporal homeostasis. Hence, as reported in CHAPTER 2, we developed and tested a cellular model that employed serum shock to result in synchronization of cultured human primary myotubes. This model's particular value is that cells with the donor's genetic and possibly epigenetic background can be observed outside of their (patho-)physiological milieu. Hence, rhythms occurring in such isolation can be considered truly intrinsic. Identifying whether a particular rhythm is of intrinsic or behavioral origin is of great value as it increases our understanding by which factors human time keeping is driven. Indeed, intrinsic circadian rhythms were sustained in synchronized human skeletal myotubes for at least 72 hours (CHAPTER 3). Interestingly, although nutritional supply and other culture conditions were kept constant, expression of the cellular energy sensors - SIRT1 and REVERBA - was also intrinsically rhythmic. This is of particular interest, as these sensors are thought to be part of a bilateral regulatory intersection between the molecular clock and cellular energy metabolism. In parallel, another cell study published concordant circadian rhythms of the molecular clock, using different agents for synchronization - forskolin and dexamethasone [8]. Together, the findings of CHAPTER 3 and Perrin et al. [8], indicate that circadian rhythms were relevant in human skeletal muscle function. Therefore, in CHAPTER 5 it was explored, whether 24-hour rhythms were also present in serial human skeletal muscle biopsies and whole body metabolism. Indeed, in vivo human skeletal muscle also sustains 24-hour rhythms in molecular clock gene expression under normal behavioral cycles.

These findings are in line with extensive animal studies underpinning that mammalian peripheral tissues possess a circadian molecular clock in virtually every tissue: heart [9], pancreas [10], liver [11], white adipose tissue [12] skeletal muscle [13] and many more.

\section{What is the role of the biological clock in metabolic health?}

The biological clock orchestrates cellular function, some of which are tissue specific Segregating molecular processes spatially and temporally is fundamental to prosper in an intermittently changing milieu. For example, the concurrent activation of glycogen depletion 
and its re-synthesis in skeletal muscle metabolism, would result in futile cycles. Hence, the muscle molecular clock is a governing mechanism for anticipating recurring phases. Indeed, in CHAPTER 3 it was demonstrated that metabolic key-regulators can be intrinsically rhythmic in synchronized muscle cells of healthy donors. This signalizes that human skeletal muscle intrinsically anticipates diurnal fast-fed states on a cellular level.

In parallel to this project, other in vitro studies were published reporting that the peripheral molecular clock governs cellular functions that are relevant in metabolic health. For instance, Perrin et al. [8] found CLOCK-dependent temporal regulation in myokine release in synchronized human myoblasts, while Peek et al. [14] reported intrinsic rhythmicity of mitochondrial function in mouse myotubes. In line with this, Feneberg et al. [15] found rhythms in insulin sensitivity in synchronized rat myotubes. Clearly, these studies together with CHAPTER 3 concert in demonstrating that the molecular clock is central in orchestrating temporal tissue specific cell function. Noteworthy, as described in CHAPTER 5, the human skeletal muscle biopsy analysis of one exemplary participant confirmed that 14.5 $\%$ of all expressed transcripts were rhythmic. This is concordant with findings from animal circadian rhythm studies; it is generally accepted that in any given mouse tissue approximately $10 \%$ of the transcriptome is under circadian control. Obviously, when comparing transcriptomic analysis of different organs, the ratio of rhythmic transcripts can vary largely, possibly also due to methodological and model differences. Still, it has been concluded in a comparative analysis that more than $43 \%$ of all protein coding transcripts are rhythmic in one or more organs [16]. Importantly, many but not all cyclic transcript result in rhythmically expressed proteins [17]. Interestingly, we found total protein content itself to be intrinsically rhythmic in synchronized human myotubes, as described in CHAPTER 4. In the light of posttranslational mechanisms the functional implication of cyclic proteins is even more complex and needs further investigation [18].

\section{Synchronizing all organs benefits temporal homeostasis.}

Producing single molecules transiently, and being able to switch on and off complete pathways to circumvent renewal of a molecular machinery in a next cycle, is a vast advantage for cellular physiology [19]. When considering this on a whole-body level, preparing an individual to a short time frame to result in absolute peak performance, rather 
than performing just well at all times, can in both cases provide a certain advance in natural selection.

A physiological example at which timing plays an important role is the day-night rhythm of substrate utilization. The central nervous system can exclusively utilize carbohydrates. With food consumption being halted during sleep, prudent carbohydrate metabolism can prevent excess utilization by peripheral organs. As displayed in CHAPTER 5, the body utilizes carbohydrates throughout the day. In turn, during sleep fat is predominantly oxidized until resuming food consumption in the morning. That these substrate utilization preferences underlie an anticipatory adaptation by peripheral organs is apparent when examining postprandial clearance of energy substrates: During day-time clearance of dietary fat did not result in an increased fat utilization, in fact it decreased progressively until midnight. Independent of circadian timing, the capacity to adjust substrate oxidation to alterations in substrate availability (e.g. during sleep and postprandial state), often referred to as metabolic flexibility, has been linked to metabolic health [20]. The capacity to adjust substrate oxidation to substrate supply is most likely also dependent on mitochondrial metabolism. Hence, we hypothesized in CHAPTER 5 that mitochondrial function in muscle of young healthy volunteers is rhythmic. Indeed, mitochondrial oxidative capacity displayed 24-hour oscillations with a maximal peak in the late evening. Interestingly, human exercise peak performance, which is largely dependent on mitochondrial function, has been reported to occur in the evening $[21,22]$. In terms of energy preservation as described in the supplementary section of CHAPTER 5 and in CHAPTER 6, core body temperature is dropping during sleep, which coincides with lowest energy consumption within 24 hours.

Against this background, it can already be suggested that human energy metabolism is strongly linked to temporal organization of peripheral organ function. In CHAPTER 6, we confirmed this suggestion by misaligning the biological clock with the behavioral cycles, as discussed in the next section.

\section{Disturbances of the biological clock cause acute and long-term metabolic derangement}

Prior to the study described in CHAPTER 6, it was established in animal studies that a disrupted peripheral clock in one or more organs can lead to substantial impairments of metabolic health. In part, this could be explained by employing gene perturbation studies, demonstrating that core clock genes can drive tissue specific function in vivo. For instance, 
the hepatic clock governs fasting glycemic control and glucose disposal [23], the pancreatic clock governs insulin release $[10,24,25]$, the adipose clock governs lipid storage and mobilization [26, 27] and the skeletal muscle clock governs glucose uptake and mitochondrial function [28, 29]. Long term, chronic disruption by global and peripheral clocks can severely impact glucose and lipid metabolism with peculiar manifestations for each clock gene, as reviewed in detail [30]. Ultimately, whole-body implications such as increased body weight [28], increased adiposity [31, 32] and also diabetic conditions [24, 33] have been observed.

These metabolic implications could be better understood when considering that temporal homeostasis is dependent on a hierarchic circadian timing system. In mice, it has been demonstrated by lesioning the SCN of mice that the SCN harbors the central clock [34, 35]. In fact, it was shown that the a transplanted SCN imposed the donor's circadian phase and behavioral cycles to the host mice [36]. When the central clock is defective, as in ablation of the SCN, mice lose their rhythmicity. Interestingly, independent of the SNC, the peripheral clock can also be entrained by behavioral rhythms such as restricted feeding [37] and scheduled exercise [38]. However, when forcing mice with an intact SCN to consume food out of their rhythm (i.e. resting phase), this led to overweight, while control mice fed at normal times with equal caloric intake remained at normal weight [26].

As common in shift work and other social jetlag conditions, such shift in behavioral rhythms against the circadian rhythm is defined as circadian misalignment, as discussed elaborately in CHAPTER 6. Previous human studies demonstrated that circadian misalignment led to impaired glucose control in healthy participants $[39,40]$. However, it could not be revealed, whether this was due to misalignment of human peripheral clocks, and whether insulin sensitivity per se was impaired. The data from CHAPTER 5 suggests that temporal organization is key in normal lifestyle energy homeostasis. Hence, in CHAPTER 6 a circadian misalignment protocol was used to create novel understanding about the metabolic implications on liver, muscle and whole-body level in healthy volunteers. Indeed, we found prominent anomalies of human metabolism in circadian misalignment. Peripheral misalignment was confirmed by inversed profiles of core molecular clock genes and by core body temperature after two days of misalignment, entailing strong disturbances in metabolic homeostasis. For instance, peripheral insulin sensitivity in circadian misalignment was reduced. This derangement occurred in parallel with elevated plasma levels of glucose, 
free fatty acids and lowered triglyceride levels. Similar observations on plasma levels were previously reported by others [39, 40]. Furthermore, sleeping metabolic rate was increased during circadian misalignment. Interestingly, mitochondrial oxidative capacity driven by pyruvate, was markedly increased in circadian misalignment, but not for metabolites of beta-oxidation.

Hence, with CHAPTER 6, we demonstrate overt metabolic implications that result from only 2 days of maximal circadian misalignment. Albeit not yet studied, it seems reasonable to suggest that (given the inflexibility of the central and molecular clock) such misalignment would persist throughout several days. Hence, implicating even greater risks for metabolic health. These findings may be highly relevant to people with recurring misalignment, such as in regular time-zone travelling and to people suffering from regular social jetlag, as in shift work.

\section{Could the biological clock be used as a target to improve prevention and treatment strategies for obesity and T2D?}

A frequent observation in the etiology of T2D is the association of progressively impaired mitochondrial function and reduced insulin sensitivity. In healthy human subjects, glucose metabolism is diurnal, with higher glucose tolerance [41, 42] and insulin sensitivity [43] in the morning compared to the evening. Similar findings have been reported when identical meal tests were used to determine markers of glucose tolerance $[44,45]$. These findings are supported by the 24-hour rhythm in glucose metabolism characterized in CHAPTER 5. In this chapter, we further demonstrate that healthy men do have a 24-hour rhythm in mitochondrial function. As shown in CHAPTER 6, circadian misalignment affected mitochondrial function and insulin sensitivity, both of which were demonstrated to rely on proper functioning of the molecular clock, as discussed above.

In CHAPTER 6, we used a 12-hour misalignment protocol, which is an extreme form of circadian misalignment. However, there are also other studies with less radical interventions, resulting in observations concordant to our findings. For instance, ambient light is an important time cue to the SCN. Interestingly, morning exposure to bright ambient light in type 2 diabetes patients, induced elevated fasting and postprandial glucose levels, but not in healthy controls [46]. This underscores the importance of the biological clock in 
glucose homeostasis and emphasizes the importance of artificial light conditions in home and nursing environment for long-term glucose control.

Further, as mentioned previously, adverse timing of food consumption e.g. scheduled at time of sleep can result in increased adiposity in animals [26, 47]. More importantly, as demonstrated in a human study, shifted timing of meals also results in modest circadian misalignment: Switching from early to late meals for several days resulted in a phase shift in the peripheral clock of white adipose tissue, whilst subjective hunger, sleepiness and central clock markers did not shift in phase [48]. Furthermore, eating late-lunch was associated to lower effectiveness of a 20-week weight-loss intervention [49]. In addition, also delaying sleep by 4 hours, without sleep loss, reduced rhythmicity in human blood transcriptome in a forced-desynchrony protocol [50]. These findings, together with the conclusions from CHAPTER 5 and 6, strengthen the assumption that the alignment of the behavioral cycle, as in food intake and activity, with the circadian clock is beneficial for metabolic health.

Another important aspect, which is of demographic and societal impact is that humans live by different chronotypes. Especially, a late chronotype was associated with a higher risk to develop obesity and T2D when compared to early chronotypes [51,52]. While chronotype can change throughout lifespan, there is also a genetic component in our biological clock. Amongst others, SNPs in core clock genes have been associated to weight-loss response [53], meal-time preference [49] and even type 2 diabetes risk [54].

Although not addressed in this thesis project, another important factor demands to be mentioned: dysbiosis. Obviously, discussion of the relation between microbiome and metabolic health $[55,56]$ are beyond the scope of this thesis, but data from animal studies indicates that the gut microbiota has a circadian phase [56]. Therefore, misalignment of host and gut microbiome phases can lead to dysbiosis [57], entailing metabolic implications, such as glucose intolerance and promote obesity $[56,57]$. Whether a putative dysbiosis would have relevant impact on human metabolism remains to be studied in the future.

Finally, in the present thesis, we further hypothesized, that disturbances in the makeover of the human molecular clock contribute to the pathophysiology of metabolic disease. Hence, in CHAPTER 3 we sought, whether muscle cells of obese and T2D patients were different in core clock gene expression and metabolic gene expression compared to healthy donors. While core clock genes were similarly rhythmic in all groups, we found dampened 
rhythmicity in expression of the cellular energy sensors - SIRT1 and REVERBA - in cells of obese and T2D.

SIRT1, is an intricate part of the AMPK-SIRT1-PGC1a axis, an intricate pathway that is central to cellular energy metabolism. Noteworthy, data published by Stenvers et al. [58] also report dampened rhythms for transcripts of the AMPK pathway in human white adipose tissue obtained from T2D patients. The role of this pathway is central in the regulation of metabolism [59], and it has been found that activation of this pathway - for example by food compound supplementation, drug treatment, calorie restriction and exercise - is a promising strategy for the prevention and treatment of metabolic diseases.

Furthermore, in CHAPTER 4, we found markedly impaired temporal mitochondrial function in myotubes of T2D, when compared to the other groups. It is important to note, that synchronization of T2D myotubes lead to robust rhythms in core clock genes and total protein content, but synchronization was not sufficient in rescuing impaired mitochondrial function and rhythms in some circadian genes. Hence, restoring robust rhythms may be certainly beneficial for metabolic health on whole-body scale, but cannot be considered to rescue mitochondrial dysfunction in T2D in this model.

In conclusion, the data presented in this thesis strengthens the current idea that the biological clock is an important risk factor to metabolic diseases. Clearly, the data from CHAPTER 5 and 6, together with the current literature, demonstrate that circadian misalignment triggers metabolic aberrations which in the long-run may result in metabolic disease. Hence, prevention and treatment strategies of circadian misalignment deserve to be considered as first-line treatment and prevention for metabolic disorders like obesity and type 2 diabetes. Thus, next to counting calories, also timing the consumption of food is essential to maintain metabolic health. Certainly, against the background of CHAPTER 3 and 4, there is still need to unravel the ramifications of the molecular makeover of the peripheral clocks and cellular metabolism in humans. Ultimately, chronotherapeutic approaches could be used to improve treatment efficacy in metabolic diseases. 


\section{References:}

[1] Collaborators, G.B.D.O., 2017. Health Effects of Overweight and Obesity in 195 Countries over 25 Years. N Engl J Med.

[2] Collaboration, N.C.D.R.F., 2016. Worldwide trends in diabetes since 1980: a pooled analysis of 751 population-based studies with 4.4 million participants. Lancet 387(10027):1513-1530.

[3] Global Burden of Metabolic Risk Factors for Chronic Diseases, C., 2014. Cardiovascular disease, chronic kidney disease, and diabetes mortality burden of cardiometabolic risk factors from 1980 to 2010: a comparative risk assessment. Lancet Diabetes Endocrinol 2(8):634-647.

[4] Pan, A., E.S. Schernhammer, Q. Sun, and F.B. Hu, 2011. Rotating night shift work and risk of type 2 diabetes: two prospective cohort studies in women. PLoS Med 8(12):e1001141.

[5] Suwazono, Y., M. Dochi, M. Oishi, K. Tanaka, E. Kobayashi, and K. Sakata, 2009. Shiftwork and impaired glucose metabolism: a 14-year cohort study on 7104 male workers. Chronobiol Int 26(5):926-941.

[6] Parsons, M.J., T.E. Moffitt, A.M. Gregory, S. Goldman-Mellor, P.M. Nolan, R. Poulton, et al., 2015. Social jetlag, obesity and metabolic disorder: investigation in a cohort study. Int J Obes (Lond) 39(5):842-848.

[7] Mattson, M.P., D.B. Allison, L. Fontana, M. Harvie, V.D. Longo, W.J. Malaisse, et al., 2014. Meal frequency and timing in health and disease. Proc Natl Acad Sci U S A 111(47):16647-16653.

[8] Perrin, L., U. Loizides-Mangold, S. Skarupelova, P. Pulimeno, S. Chanon, M. Robert, et al., 2015. Human skeletal myotubes display a cell-autonomous circadian clock implicated in basal myokine secretion. Mol Metab 4(11):834-845.

[9] Durgan, D.J., N.A. Trexler, O. Egbejimi, T.A. McElfresh, H.Y. Suk, L.E. Petterson, et al., 2006. The circadian clock within the cardiomyocyte is essential for responsiveness of the heart to fatty acids. J Biol Chem 281(34):24254-24269.

[10] Peschke, E. and D. Peschke, 1998. Evidence for a circadian rhythm of insulin release from perifused rat pancreatic islets. Diabetologia 41(9):1085-1092.

[11] Adamovich, Y., L. Rousso-Noori, Z. Zwighaft, A. Neufeld-Cohen, M. Golik, J. KrautCohen, et al., 2014. Circadian clocks and feeding time regulate the oscillations and levels of hepatic triglycerides. Cell Metab 19(2):319-330.

[12] Otway, D.T., S. Mantele, S. Bretschneider, J. Wright, P. Trayhurn, D.J. Skene, et al., 2011. Rhythmic diurnal gene expression in human adipose tissue from individuals who are lean, overweight, and type 2 diabetic. Diabetes 60(5):1577-1581.

[13] Wolff, G., M.J. Duncan, and K.A. Esser, 2013. Chronic phase advance alters circadian physiological rhythms and peripheral molecular clocks. J Appl Physiol (1985) 115(3):373-382.

[14] Peek, C.B., A.H. Affinati, K.M. Ramsey, H.Y. Kuo, W. Yu, L.A. Sena, et al., 2013. Circadian clock NAD+ cycle drives mitochondrial oxidative metabolism in mice. Science 342(6158):1243417.

[15] Feneberg, R. and B. Lemmer, 2004. Circadian rhythm of glucose uptake in cultures of skeletal muscle cells and adipocytes in Wistar-Kyoto, Wistar, Goto-Kakizaki, and spontaneously hypertensive rats. Chronobiol Int 21(4-5):521-538. 
[16] Zhang, R., N.F. Lahens, H.I. Ballance, M.E. Hughes, and J.B. Hogenesch, 2014. A circadian gene expression atlas in mammals: implications for biology and medicine. Proc Natl Acad Sci U S A 111(45):16219-16224.

[17] Reddy, A.B., N.A. Karp, E.S. Maywood, E.A. Sage, M. Deery, J.S. O'Neill, et al., 2006. Circadian orchestration of the hepatic proteome. Curr Biol 16(11):1107-1115.

[18] Robles, M.S., J. Cox, and M. Mann, 2014. In-vivo quantitative proteomics reveals a key contribution of post-transcriptional mechanisms to the circadian regulation of liver metabolism. PLoS Genet 10(1):e1004047.

[19] Pittendrigh, C.S., 1993. Temporal organization: reflections of a Darwinian clockwatcher. Annu Rev Physiol 55(16-54.

[20] Goodpaster, B.H. and L.M. Sparks, 2017. Metabolic Flexibility in Health and Disease. Cell Metab 25(5):1027-1036.

[21] Conroy, R.T. and M. O'Brien, 1974. Proceedings: Diurnal variation in athletic performance. J Physiol 236(1):51P.

[22] Facer-Childs, E. and R. Brandstaetter, 2015. Circadian Phenotype Composition is a Major Predictor of Diurnal Physical Performance in Teams. Front Neurol 6(208.

[23] Lamia, K.A., K.F. Storch, and C.J. Weitz, 2008. Physiological significance of a peripheral tissue circadian clock. Proc Natl Acad Sci U S A 105(39):15172-15177.

[24] Marcheva, B., K.M. Ramsey, E.D. Buhr, Y. Kobayashi, H. Su, C.H. Ko, et al., 2010. Disruption of the clock components CLOCK and BMAL1 leads to hypoinsulinaemia and diabetes. Nature 466(7306):627-631.

[25] Sadacca, L.A., K.A. Lamia, A.S. deLemos, B. Blum, and C.J. Weitz, 2011. An intrinsic circadian clock of the pancreas is required for normal insulin release and glucose homeostasis in mice. Diabetologia 54(1):120-124.

[26] Paschos, G.K., S. Ibrahim, W.L. Song, T. Kunieda, G. Grant, T.M. Reyes, et al., 2012. Obesity in mice with adipocyte-specific deletion of clock component Arntl. Nat Med 18(12):1768-1777.

[27] Shostak, A., J. Meyer-Kovac, and H. Oster, 2013. Circadian regulation of lipid mobilization in white adipose tissues. Diabetes 62(7):2195-2203.

[28] Dyar, K.A., S. Ciciliot, L.E. Wright, R.S. Bienso, G.M. Tagliazucchi, V.R. Patel, et al., 2014. Muscle insulin sensitivity and glucose metabolism are controlled by the intrinsic muscle clock. Mol Metab 3(1):29-41.

[29] Andrews, J.L., X. Zhang, J.J. McCarthy, E.L. McDearmon, T.A. Hornberger, B. Russell, et al., 2010. CLOCK and BMAL1 regulate MyoD and are necessary for maintenance of skeletal muscle phenotype and function. Proc Natl Acad Sci U S A 107(44):1909019095.

[30] Kumar Jha, P., E. Challet, and A. Kalsbeek, 2015. Circadian rhythms in glucose and lipid metabolism in nocturnal and diurnal mammals. Mol Cell Endocrinol 418 Pt 1(7488.

[31] Shi, S.Q., T.S. Ansari, O.P. McGuinness, D.H. Wasserman, and C.H. Johnson, 2013. Circadian disruption leads to insulin resistance and obesity. Curr Biol 23(5):372-381.

[32] Turek, F.W., C. Joshu, A. Kohsaka, E. Lin, G. Ivanova, E. McDearmon, et al., 2005. Obesity and metabolic syndrome in circadian Clock mutant mice. Science 308(5724):1043-1045.

[33] Lee, J., M.S. Kim, R. Li, V.Y. Liu, L. Fu, D.D. Moore, et al., 2011. Loss of Bmal1 leads to uncoupling and impaired glucose-stimulated insulin secretion in beta-cells. Islets $3(6)$. 
[34] Stephan, F.K. and I. Zucker, 1972. Circadian rhythms in drinking behavior and locomotor activity of rats are eliminated by hypothalamic lesions. Proc Natl Acad Sci U S A 69(6):1583-1586.

[35] Moore, R.Y. and V.B. Eichler, 1972. Loss of a circadian adrenal corticosterone rhythm following suprachiasmatic lesions in the rat. Brain Res 42(1):201-206.

[36] Ralph, M.R., R.G. Foster, F.C. Davis, and M. Menaker, 1990. Transplanted suprachiasmatic nucleus determines circadian period. Science 247(4945):975-978.

[37] Hara, R., K. Wan, H. Wakamatsu, R. Aida, T. Moriya, M. Akiyama, et al., 2001. Restricted feeding entrains liver clock without participation of the suprachiasmatic nucleus. Genes Cells 6(3):269-278.

[38] Wolff, G. and K.A. Esser, 2012. Scheduled exercise phase shifts the circadian clock in skeletal muscle. Med Sci Sports Exerc 44(9):1663-1670.

[39] Scheer, F.A., M.F. Hilton, C.S. Mantzoros, and S.A. Shea, 2009. Adverse metabolic and cardiovascular consequences of circadian misalignment. Proc Natl Acad Sci U S A 106(11):4453-4458.

[40] Morris, C.J., J.N. Yang, J.I. Garcia, S. Myers, I. Bozzi, W. Wang, et al., 2015. Endogenous circadian system and circadian misalignment impact glucose tolerance via separate mechanisms in humans. Proc Natl Acad Sci U S A 112(17):E2225-2234.

[41] Whichelow, M.J., R.A. Sturge, H. Keen, R.J. Jarrett, L. Stimmler, and S. Grainger, 1974. Diurnal variation in response to intravenous glucose. Br Med J 1(5906):488-491.

[42] Verrillo, A., A. De Teresa, C. Martino, G. Di Chiara, M. Pinto, L. Verrillo, et al., 1989. Differential roles of splanchnic and peripheral tissues in determining diurnal fluctuation of glucose tolerance. Am J Physiol 257(4 Pt 1):E459-465.

[43] Gibson, T. and R.J. Jarrett, 1972. Diurnal variation in insulin sensitivity. Lancet 2(7784):947-948.

[44] Biston, P., E. Van Cauter, G. Ofek, P. Linkowski, K.S. Polonsky, and J.P. Degaute, 1996. Diurnal variations in cardiovascular function and glucose regulation in normotensive humans. Hypertension 28(5):863-871.

[45] Van Cauter, E., E.T. Shapiro, H. Tillil, and K.S. Polonsky, 1992. Circadian modulation of glucose and insulin responses to meals: relationship to cortisol rhythm. Am J Physiol 262(4 Pt 1):E467-475.

[46] Versteeg, R.I., D.J. Stenvers, D. Visintainer, A. Linnenbank, M.W. Tanck, G. Zwanenburg, et al., 2017. Acute Effects of Morning Light on Plasma Glucose and Triglycerides in Healthy Men and Men with Type 2 Diabetes. J Biol Rhythms 32(2):130-142.

[47] Karatsoreos, I.N., S. Bhagat, E.B. Bloss, J.H. Morrison, and B.S. McEwen, 2011. Disruption of circadian clocks has ramifications for metabolism, brain, and behavior. Proc Natl Acad Sci U S A 108(4):1657-1662.

[48] Wehrens, S.M.T., S. Christou, C. Isherwood, B. Middleton, M.A. Gibbs, S.N. Archer, et al., 2017. Meal Timing Regulates the Human Circadian System. Curr Biol.

[49] Garaulet, M., P. Gomez-Abellan, J.J. Alburquerque-Bejar, Y.C. Lee, J.M. Ordovas, and F.A. Scheer, 2013. Timing of food intake predicts weight loss effectiveness. Int J Obes (Lond) 37(4):604-611.

[50] Archer, S.N., E.E. Laing, C.S. Moller-Levet, D.R. van der Veen, G. Bucca, A.S. Lazar, et al., 2014. Mistimed sleep disrupts circadian regulation of the human transcriptome. Proc Natl Acad Sci U S A 111(6):E682-691. 
[51] Merikanto, I., T. Lahti, H. Puolijoki, M. Vanhala, M. Peltonen, T. Laatikainen, et al., 2013. Associations of chronotype and sleep with cardiovascular diseases and type 2 diabetes. Chronobiol Int 30(4):470-477.

[52] Yu, J.H., C.H. Yun, J.H. Ahn, S. Suh, H.J. Cho, S.K. Lee, et al., 2015. Evening chronotype is associated with metabolic disorders and body composition in middle-aged adults. J Clin Endocrinol Metab 100(4):1494-1502.

[53] Garaulet, M., M.D. Corbalan, J.A. Madrid, E. Morales, J.C. Baraza, Y.C. Lee, et al., 2010. CLOCK gene is implicated in weight reduction in obese patients participating in a dietary programme based on the Mediterranean diet. Int J Obes (Lond) 34(3):516523.

[54] Kelly, M.A., S.D. Rees, M.Z. Hydrie, A.S. Shera, S. Bellary, J.P. O'Hare, et al., 2012. Circadian gene variants and susceptibility to type 2 diabetes: a pilot study. PLoS One 7(4):e32670.

[55] Mu, C., Y. Yang, and W. Zhu, 2016. Gut Microbiota: The Brain Peacekeeper. Front Microbiol 7(345.

[56] Thaiss, C.A., D. Zeevi, M. Levy, G. Zilberman-Schapira, J. Suez, A.C. Tengeler, et al., 2014. Transkingdom control of microbiota diurnal oscillations promotes metabolic homeostasis. Cell 159(3):514-529.

[57] Leone, V., S.M. Gibbons, K. Martinez, A.L. Hutchison, E.Y. Huang, C.M. Cham, et al., 2015. Effects of diurnal variation of gut microbes and high-fat feeding on host circadian clock function and metabolism. Cell Host Microbe 17(5):681-689.

[58] Stenvers, D.j., Light, the circadian timing system, and type 2 diabetes, in The diurnal rhythm of adipose tissue gene expression is reduced in obese patients with type 2 diabetes. 2017, AMC: Amsterdam. p. 109-146.

[59] Canto, C. and J. Auwerx, 2009. PGC-1alpha, SIRT1 and AMPK, an energy sensing network that controls energy expenditure. Curr Opin Lipidol 20(2):98-105. 


\title{
Appendix
}

\author{
Summary \\ Zusammenfassung \\ Valorization \\ Acknowledgements \\ List of publications
}

About the Author 


\section{Summary}

The growing increase in the prevalence of obesity and type 2 diabetes mellitus (T2D) imposes an important global health issue, as this is associated with high co-morbidity and mortality and causes a major burden on health care costs. Indeed, the major determinants of obesity and T2D are high caloric intake and reduced physical activity, but these factors cannot entirely explain the increasing prevalence of these clinical manifestations. Hence, it has been speculated that this development could be attributed to drastic lifestyle changes in response to the industrialization of our modern society, which may not be restricted to changes in diet and physical activity [1]. More particular, disruption of the biological rhythm has been subjected to play a central role in metabolic disease development. Such disruption can occur when our internal biological clock is misaligned to our behavioral cycle (food, sleep, activity), as in across time-zone travel. Misalignment can also occur in more common situations such as working shifts, partying through the night or sleeping in on weekends also known as 'social jetlag'. In Central Europe over $70 \%$ of a surveyed population suffers from social jetlag [2]. To date, it is not fully understood how disturbances of the biological clock promote the risk to develop obesity and T2D; yet epidemiological studies demonstrate compelling evidence for an association of shift work [3], late meal timing [4], late chronotype [5] and social jetlag [6] with increased risk for obesity and T2D. Hence, in this thesis we examined the possible link between the disruption of the human biological clock and derangements in human energy metabolism, and investigated to which extent this contributes to the etiology of obesity and type 2 diabetes mellitus.

In type 2 diabetes etiology altered skeletal muscle mitochondrial metabolism and insulin resistance are central, therefore we employed two strategies to examine the role of the skeletal muscle molecular clock: First, a cellular model was set up and used (CHAPTER 2, 3, 4) to characterize the molecular clock in skeletal muscle of healthy, obese, and type 2 diabetes patient donors. Subsequent human intervention studies were conducted to characterize the human energy metabolism under normal and misalignment conditions (CHAPTER 5, 6). 
A methodological approach is described in CHAPTER 2 that lead to the development of a skeletal muscle cellular model to investigate circadian rhythms in vitro. For this purpose, we discuss the use of straight versus parallel timeline synchronization. In line with this, data reliability for time point and time course robustness was demonstrated by introduction of multiple reference gene correction, rather than conventional single gene correction. Serum shock was used to align the cellular rhythms of the individual cells in culture. Synchronization was highly reproducible also between cultures of different donors. Analysis of clock and metabolic genes at baseline was used to confirm that serum shock did not adversely alter target gene expression nor donor metabolic phenotype in culture. Intriguingly, we encountered first indications of altered gene expression between cultures of sedentary lean healthy subjects and type 2 diabetes patients for clock genes and regulators of the molecular clock (CLOCK, REVERBA and SIRT1).

Subsequently, in CHAPTER 3 this model was used to investigate whether circadian rhythms were different amongst donors with different states of metabolic health. Hence, we examined circadian rhythms in in vitro synchronized cultures of donors with a wide range in metabolic health; from athletes to lean sedentary men, and obese but otherwise healthy men to obese type 2 diabetes patients. Over a 72-hour time course synchronized cultures of all four groups displayed robust circadian rhythms in core clock gene expression. Additionally, we found modest rhythmic expression of key-regulators in carbohydrate and lipid metabolism. It is noteworthy, that regulatory genes of the molecular clock with input from metabolic pathways, such as REVERBA and SIRT1 were dampened in amplitude in cultures of type 2 diabetes patients. Intriguingly, both genes are important regulators in energy metabolism and also in mitochondrial biogenesis.

Hence, in CHAPTER 4, we proceeded with the cellular model to explore whether such differences translate to changes in mitochondrial respiration, and to answer whether human mitochondrial function is intrinsically rhythmic. However, observed changes in mitochondrial respiration in a time course of 30 hours with 6-hourly measurements did not resemble rhythmicity. Unexpectedly, we encountered pronounced rhythmicity in protein content in cultures of all groups; the reason for the 24-hour variation in total cellular protein content could not be revealed from the present study. Yet, regardless of total protein 
content, a markedly reduced mitochondrial function could be observed in cultures of type 2 diabetes donors at all time points, in comparison to the groups.

With the novel findings from the preceding chapters, in CHAPTER 5 we hypothesized that human skeletal muscle is also rhythmic in vivo, as well as whole-body energy metabolism. To examine this, in CHAPTER 5 young, lean, healthy men were enrolled in a standardized protocol with regular meals, physical activity and sleep to reflect our everyday lifestyle. Skeletal muscle biopsies were taken 5-hourly for 24 hours to assess mitochondrial function and gene expression. In addition, whole-body resting energy expenditure and substrate oxidation were measured. Indeed, clock expression was found to be circadian in human skeletal muscle. Interestingly, also skeletal muscle mitochondrial function, assessed by ex vivo high resolution respirometry, revealed a 24-hour rhythm, with a peak at 11PM and a trough at 1PM. Intriguingly, the peak of whole-body energy expenditure coincided with highest skeletal mitochondrial function, while being lowest at 4AM. These findings are of relevance, as type 2 diabetes is characterized by low energy expenditure and reduced mitochondrial function. It is tempting to speculate that disturbing the day-night rhythm could derange metabolic health by altering mitochondrial function.

It has been indicated by animal studies that the molecular clock drives important tissuespecific functions, that orchestrate whole-body homeostasis. Given the findings of the preceding chapter, we hypothesized that the molecular clock under circadian misalignment would not re-align rapidly resulting in metabolic disturbances such as insulin resistance and altered mitochondrial function. To this end, as described in CHAPTER 6, we conducted a randomized cross-over trial at which healthy young male subjects were examined during a 3-day control period and a 3.5-day misalignment period (12-hour rapid shift). Metabolism was assessed by measuring insulin sensitivity, mitochondrial function and whole-body energy expenditure. Indeed, under circadian misalignment the skeletal muscle molecular clock did not re-align to the new behavioral rhythm within 3 days. Furthermore, circadian misalignment led to decreased peripheral insulin sensitivity and disturbed plasma metabolites. Ultimately, increased mitochondrial oxidative capacity as well as increased sleeping metabolic rate, indicated mistimed and impaired mitochondrial efficiency. 
Together, these findings support the association of circadian misalignment with the increased risk to develop type 2 diabetes.

As discussed in CHAPTER 7, these findings underscore that circadian misalignment as in Jetlag-conditions can inflict metabolic disturbances in healthy subjects. Hence, disruption of the biological clock by altered gene expression or circadian misalignment could indeed contribute to the development of metabolic disease such as type 2 diabetes mellitus. 


\section{Zusammenfassung}

Die stetige Zunahme von Obesitas und Typ 2 Diabetes Mellitus (T2D) stellt ein großes gesundheitliches Problem dar, da diese mit Folgeerkrankungen und einer erhöhten Sterblichkeitsrate einhergeht. Darüber hinaus verursachen diese Erkrankungen weltweit eine hohe finanzielle Belastung für das Gesundheitssystem. Es ist bekannt, dass zu den Risikofaktoren von Obesitas und Typ 2 Diabetes sowohl eine hochkalorische Ernährung als auch eine geringe körperliche Aktivität gehören, doch diese Faktoren alleine können die zunehmende Prävalenz dieser Krankheitsbilder nicht vollständig erklären. Deshalb wird schon seit längerem vermutet, dass diese Entwicklung mit den drastischen LebensstilVeränderungen der heutigen Gesellschaft zusammenhängt, welche sich aus der Industrialisierung ergeben und sich nicht nur auf Veränderungen in der Ernährung und der körperlichen Aktivität beschränken. Es wird davon ausgegangen, dass eine Störung der biologischen Uhr eine maßgebliche Rolle bei der Entwicklung von Stoffwechselerkrankungen spielt. Diese Störungen kommen zustande, wenn unsere innere biologische Uhr nicht synchron zu unserem Verhalten läuft. Damit ist gemeint, dass die Nahrungsaufnahme, der Schlaf und die Aktivitätsphasen asynchron zu unserer inneren Uhr laufen, wie es z.B. bei Interkontinentalflügen der Fall ist. Doch dieser Zustand entsteht noch viel häufiger in unserem alltäglichen Leben, nämlich immer dann, wenn z.B. Schichtarbeit, „Durchfeiern“, oder langes Ausschlafen am Wochenende praktiziert werden. Dieser Zustand wird als „soziales Jetlag“ bezeichnet. Nach Erhebungen in Zentraleuropa sind mittlerweile mehr als 70\% der Bevölkerung davon betroffen [3]. Zurzeit ist noch nicht geklärt, wie die Störungen der biologischen Uhr das Risiko fettleibig zu werden und Typ 2 Diabetes zu entwickeln, begünstigen. Jedoch weisen epidemiologische Studien darauf hin, dass Schichtarbeit [4], späte Nahrungsaufnahme am Abend [5], später Chronotyp [6] und „soziales Jetlag“ [7] das Risiko erhöhen Obesitas und Typ 2 Diabetes zu entwickeln.

Vor diesem Hintergrund wurde in der vorliegenden Studie genau diese mögliche Verbindung zwischen der biologischen Uhr und dem humanen Stoffwechsel untersucht, mit dem Ziel mögliche Mechanismen in der Ätiologie von Obesitas und Typ 2 Diabetes zu identifizieren.

Charakteristisch für Typ 2 Diabetes ist die in Skelettmuskulatur vorliegende verminderte mitochondriale Kapazität sowie eine Insulinresistenz. Daher wurden zwei Strategien 
angewendet, um die Rolle der molekularen biologischen Uhr im Skelettmuskel zu untersuchen. Zuerst wurde ein zelluläres Modell entwickelt und genutzt (Kapitel 2, 3, 4), um die molekulare Uhr des Skelettmuskels von gesunden, fettsüchtigen und Typ 2 Diabetikern zu charakterisieren. Des Weiteren wurde der Stoffwechsel in Humanstudien unter normalen Lebensbedingungen sowie nach einer zirkadianen Phasenverschiebung charakterisiert (Kapitel 5, 6).

Die methodische Entwicklung des Zellmodells zur Untersuchung, ob zirkadiane Rhythmen intrinsisch in Skeletettmuskelzellen erzeugt werden können, wird in Kapitel 2 beschrieben. Zu diesem Zweck wird zuerst der Nutzen eines singulären sowie parallelen ZeitlinienAnsatzes demonstriert. Des Weiteren werden die Referenzgenpool-Normalisierung der Normalisierung mit einzelnen Referenzgenen gegenübergestellt, um die gesteigerte Robustheit der Normalisierung für einzelne Datenpunkte sowie Daten im Experimentenverlauf zu demonstrieren. Mit dem Ziel die individuellen Zellen einer Zellkultur zur Synchronisation anzuregen, wurden diese kurzzeitig mit Serum behandelt. Die Synchronisation war bei allen Spenderkulturen reproduzierbar. Eine Quantifizierung der molekularen Uhr-Gene sowie weiterer Gene des Stoffwechsels zeigten, dass die Prozedur der Synchronisation nicht den Phänotyp des Spenderstoffwechsels in den Zellkulturen beeinträchtigte.

Interessanterweise konnten bei dieser ersten Analyse Unterschiede in der Expression von Genen der molekularen Uhr sowie deren Regulatoren in den Zellkulturen von gesunden und Typ 2 Diabetikern festgestellt werden.

Anschließend wurde dieses Zellmodel in Kapitel 3 zur Anwendung gebracht, um zu untersuchen, ob die zirkadiane Rhythmik durch den Spenderstoffwechsel in Zellkulturen verändert ist. Hierfür wurden Spenderzellen von Ausdauersportlern mit denen von schlanken gesunden Nichtsportlern verglichen. Des Weiteren wurden Spenderzellen von fettsüchtigen, aber darüber hinaus gesunden Freiwilligen, mit denen von Typ 2 Diabetikern verglichen. Über einen erfassten Zeitverlauf von 72 Stunden zeigten die synchronisierten Kulturen aller vier Gruppen robuste zirkadiane Rhythmen in der Genexpression der molekularen Uhr. Ferner wurden nur moderate Rhythmen in Stoffwechselgenen des Kohlehydrat- und Fettstoffwechsels festgestellt. Auffallend war jedoch, dass regulatorische 
Gene der molekularen Uhr, die als Sensoren des Energiestoffwechsels wirken, (REVERBA und SIRT1) einen gedämpften Rhythmus in Zellkulturen der Typ 2 Diabetiker aufzeigten. Es handelt sich bei diesen Genen überdies um zentrale Regulatoren des Energiestoffwechsels und der mitochondrialen Biogenese.

Um zu untersuchen, ob diese Unterschiede in der Genexpression zu einer Veränderung im mitochondrialen Stoffwechsel führen könnten, und auch um zu beantworten, ob mitochondriale Kapazität einen intrinsischen zirkadianen Rhythmus hat, wurde erneut das Zellmodel verwendet, wie in Kapitel 4 beschrieben. Hierzu wurde die mitochondriale Kapazität in einem Zeitverlauf von 30 Stunden 6-stündlich gemessen. Unerwarteterweise wurde nicht im mitochondrialen Stoffwechsel ein Rhythmus festgestellt, sondern in dem Gesamtproteingehalt der gemessenen Proben. Die Ursache für diese in allen Gruppen festgestellte rhythmische Fluktuation konnte in der vorliegenden Studie nicht abschließend geklärt werden. Jedoch, unabhängig vom Proteingehalt, konnte eine starke Reduktion der mitochondrialen Kapazität zu allen Zeitpunkten in den Zellkulturen der Typ 2 Diabetiker festgestellt werden.

Mit den Erkenntnissen der vorangehenden Kapitel wurde in Kapitel $\mathbf{5}$ die Hypothese aufgestellt, dass der Energiestoffwechsel sowohl auf Ebene des Skelettmuskels als auch im gesamten Körper rhythmisch ist. Um dies zu untersuchen, wurden schlanke, junge männliche Freiwillige unter standardisierten normalen Lebensbedingungen gemessen (normale Mahlzeiten, durchschnittliche körperliche Aktivität und normaler Schlaf). Im Verlauf von 24 Stunden wurden 5-stündlich die mitochondriale Kapazität, der Ganzköperstoffwechsel und die Genexpression gemessen.

Damit konnte bestätigt werden, dass in der Tat auch im Skelettmuskelgewebe die Expression von molekularen Uhr-Genen zirkadianer Natur ist. Auffallenderweise zeigten sowohl die mitochondriale Kapazität des Skelettmuskels als auch der Ganzkörperstoffwechsel einen Tag-Nacht-Rhythmus, der sein Maximum um 23 Uhr erreichte. Hingegen lag der Tiefpunkt des Ganzkörperstoffwechsels um 4 Uhr nachts vor, während dieser erst um 13 Uhr bei der mitochondrialen Kapazität des Skelettmuskels auftrat. 
Mit dem Vorwissen, dass Typ 2 Diabetes durch eine niedrigere mitochondriale und Ganzkörperstoffwechselaktivität charakterisiert ist, liegt die Vermutung nahe, dass eine Störung des Tag-Nacht-Rhythmus den Ganzkörperstoffwechsel durch eine veränderte mitochondriale Kapazität nachteilig beeinflussen kann.

Erkenntnisse aus Tierversuchen haben bereits Hinweise darauf geliefert, dass die molekulare Uhr wichtige gewebespezifische Funktionen steuert, um das Gleichgewicht des Körpers zu begünstigen. Anhand der vorangegangenen Studien wurde in Kapitel 6 die Hypothese aufgestellt, dass sich bei einer akuten Verschiebung der zirkadianen Phase die molekulare Uhr nicht an die neue Phase anpasst, wodurch Skelettmuskelfunktionen gestört werden können, wie z.B. die mitochondriale Kapazität und die Insulinsensitivität.

Um dies näher zu untersuchen, wurden junge gesunde männliche Freiwillige für 3 Tage unter normalen Lebensbedingungen und für 3,5 Tage zur zirkadianen Phasenverschiebung einem standardisierten Alltag unterzogen. Die Analyse der Genexpression zeigte, dass die Gene der molekularen Uhr tatsächlich den ursprünglichen Rhythmus beibehalten. Stoffwechselbasierte Messungen der Probanden ergaben, dass bei Phasenverschiebung eine Reduktion der Insulinsensitivität auftritt. Des Weiteren konnte eine erhöhte mitochondriale Kapazität im Skelettmuskel sowie ein im Schlaf erhöhter Energiestoffwechsel vorgefunden werden; letztere Ergebnisse weisen auf eine verminderte mitochondriale Effizienz hin.

Wie in Kapitel 7 ausführlich diskutiert, unterstreichen diese Erkenntnisse die Vermutung, dass zirkadiane Phasenverschiebung, wie sie im "sozialen Jetlag“ vorkommt, Stoffwechselstörungen in gesunden Menschen hervorrufen kann. Dementsprechend könnte die Störung der biologischen Uhr, durch sowohl veränderte Genexpression als auch durch zirkadiane Phasenverschiebung, zu der Entstehung von Stoffwechselstörungen wie Typ 2 Diabetes beitragen. 


\section{Valorization}

\section{Background}

Through the last century, changes in social- and economic status together with technological advance such as artificial light and fridges have enabled humans to disengage from their primordial day-night rhythm. Obviously, this comes not without risks. Since 1980 the obesity prevalence has more than doubled in over 70 countries [7], while the number of T2D diagnoses almost quadrupled [8]. This development entails increased risks for obesity related comorbidities, ultimately resulting in shortened burden free lifetime and overall life expectancy [7, 9]. Further, epidemiological studies indicate that the recent lifestyle changes contribute to a disruption of day-night rhythm, which has been associated with negative consequences for metabolic health $[3,6,10,11]$. With this in mind, it is important to realize that about $15-20 \%$ of the working population is involved in shift work or regularly travels across several time zones [12]. However, disengaging from our natural day-night rhythm is not limited to occupational environment, but rather has become an indispensable part of our society. In fact, in Central Europe over $70 \%$ of a surveyed population suffers from social jetlag [2] - a condition at which one suffers from a jetlag imposed by social aspects, such as working in shift, partying through the night or sleeping in on weekends. Similar to travelling time-zones, social jetlag occurs by a misalignment of the behavioral cycle (food, sleep, activity) with the internal biological clock. To date, it is not fully understood how disturbances of the biological clock promote the risk to develop obesity and T2D.

\section{Target groups and clinical relevance}

The manifestation of obesity and type 2 diabetes is mainly caused by behavioral and environmental factors, such as low level of physical activity and energy dense diets. It has been shown that potent and cost-effective treatment strategies can be increasing physical activity and reducing calorie intake to improve skeletal muscle mitochondrial function, peripheral insulin sensitivity and overall metabolic health. Yet, such interventions are difficult to implement and maintain in a population that has been living the opposite lifestyle for years. In the light of the central aspect of social jetlag, it appears to be even more difficult to expect sufficient compliance. The difficulties are versatile e.g. rapidly 
changing schedules can impede implementing new habits, food consumption at arbitrary times can alter social interaction and choice for food, tiredness can impact willingness and capability to comply with food and exercise regimes. Nevertheless, epidemiological studies demonstrate compelling evidence for an association of shift work [3], late meal timing [4], late chronotype [5] and social jetlag [6] with increased risk for obesity and T2D, thus demanding effective treatment strategies.

Additionally, as reported in this thesis, we included healthy young participants enduring a 12-hour circadian misalignment. In contrast to the target population, these participants were guided to maintain a normal lifestyle with respect to diet, activity and sleep, also during misalignment. Yet, without altering food intake, physical activity or sleep duration, circadian misalignment in healthy young participants led to acute decreased insulin sensitivity and adverse energy metabolism. It should be noted, that dietary choices and physical activity in individuals in real life may alter vastly due to aspects of free choice and also environmental availability (e.g. healthy food prepared freshly at 3 AM) [13-15]. Thereby, the potential metabolic health threat for the population suffering regularly from jetlag may likely be even larger than demonstrated in our healthy volunteers.

\section{Innovation}

In this thesis, we used a two-strategy approach employing in vitro and in vivo studies to investigate the link between biological clock disruption and deranged energy metabolism. We first targeted to prove that human skeletal muscle cells indeed employ a molecular clockwork and to further examine whether such clockwork might be altered by metabolic state. To this end, we raised a unique cell bank of primary skeletal muscle cells of human donors ranging in metabolic health. This cell bank we used to compare intrinsic (genetic and possibly epigenetic) aspects of the skeletal muscle molecular clock and metabolism isolated from donor (patho)physiology. In this thesis, as indicated by the in vitro model studies, rhythmic expression of metabolic regulators of the molecular clock are dampened in wellsynchronized cells of type 2 diabetes patients. Thus, we identified two regulatory intersections that could serve as promising targets in novel drug prevention and treatment strategies. Further, the general concept of this model may also be used for fine-tuning treatment timing to improve drug efficacy of novel and existing drugs. 
Furthermore, with the state of the art Metabolic Research Unit Maastricht, we were capable of providing authentic 'normal - but standardized - living conditions' to examine biological rhythms in human energy metabolism. Most comparable human studies so far were using complex behavioral intervention routines with limited translation to normal living conditions. With the human studies performed in this thesis, detailed characterization of skeletal muscle and whole body metabolism under 'normal day' conditions were achieved. Especially, showing that acute 12-hour circadian misalignment itself can lead to substantial metabolic adverse events, such as decreased peripheral insulin sensitivity and altered mitochondrial function in healthy young lean volunteers is an alarming result. It is tempting to speculate, that individuals with severe overweight or type 2 diabetes may suffer from greater health implications upon circadian misalignment, due to manifested reduced metabolic flexibility.

\section{Societal relevance}

Over the last decades, awareness about circadian rhythm has been raising interest in both academia and industry. Current technological advances have made it fairly easy to monitor behavioral and physiological aspects of our everyday lives. Mobile phones and wearables nowadays can measure by default e.g. light exposure, heart rate, physical activity levels, and can be used to actively assist in implementing lifestyle changes. Although, these applications and devices do not always reach the standards necessary to be useful in assisting to implement a healthy lifestyle, but a trend towards improved usability and functionality is undeniable. However, the current hype that increased the availability of these 'technological advances' to the general population must be noticed with care, as the predictive value for individuals with regard to their health advantages can be limited.

Furthermore, the findings from this thesis underscore the idea that treatment strategies for metabolic diseases such as type 2 diabetes may benefit from implementing chronotherapeutic approaches. This could be realized on different levels, such as modifying artificial light and ambient temperature in housing and nursing environment, but also timed administration of drugs that were fine-tuned to the time of highest efficacy. Additionally, given the technological advances, personalized day-night rhythm coaching, considering 
aspects of social and work life, healthy diet and physical activity level may be implemented to strengthen current prevention and treatment strategies.

\section{Conclusion}

This thesis has shed light on the subjected link between disturbances of the molecular clock and human metabolic health. A vast majority of the world's population is at risk for a disturbed biological clock and therefore is at risk to suffer from metabolic derangement. Hence, profound understanding by which the disruption of the biological clock leads to metabolic disease is needed to provide effective treatment strategies. Here, we identified two molecular intersections between the molecular clock and metabolism that are altered in type 2 diabetes. Further, we were able to demonstrate that maximal circadian misalignment leads to serious metabolic derangements in young healthy men. This alarming result is important, as the vast majority of individuals regularly exposed to social jetlag is of lower metabolic health, and hence may be at even greater risk to develop adverse metabolic conditions. 


\section{References}

[1] Qian, J. and F.A. Scheer, 2016. Circadian System and Glucose Metabolism: Implications for Physiology and Disease. Trends Endocrinol Metab 27(5):282-293.

[2] Roenneberg, T., K.V. Allebrandt, M. Merrow, and C. Vetter, 2012. Social jetlag and obesity. Curr Biol 22(10):939-943.

[3] Pan, A., E.S. Schernhammer, Q. Sun, and F.B. Hu, 2011. Rotating night shift work and risk of type 2 diabetes: two prospective cohort studies in women. PLoS Med 8(12):e1001141.

[4] Garaulet, M., P. Gomez-Abellan, J.J. Alburquerque-Bejar, Y.C. Lee, J.M. Ordovas, and F.A. Scheer, 2013. Timing of food intake predicts weight loss effectiveness. Int J Obes (Lond) 37(4):604-611.

[5] Yu, J.H., C.H. Yun, J.H. Ahn, S. Suh, H.J. Cho, S.K. Lee, et al., 2015. Evening chronotype is associated with metabolic disorders and body composition in middle-aged adults. J Clin Endocrinol Metab 100(4):1494-1502.

[6] Parsons, M.J., T.E. Moffitt, A.M. Gregory, S. Goldman-Mellor, P.M. Nolan, R. Poulton, et al., 2015. Social jetlag, obesity and metabolic disorder: investigation in a cohort study. Int J Obes (Lond) 39(5):842-848.

[7] Collaborators, G.B.D.O., 2017. Health Effects of Overweight and Obesity in 195 Countries over 25 Years. N Engl J Med.

[8] Collaboration, N.C.D.R.F., 2016. Worldwide trends in diabetes since 1980: a pooled analysis of 751 population-based studies with 4.4 million participants. Lancet 387(10027):1513-1530.

[9] Global Burden of Metabolic Risk Factors for Chronic Diseases, C., 2014. Cardiovascular disease, chronic kidney disease, and diabetes mortality burden of cardiometabolic risk factors from 1980 to 2010: a comparative risk assessment. Lancet Diabetes Endocrinol 2(8):634-647.

[10] Suwazono, Y., M. Dochi, M. Oishi, K. Tanaka, E. Kobayashi, and K. Sakata, 2009. Shiftwork and impaired glucose metabolism: a 14-year cohort study on 7104 male workers. Chronobiol Int 26(5):926-941.

[11] Mattson, M.P., D.B. Allison, L. Fontana, M. Harvie, V.D. Longo, W.J. Malaisse, et al., 2014. Meal frequency and timing in health and disease. Proc Natl Acad Sci U S A 111(47):16647-16653.

[12] Haus, E. and M. Smolensky, 2006. Biological clocks and shift work: circadian dysregulation and potential long-term effects. Cancer Causes Control 17(4):489-500.

[13] Lennernas, M., L. Hambraeus, and T. Akerstedt, 1995. Shift related dietary intake in day and shift workers. Appetite 25(3):253-265.

[14] Bonnell, E.K., C.E. Huggins, C.T. Huggins, T.A. McCaffrey, C. Palermo, and M.P. Bonham, 2017. Influences on Dietary Choices during Day versus Night Shift in Shift Workers: A Mixed Methods Study. Nutrients 9(3).

[15] Bonham, M.P., E.K. Bonnell, and C.E. Huggins, 2016. Energy intake of shift workers compared to fixed day workers: A systematic review and meta-analysis. Chronobiol Int 33(8):1086-1100. 


\section{Acknowledgements}

Well, now that the book is finished, it is time to thank those who supported and shaped this work and of course everyone who made this a pleasant and exciting journey!

At first, I would like to thank my promotor-team Patrick and Matthijs. It was a great path to walk along with you so far. I am truly grateful that I could learn so much from your different ways. You allowed me to be creative and guided me whenever possible. Patrick, I am not sure whether I know anyone who is that efficient as you are, but it was fascinating all along. Most certainly many drafts and final versions grew from these fast correction sessions, that in hindsight were more of a whirl than distinct Version 1, 2a, 3.4...

Matthijs, luckily you were there, too. Putting a hold on this fast pace, and taking the necessary time to have a look around. Always capable of revealing a possible outsider view, and if necessary a second or even a third way to interpret it.

To me it was encouraging and inspiring to see how your experience changed perspective and improved outcome. In these years, I valued (and cursed) most that you took the patience to leave it up to me, and guide me until I got it the right way.

Further I would like to thank Wouter and Joris, who guided me in my Junior Practical Internship, where I learnt the ins and outs about mitochondrial metabolism. Also, at this point my thanks to Anouk, who demanded that I talk Dutch to the subjects, although it was really really rudimental; I hope it improved a little since then (:)

At least I wasn't stopped by Silvie. Many thanks to you, too. In the Senior Practical Internship, on your side I learnt so many new methods and useful applications, that I cannot list it here. Especially, at the time when I applied for the NUTRIM NWO Graduate Programme Proposal, you were first in line to guide me by asking the right questions, so that I could improve, adapt and evolve the concept of this thesis project. Of course, at this point I would like to cordially thank NWO and NUTRIM for the trust and means that were invested in me and this project. To reach this point, I am grateful to have had the support from Rotary International, and the Rotary Club Leverkusen-Opladen in particular, who financially supported my initial master studies in Maastricht. 
My special thanks go to you Bart and Helene. Your expertise in circadian rhythms really propelled the progress of this thesis project, so that I could start from the very first minute. Our meetings in Maastricht and Lille were always extremely productive and paving the way for a new great idea. Bart, I will not forget the frank honesty and clarity of our creative brainstorm sessions.

Frank, from you I learnt a whole new perspective on circadian rhythms. Chronobiology is such a complex field, especially when considered on a physiological level, and I still learn a lot about it every day. I thank you for all your feedback and guidance.

Marlies, together with Silvie we had a nice kickstart, working hard together to get this beneficial nutritional compound tested. It was always nice, to have some recreational $M n^{\prime}$ Ming afterwards... Thinking back on that: You know it's all an illousiooon.

Dr. Dirk, my maaaan! Well I am not sure how we survived these nights. Certainly, our classic "Patrick trakteerd" breaks worked some miracles. Still, I am glad we did not listen to the hundreds of colleagues, who thought it would be a smart idea to measure ourselves alongside. Honestly, it's a pity for science that you dedicate your time to being a medical doctor $(;)$, hopefully you consider to re-enter academia soon. Together with Jakob we could achieve anything! But seriously, Jakob, how do you do that. Yes, yes, German genes, I know. But you are so good in planning. It was frightening and relieving to see how fast paced we could pull of this last and at the same time most extensive human study. It was great working with you guys. Most memorable was 5.30AM our get together after a nice unhealthy shift. Feierabend!!!

Niels. Best fun ever. Luckily you were there at some nights!

Marije, Vera, Sabine, Anne, Hannah, Nadja, Yvonne, Marlies and Lotte, we all know how terrible experiments and teaching are to our agendas, but together we somehow always managed to keep up the Maastricht spirit. 
Hannah, I am already missing the times of stop-and-go traffic, may it be in the bus, or at the building site of the A2 tunnel, that was finally finished to the end of our promotion trajectory. It was always fun and somehow, we magically managed to be done with our work at the same time $: ;$

Also, of course I would like to thank for the determined assistance in our lab. Gert, you took the time to teach me how to grow human primary cultures, one of the pillars in my thesis. Another pillar was high resolution respirometry, and Johanna, I am thankful for all the time you took to teach it to me. Esther, to me it feels like you were undoubtedly involved in almost every project, pilot, test or experiment. I am really grateful for your fast and accurate support and the open communication.

And to the rest of the SHOCk-Group I would like to thank you for supportive and collaborative time we had. It is all a great team effort that made these high quality studies possible. Keep up the spirit!

It cannot be stressed enough: It is you, the volunteers that participated in our (and of course other) studies, that we have to thank most. Many of you were driven by the ideal to further the scientific knowledge to serve a better progress in medicine for humans like you and everyone else. With the physically and also rather demanding study protocols that you chose to comply, I have to say I admire your spirit and am grateful that you were a part of this.

It is often the little things with which we can help others most! I want to express my gratitude to everyone, doing the little good deed to a colleague or friend, that help us out big time. An dieser Stelle Isa, danke für Deine prompte Hilfe!

Auch Dir Yogi, und Euch Jürgen und Prof. Wilting möchte ich danken für das Vertrauen und die Unterstützung, die ich auf meinem frühen Weg durch Euch erhalten habe.

Meiner Familie, Großeltern und Schwiegereltern und Freunden möchte ich danken. Ihr habt meistens Verständnis für die eigenartigen Experimente gehabt, und geholfen, wo Ihr 
konntet. An dieser Stelle möchte ich auch Anna-Maria Pastoors und dir Nici dafür danken, Hannah-Lynn in den sehr turbulenten Zeiten immer ein sicherer Hafen gewesen zu sein. Auch Dir Jan will ich danken, auch wenn wir nicht mehr so oft wie früher beisammen kommen, es war und ist immer wieder eine gute Zeit! Auf hoffentlich bald wieder eine Partie Squash, und den Marathon in 2018?

Mama und Papa, es war gewiss nicht immer einfach mich auf meinem Lebensweg zu begleiten und zu stärken. Dass ich diese Dissertation nun verteidigen darf, habe ich Eurer Ausdauer und Eurer Liebe zu verdanken. Danke, dass Ihr nie lockergelassen habt!

Von Herzen geht mein größter Dank an Dich Simone. Nicht nur für die unzähligen Nächte, die wir Seite an Seite durchgemacht haben, sondern auch, dass Du uns in dieser turbulenten Zeit unseren größten Wunsch, Eltern zu werden, schon zweimal erfüllt hast. Auch dafür, dass Du mit Liebe und Hingabe die Zeit ausgeglichen hast, in der Papa mal wieder wegen eines Experiments eine halbe Woche nicht da sein konnte. Danke, dass du immer für mich da bist!

Hannah-Lynn und Frederick, ich danke Euch für all die schönen Momente, die mich in dieser Zeit begleitet haben und den Blick für das wirklich Wichtige im Leben geschärft haben. 


\section{List of Publications}

[1] Hansen J, Timmers S, Moonen-Kornips E, Duez H,Staels B, Hesselink, MKC, Schrauwen P (2016) Synchronized human skeletal myotubes of lean, obese and type 2 diabetic patients maintain circadian oscillation of clock genes. Sci Rep 6: 35047

[2] van Moorsel D $=$, Hansen J , Havekes B, Scheer FA, Jorgensen JA, Hoeks J, Schrauwen-Hinderling VB, Duez H, Lefebvre P, Schaper NC, Hesselink, MKC, Staels B, Schrauwen P (2016) Demonstration of a day-night rhythm in human skeletal muscle oxidative capacity. Mol Metab 5: 635-645 (=, contributed equally)

[3] Timmers S, de Ligt M, Phielix E, van de Weijer T, Hansen J, Moonen-Kornips E, Schaart G, Kunz I, Hesselink MKC, Schrauwen-Hinderling VB, Schrauwen P (2016) Resveratrol as Add-on Therapy in Subjects With Well-Controlled Type 2 Diabetes: A Randomized Controlled Trial. Diabetes Care

[4] van der Lans AA, Hoeks J, Brans B, Vijgen GH,Visser MG,Vosselman MJ, Hansen J, Jorgensen JA, Wu J, Mottaghy FM, Schrauwen P, van Marken Lichtenbelt WD (2013) Cold acclimation recruits human brown fat and increases nonshivering thermogenesis. J Clin Invest 123: 3395-3403

[5] Becker J, Frohlich J, Hansen J, Zelent C, Perske C, Wilting J (2012) The lymphangiogenesis inhibitor esVEGFR-2 in human embryos: expression in sympatho-adrenal tissues and differentiation-induced up-regulation in neuroblastoma. Histol Histopathol 27: 721-733 


\section{About the author}

Jan was born in Düsseldorf Germany on January 7th 1986. Before his bachelor studies, he worked as trained Technical Assistant at the Molecular Hematology Institute, Frankfurt University. One year later, he was selected to follow the "Excellence Bachelor Program: Molecular Medicine" at Göttingen University. In parallel to his study program he was occupationally involved in several research projects at the Göttingen Centrum for Molecular Biosciences and the University Medical Center. For his extracurricular efforts in the student body and personal engagement, Jan was honored with the Rotary Ambassadorial Scholarship in order to finance his studies abroad.

Hence, in 2012 Jan enrolled in the "Research Master Nutrition and Metabolism" at Maastricht University. As part of this training he assisted in the Cold Acclimation Study in the research group of Prof. van Marken-Lichtenbelt.

In the research group of Prof. Schrauwen and Prof. Hesselink, Jan started his master thesis project, which elaborated on the molecular role of SIRT1 in calorie-mimetic effects during resveratrol supplementation.

With the molecular insight and his practical experience that he had obtained at Maastricht University and previous research projects, Jan designed an innovative research project that aims to investigate a novel risk factor in metabolic diseases: circadian disturbances. His PhD project proposal with the working title "Fine-Tuning Treatment Timing in Type 2 Diabetes Mellitus" was rewarded with the NUTRIM NWO Graduate Program Scholarship, in order to allow him to examine the role of the biological clock in disturbed energy metabolism. 